UNIVERSIDAD POLITÉCNICA DE MADRID

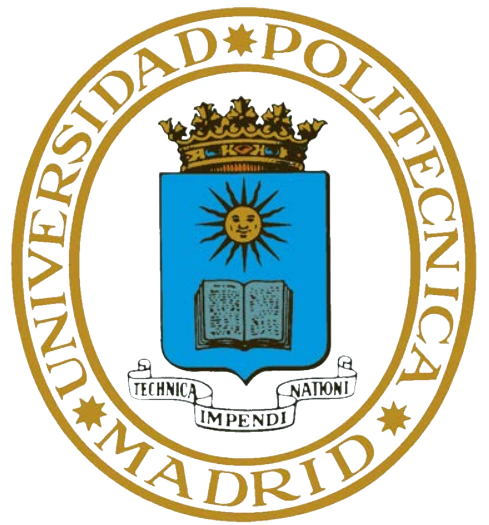

ESCUELA TÉCNICA SUPERIOR DE INGENIEROS INFORMÁTICOS

\title{
On the Security of Cache Algorithms
}

\author{
PH.D THESIS
}

Pablo Cañones Martín, M.R. 

DEPARTAMENTAMENTO DE LENGUAJES Y SISTEMAS INFORMÁTICOS E INGENIERIA DE SOFTWARE

ESCUELA TÉCNICA SUPERIOR DE INGENIEROS INFORMÁTICOS

\section{On the Security of Cache Algorithms}

SUBMITTED IN PARTIAL FULFILLMENT OF THE REQUIREMENTS FOR THE DEGREE OF:

Doctor of Philosophy in Software and Systems

Author: Pablo Cañones Martín, M.R.

Advisor: Dr. Boris Köpf

March 2019 
Copyright (C) 2019 by Pablo Cañones Martín, M.R. 


\section{Contents}

Abstract of the Thesis $\quad$ v

$\begin{array}{ll}\text { Resumen de la Tesis } & \text { vii }\end{array}$

$\begin{array}{ll}\text { Acknowledgments } & \text { ix }\end{array}$

List of Figures $\quad$ x

List of Tables $\quad$ xiii

List of Publications $\quad$ xiv

1 Introduction 1

2 Two Models of Cache Algorithms $\quad 8$

2.1 The Automata-based Model . . . . . . . . . . . . . . . 9

2.2 The Permutation-based Model . . . . . . . . . . . . . . . . 11

2.2.1 Modeling Fully Associative Caches . . . . . . . . . . . . . 11

2.2.2 Modeling Set-associative Caches . . . . . . . . . . . . . 14

2.3 Connecting Both Models . . . . . . . . . . . . . . . . 14

2.4 Caches as Mealy Machines . . . . . . . . . . . . . . . . . . 19

3 Security Analysis of Caches $\quad 21$

3.1 Information Theory Model . . . . . . . . . . . . . . . 21

3.1.1 Attack Scenario as a Markov Chain . . . . . . . . . . . 21

3.1.2 Observations as a Security Guarantee . . . . . . . . 22

3.2 Types of Attackers . . . . . . . . . . . . . . . 23

3.2.1 Time-based Attacker . . . . . . . . . . . . . . 24

3.2.2 Trace-based Attacker . . . . . . . . . . . . . . . . 24

3.2.3 Access-based Attacker . . . . . . . . . . . . 25

4 Information Absorption and Extraction $\quad 27$

4.1 Information Absorption . . . . . . . . . . . . . . . 28

4.2 Information Extraction . . . . . . . . . . . . . 29

4.2.1 Probing Strategies ... . . . . . . . . . 29 
4.2.2 An Algorithm for Information Extraction . . . . . . . . 32

5 Upper bounds on Leakage 36

5.1 Time and Trace-based Attackers . . . . . . . . . . . . . . 37

5.2 Access-based Attacker . . . . . . . . . . . . . . . . . . 40

5.2.1 Information Absorption . . . . . . . . . . . . . 40

5.2 .2 Information Extraction . . . . . . . . . . . 50

5.2 .3 Set-Associative Caches . . . . . . . . . . . 57

6 CacheAudit With Information Extraction 63

6.1 CacheAudit's Over Approximations . . . . . . . . . . . . . . . 64

6.2 Upper Bounds for AES . . . . . . . . . . . . . . . . 65

6.3 Upper Bounds for Sosemanuk and HC-128 . . . . . . . . . . . 69

7 Competitive Analysis $\quad 71$

7.1 Time-based Attacker . . . . . . . . . . . . . . 72

7.1 .1 Leak Ratio . . . . . . . . . . . . . . . . . . 73

7.1.2 Characterizing the Leak Ratio . . . . . . . . . . . 74

7.1 .3 Discussion .................. 77

7.1.4 Leak Ratio from a Pair of Traces . . . . . . . . . . 78

7.1.5 Approximation of the Leak Ratio from a Single Trace . . . 80

7.1.6 A Linear Lower Bound on the Leak Ratio . . . . . . . . . . 82

7.2 Access-based Attacker . . . . . . . . . . . . . . 86

8 Related Work $\quad 89$

9 Conclusions 92

9.1 Contributions ........................ 93

9.2 Future Work ........................... 95

$\begin{array}{ll}\text { Bibliography } & 96\end{array}$

$\begin{array}{lr}\text { Appendix } & 102\end{array}$ 


\section{ABSTRACT OF THE THESIS}

Cache memories are an important component of modern computers as they reduce the latency gap when retrieving data from main memory. However, they are also very susceptible to side channel attacks as an attacker who can measure the execution time of a program using the cache can obtain information about the memory access pattern of the program.

In this thesis we address the security aspects of the cache, specifically the cache algorithm; the logical component that organizes memory stored the cache and decides which parts of the cache to empty when new data has to be stored. We aim to obtain results that give an idea of how secure current cache algorithms are against side channel attacks and if there is a cache algorithm (or a class of them) that is predominantly better in terms of security. We propose two measures of security aimed at different aspects of the security of cache algorithms under three common types of cache attackers, time, trace and accessbased attackers, and obtain results on several cache algorithms.

Upper bounds on the security of cache algorithms are obtained by focusing on the execution where the cache algorithm leaks the most information. This analysis provides security guaranties that are independent of the execution and therefore gives information only about the cache algorithm. However, the use of upper bounds is not suited for comparing cache algorithms as it does not guaranty that both algorithms are fairly compared. We obtain upper bounds on the leakage for the three types of attackers and different classes of cache algorithms. An interesting property of all these results is that they are independent of the actual data a program uses, they only depend on the amount of memory that has to be stored in the cache.

For the time and trace-based attackers we obtain upper bounds for the leakage for a large class of cache algorithms, automata-based cache algorithms. Incidentally we show that, under this analysis and for the time and trace-based attackers, all cache algorithms leak the same amount of information.

For the access-based attacker we propose a new model that better captures how this attacker retrieves information from the cache. The access-based attacker can not see the actual content of the cache but has to probe it, that is, 
has to access data and check whether or not it is stored in the cache, thus modifying the configuration of the cache. Then, the security of the cache against an access-based attacker is characterized by two aspects: (1) the amount of information about the victim that can be absorbed by the cache and (2) the amount of information that can effectively be extracted from the cache by the attacker. We define these two notions and propose an algorithm to compute the information extraction for any cache algorithm.

Using this model for the access-based attacker we study an smaller class of cache algorithms, permutation-based cache algorithms. For those algorithms, we obtain upper bounds on the leakage that vary depending on the cache algorithm in question, contrary to what happened for the time and trace-based attacker. When considering set-associative caches we show that the intuitive idea that several cache sets should reduce the leakage is not necessarily true.

Additionally, we apply our algorithm of information extraction to improve the security bounds of the CacheAudit static analyzer for the case of the accessbased attacker and analyze the security of several cryptographic algorithms.

Competitive analysis compares the leakage of two cache algorithms on every possible program. This allows to quantify how different two cache algorithms are in terms of security. However, competitive analysis always requires to compare one cache algorithm with respect to another and it is not suited to analyze the security of cache algorithms individually.

For the time-based attacker we show that the leak competitiveness relationship is symmetric in the cache algorithms for every pair of automata-based cache algorithms. This implies that no cache algorithm dominates another in terms of timing leakage, which is surprising since dominance results do exist in the case of performance. Moreover, when restricted to cache algorithms with finite control (common in hardware-based implementations) the leak competitiveness relationship between two cache algorithms is either asymptotically linear or constant, no other shapes are possible.

For the access-based attacker, we show examples of cache algorithms (LRU, FIFO and PLRU) that show that, for this attacker, dominance relationships do exist, in contrast to the time-based attacker. 


\section{RESUMEN DE LA TESIS}

Las memorias cachés son una componente importante de los ordenadores modernos ya que reducen la latencia al obtener datos de la memoria principal. Sin embargo, también son muy susceptibles a los ataques de canal lateral dado que un atacante que pueda medir el tiempo de ejecución de un programa que utiliza la caché puede obtener información acerca del patrón de acceso del programa a la memoria.

En esta tesis nos centramos en los aspectos de seguridad de la caché, concretamente en el algoritmo de caché; la componente lógica que organiza la memoria almacenada en la caché y decide qué partes de la caché hay que vaciar cuando se debe almacenar nueva información. Nuestro objetivo es obtener resultados que den una idea de cómo de seguros son los algoritmos de caché actuales frente a los ataques de canal lateral y si existe un algoritmo de caché (o una clase de ellos) que sea predominantemente mejor en términos de seguridad. Proponemos dos medidas de seguridad orientadas a diferentes aspectos de la seguridad de los algoritmos de caché frente a tres tipos comunes de atacantes de caché, tiempo, traza y acceso, y obtenemos resultados sobre diversos algoritmos de caché.

Las cotas superiores de seguridad de los algoritmos de caché se obtienen al centrarse en la ejecución en la que el algoritmo de caché filtra la mayor cantidad de información. Este análisis ofrece garantías de seguridad que son independientes de la ejecuición y por tanto da información sólo sobre el algoritmo de caché. Sin embargo, el uso de cotas superiores no es apropiado para comparar algoritmos de caché ya que no se puede garantizar que ambos algoritmos sean comparados en igualdad de condiciones. Obtenemos cotas superiores de la filtración para los tres tipos de atacantes y diferentes clases de algoritmos de caché. Una propiedad interesante de estos resultados es que son independientes de la porción concreta de memoria que usa un programa, únicamente dependen de la cantidad de memoria que debe ser almacenada en la caché.

Para los atacantes de tiempo y traza obtenemos cotas superiores de filtración para una clase muy amplia de algoritmos de caché, los algoritmos basados en autómata. Incidentalmente demostramos que, para este análisis y para los atacantes de tiempo y traza, todos los algoritmos de caché filtran la misma cantidad de información. 
Para el atacante de acceso proponemos un nuevo modelo que captura mejor cómo este atacante obtiene información de la caché. El atacante de acceso no puede ver el contenido de la caché y debe sondarla, esto es, debe acceder partes de la memoria y comprobar si están o no almacenadas en la caché, con lo que modifica la configuración de la caché. Entonces, la seguridad de la caché frente al atacante de acceso se caracteriza por dos aspectos: (1) la cantidad de información sobre la víctima que puede ser absorbida por la caché y (2) la cantidad de información que puede ser en efectivamente extraída de la caché por el atacante. Definimos estas dos nociones y proponemos un algoritmo que calcula la extracción de información para cualquier algoritmo de caché.

Utilizando este modelo para el atacante de acceso estudiamos una clase más pequeña de algoritmos de caché, los algoritmos de caché basados en permutaciones. Para estos algoritmos, obtenemos cotas superiores de filtración que varían dependiendo del algoritmo de caché en cuestión, contrariamente a lo que pasaba para los atacantes de tiempo y traza. Si además consideramos cachés con varios conjuntos independientes, comprobamos que la idea intuitiva de que varios conjuntos de caché deberían reducir la filtración no es necesariamente cierta.

Aplicando nuestro algoritmo de extracción de información, mejoramos las cotas de seguridad del analizador estático CacheAudit para el atacante de acceso y analizamos la seguridad de diversos algoritmos criptográficos.

El análisis de competitividad compara la filtración de dos algoritmos de caché para cualquier programa posible. Esto permite cuantificar cómo de diferentes dos algoritmos de caché son en términos de seguridad. Sin embargo, el análisis de competitividad siempre requiere comparar un algoritmo de caché con otro y no es apropiado para analizar individualmente la seguridad de los algoritmos de caché.

Para el atacante de tiempo demostramos que la relación de competitividad para filtración es simétrica en los algoritmos de caché para cualquier pareja de algoritmos de caché basados en autómata. Esto implica que ningún algoritmo de caché domina a otro en términos de filtración de tiempo, lo cual es sorprendente dado que sí existen resultados de dominación en el caso de la eficiencia. Además, si nos restringimos a algoritmos de caché con control finito (comunes en implementaciones basadas en hardware) la relación de competitividad para filtración entre dos algoritmos de caché es o bien asintóticamente lineal o bien constante, no son posibles otros comportamientos.

Para el atacante de acceso mostramos ejemplos de algoritmos de caché (concretamente LRU, FIFO y PLRU) que muestran que, para este atacante, sí existen relaciones de dominación, contrastando con el atacante de tiempo. 


\section{ACKNOWLEDGMENTS}

Thanks to Boris for the advice, my family for the support and Pepe for the company during all these years. 


\section{List of Figures}

2.1 An example of two consecutive cache hits with PLRU [DKMR15]. 13

4.1 Example of a Mealy machine. . . . . . . . . . . . . 28

5.1 Information absorption of a 4-way cache set. Figure 5.1a depicts the case of a filled initial cache, part 5.1b an empty one. In both figures, the horizontal axis depicts the footprint, i.e., the number of memory blocks used. The vertical axis depicts the absorbed information on a logarithmic scale, that is, converting the number observations into bits of information. Note that in Figure $5.1 \mathrm{~b}$, the line for LRU and FIFO coincides. . . . . . . . . 50

5.2 Information extraction of different replacement policies on a 4way cache set. Figures 5.2a, 5.2c and 5.2e depict the case of a filled initial cache, $5.2 \mathrm{~b}, 5.2 \mathrm{~d}$ and $5.2 \mathrm{f}$ an empty one. In all figures, the horizontal axis depicts the footprint, i.e., the number of memory blocks used. The vertical axis depicts the extracted information on a logarithmic scale, that is, converting the number observations into bits of information. . . . . . . . . . 56

5.3 Information absorption of different replacement policies on a 4way cache with 20 memory blocks of footprint and an increasing number of cache sets. Figure 5.3a depicts the case of a filled initial cache, part $5.3 \mathrm{~b}$ an empty one. In all figures, the horizontal axis depicts the number of cache sets. The vertical axis depicts the extracted information on a logarithmic scale, that is, converting the number observations into bits of information. Note that in Figure 5.3b, the line for LRU and FIFO coincides. . . . . . 58

5.4 Information extraction of different replacement policies on a 4way cache with 20 memory blocks of footprint and an increasing number of cache sets. Figures 5.2a, 5.2c and 5.2e depict the case of a filled initial cache, $5.2 \mathrm{~b}, 5.2 \mathrm{~d}$ and $5.2 \mathrm{f}$ an empty one. In all figures, the horizontal axis depicts the number of cache sets. The vertical axis depicts the extracted information on a logarithmic scale, that is, converting the number observations into bits of information 
5.5 Difference in information absorption and extraction between one cache set and as many cache sets as memory blocks. Here we consider a 4-way cache starting on an empty configuration. Solid lines represent the leakage of one single cache set that stores all the memory blocks (one line per cache algorithm) and the dashed line represents the absorption of as many cache sets as memory blocks, i.e. one block per cache set (the same line for all algorithms). In all figures, the horizontal axis depicts the footprint. The vertical axis depicts the leakage on a logarithmic scale, that is, converting the number observations into bits of information. Note that in Figures 5.5a and 5.5c, the line for LRU and FIFO coincides. For small footprints, several cache sets produce less or equal leakage than one cache set but, when the footprint is large enough, having all the memory blocks in one cache set produces less leakage than having each one alone in a cache set. . . . . . . . . . . . . . . . . 62

6.1 Information absorption and extraction (in bits) for the AES-256 execution on a 4-way cache, for filled and empty initial cache states. Figures $6.1 \mathrm{a}, 6.1 \mathrm{c}$ and $6.1 \mathrm{e}$ depict the case of a filled initial cache, $6.1 \mathrm{~b}, 6.1 \mathrm{~d}$ and $6.1 \mathrm{f}$ an empty one. The horizontal axis depicts the size of the cache in $\mathrm{KB}$, the vertical axis depicts the extracted information in logarithmic scale, that is, converting the number observations into bits of information. Points represent the upper bounds on the leakage, assuming an even distribution of the memory blocks and considering the worst-case scenario. Lines represent the values computed by CacheAudit. . 66

6.2 Information absorption and extraction (in bits) for the Sosemanuk and HC-128 execution on a 4-way cache with line size of 64 bytes. Figures $6.2 \mathrm{a}, 6.2 \mathrm{c}$ and $6.2 \mathrm{e}$ depict the leakage of Sosemanuk, $6.2 \mathrm{~b}, 6.2 \mathrm{~d}$ and $6.2 \mathrm{f}$ depict the leakage of HC-128. The horizontal axis depicts the size of the cache in $\mathrm{KB}$, the vertical axis depicts the extracted information in logarithmic scale, that is, converting the number observations into bits of information. Points represent the upper bounds on the leakage, assuming an even distribution of the memory blocks and considering the worst-case scenario. Lines represent the values computed by

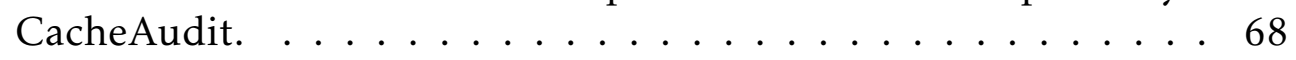


7.1 Example of the behavior of the leak ratios of the cache algorithms from Example 7.8. Figure 7.1a shows two leak ratios that grow asymptotically linearly and where the slopes can give a slight advantage of one algorithm over the other. Figure 7.1b shows two leak ratios that eventually become constant functions and were, for large lengths of traces, there is no advantage of one algorithm over the other. . . . . . . . . . . 76

A.1 Example of two sets of traces of memory blocks updating empty caches under cache algorithms LRU and FIFO. Each configuration of the cache shows the blocks cached between brackets, the symbol $\perp$ represents a line with no memory block cached in it and the bold face memory block is the next one to be evicted (the least recently used for LRU and the first to be cached for FIFO). Each update of the cache configuration is labeled with the accessed memory block (over the arrow) and the number of misses it produces on LRU and FIFO respectively (under the arrow). At the end of each trace we have the total number of misses the trace produces on LRU and FIFO respectively. . . . . . 102 


\section{List of Tables}

4.1 Partition from Example 4.6. . . . . . . . . . . . . 32 


\section{LIST OF PUBLICATIONS}

This thesis comprises two papers, the first has been published to a peer-reviewed academic conference. The second one is currently under review. The following list summarizes the aforementioned publications:

- Pablo Cañones, Boris Köpf and Jan Reineke. Security Analysis of Cache Replacement Policies

In Proceedings of $6^{\text {th }}$ ETAPS Conference on Principles of Security and Trust. 2017 [CKR17].

- Pablo Cañones, Boris Köpf and Jan Reineke.

On the Incomparability of Cache Algorithms in Terms of Timing Leakage

In Logical Methods in Computer Science [CKR19]. 


\section{CHAPTER 1}

\section{INTRODUCTION}

Nowadays, everyone desires that his or her computer runs as fast as possible. Computer manufacturers design hardware and write code with the goal of saving execution time in mind. This way, modern computers incorporate functionalities like speculative or out of order execution, branch predictors or cache memories to reduce to the minimum the time a processors' core is idle.

This work focuses on cache memory, one of the most important components of the memory hierarchy of a computer. Caches are small and fast memories located close to the cores inside a processor whose aim is to reduce the time a core has to wait to receive data from main memory (commonly referred to as the latency gap). Because of the technology used in caches and their proximity to the processor's core, retrieving data from the cache is significantly faster than from main memory. In some cases, "accessing cached data is up to two orders of magnitude faster than accessing main memory" [GBK11].

However, caches are very small in comparison to the main memory and so can only store a small fraction of the data. For this reason, caches tend to store the data a program is currently using and the cache logic, which in this work we refer to as the cache algorithm, is the one that decides which data is stored in the cache. We now explain the actual behavior of the cache and some of the terms we use in the thesis.

Caches store data as memory blocks of a fixed size, each block containing several adjacent memory addresses. When a program asks for an address in memory, which in terms of the cache is the block that contains it, the cache algorithm decides whether this particular block is currently cached. If the memory block is cached (a cache hit), it is served to the processor in a short period of time. If, on the other hand, the memory block is not cached (a cache miss), it has to be retrieved from main memory which takes longer. Apart from this, the newly retrieved memory block has to be stored in the cache for future uses. The cache algorithm selects another memory block to evict from 
the cache in order to make room for the new memory block.

Caches are a good improvement against the latency gap; not all memory accesses are going to be cache hits but, with the right cache algorithm controlling the cache, a good portion of them are hits which speeds up the computation. The search for a good cache algorithm, in the sense of reducing the latency gap, is a widely studied field with several approaches such as worst-case analysis [Gru12, Wil05], benchmark analysis [AZMM04, CH01], comparison analysis [Dor10, RG08, ST85] and the proposal of new cache algorithms [MM03, QJP ${ }^{+}$07].

But there is a big downside to caches and for basically every element of hardware or software that aims to improve execution time: the timing improvement is not uniform over all executions of a program but depends on the specific input the user gives to the program. For the case of caches, if a program uses some secret inputs (such as a cryptographic key or any other sensitive data) and the way the program accesses the memory (that goes through the cache) depends on these secret inputs, the behavior of the cache when providing these sensitive access to memory may leak information about the secret inputs. This constitutes a side channel through the cache that has been used to recover keys from implementations of cryptographic algorithms that use the cache (for example AES) [AK06, ASK07, Ber05, GBK11, LYG ${ }^{+}$15, OST06, YF14] but it also has been recently used to obtain information on the memory distribution of programs $\left[\mathrm{KGG}^{+} 18, \mathrm{LSG}^{+} 18, \mathrm{GRB}^{+} 17\right]$ or simply as a very efficient covert channel [MWS $\left.{ }^{+} 17\right]$.

This negative aspect of cache memory is the motivation for our work. There is no evident way of disabling this side channel, as disabling the cache would mean increasing the latency lag to a point where the computation would be severely affected. Several approaches aim to the best way to mitigate the leakage of information through the side channel. This motivates studies that aim to give security guarantees on either the cache or the program using sensitive information. In particular, we focus our attention on the cache algorithm. There are other aspects to the security of the cache that are covered by other authors such as the architecture or the distribution of data inside the cache [LWML16, HL17, ZL14].

One way of studying how side-channels affect the security is by defining a quantifiable measure of security related to the information an attacker may obtain from the side-channel. The theoretical scenario we use to establish this notion of security is the following: the victim runs a program, whose input and execution depend on some secret value, on a platform with cache memory. The execution of the program translates, in terms of the cache, into a trace of memory blocks the cache has to provide. An attacker uses the cache sidechannel to obtain an observation about the behavior of the cache on the trace of memory blocks the victim's program used. This observation does not hold information about the secret by itself but, if different secret inputs produce 
different observations, the attacker can discard some secret inputs that are inconsistent with the obtained observation. Using this scenario and notions of information theory, we establish security guarantees about the cache sidechannel based on the number of different observations the attacker may obtain given the set of possible traces of memory blocks the victim may use, that is, the leakage of the side-channel.

In the literature there exist three types of attacker depending on their capabilities of observing the cache [AK06]. (1) The time-based attacker observes the overall execution time of the program and deduces the number of memory accesses that were misses [ASK07, Ber05, BM06, TSS ${ }^{+} 03$ ]. (2) The tracebased attacker observes whether each individual memory access is a hit or a miss [AK06, BZB ${ }^{+} 05$, Pag02, ZW10]. (3) The access-based attacker obtains information about the final configuration of the cache after the victim finishes his computation [GBK11, OST06].

\section{Open questions}

Using this framework for analyzing the security of side channels, focusing our attention on caches and considering the three types of attackers we identify the following open problems:

- What's the worst a cache algorithm can do in terms of security? There is no clear way to asses the overall security of a cache algorithm or if a cache algorithm is too insecure to run programs with sensitive data. To the best of our knowledge, the closest result of this kind is [DKMR15]. However, in this work the security of the cache algorithm is measured with respect to a program, it does not provide results that only depend on the cache algorithm and that are independent of the program the victim runs.

- Can we decide if one cache algorithm is better than another? There is no clear way to compare fairly the security of two cache algorithms so that we can identify a best cache algorithm either within a class of cache algorithms or in a pair of cache algorithms. By solving the previous problem, we would have a way to analyze individually the security a cache algorithm. However, using individual analysis to compare the leakage of two cache algorithms may not give a good comparison as the cache algorithms may not be analyzed on equality of opportunities.

\section{Contributions}

Our contributions to tackle these questions are the following:

Upper Bounds on Security. To address the first open question, we derive upper bounds on the leakage of a cache algorithm. Our main contribution is 
obtaining upper bounds that are independent of the program that interacts with the cache but we also obtain program dependent bounds for the accessbased attacker by improving current results. This contribution is divided into three parts:

1. We propose a new model for the access-based attacker that captures more realistically how this attacker obtains information from the cache.

Previous models [DKMR15] of the access-based attacker considers that the attacker obtains information about the configuration of the cache left by the victim simply by looking at the cache. In reality the only way an access-based attacker can know if a memory block is cached or not is by accessing it and observing if the access produces a cache hit or a cache miss, we denote this process probing the cache. Probing the cache introduces an extra problem for the attacker since the cache algorithm modifies the cache to hold the accessed block thus destroying information about the configuration left by the victim and providing less accurate information to the attacker. Our new model extends that of [DKMR15] including the probing phase.

We address this point in Chapter 4.

2. We obtain program independent upper bounds for various cache algorithms. We abstract from individual programs by considering a worstcase scenario, i.e. a set of traces of memory blocks that contains any possible trace with the same memory blocks the actual program uses. Since our notion of security depends on the number of observations the attacker may obtain from the side channel given a set of possible traces of memory blocks the victim may use, the value of leakage that we obtain from the worst-case scenario is an upper bound on the leakage of any set of traces that uses those same memory blocks.

Worst-case scenario produces an upper bound on the leakage that depends on the set of memory blocks under use. This means that the upper bound is independent of individual programs but not of specific sets of memory blocks. However, we prove that, for the cache algorithms that we consider, two sets of memory blocks of the same size (i.e. same amount of memory blocks) produce the same upper bound on leakage, which means that the upper bounds we derive do not depend on the actual memory blocks we use but rather how many of them we use, a value that we refer to as the footprint.

We develop new upper bounds on the leakage of several cache algorithms under the three types of attackers. For the time and trace-based attackers we obtain upper bounds on leakage for a large class of cache algorithms (automata-based cache algorithms) and incidentally conclude that, for the 
time and trace-based attacker and for the worst-case scenario analysis, all cache algorithms present the same security guarantees. For the accessbased attacker, we obtain results on the leakage for three common cache algorithms: LRU (Least Recently Used), FIFO (First In First Out) and PLRU (Pseudo Least Recently Used). Moreover, we study how the distribution of memory blocks over independent cache sets affects security and show that, in some cases, having several cache sets, which intuitively should reduce the leakage, actually produces more leakage than a single cache set.

We address this point in Chapter 5.

3. We obtain better program dependent upper bounds by incorporating our new model for the access-based attacker into the implementation of the CacheAudit analyzer. CacheAudit [DKMR15] is an static analyzer for the security of cache algorithms (in particular FIFO, LRU and PLRU) that gives an upper bound on the number of possible observations any of the three attackers can obtain from a specific program. However, for the access-based attacker, it assumes that the attacker can see the cache directly rather than having to probe it. By including the probe step to the CacheAudit implementation, we significantly improve the security guarantees given by CacheAudit, leading to gains of up to 50 bits for AES 256. We show that the executions of AES highly resemble the worstcase one (where the program uses every possible trace of memory blocks) whereas for other cryptographic algorithms, Sosemanuk and HC-128, it significantly differs.

We address this point in Chapter 6.

Leak Competitiveness. To address the second open question, we propose a novel approach for comparing pairs of cache algorithms, called leak competitiveness, based on notions of competitiveness. The original notion of competitiveness is used to compare the performance of online algorithms running the same input, for examples on cache algorithms see [RG08, ST85].

The main difference between leak competitiveness and standard competitiveness is that competitiveness is defined on single traces whereas leak competitiveness has to be defined on sets of traces. This corresponds to the fact that information flow is a hyperproperty [CS10].

We show that, for the time-based attacker, leak competitiveness is symmetric for any pair of cache algorithms. This implies that no cache algorithm dominates another in terms of leakage. This opposes to the case of performance where such dominances of one algorithm over the other exist [RG08]. Moreover, if we focus only on caches with finite control, which is natural in real hardware-based implementations of the cache, the leak-competitiveness 
relationship between two cache algorithms is either asymptotically linear or constant. No other shapes are possible.

The proofs of these results are based on three intermediate steps that are of independent interest.

1. The first is to show that a pair of traces of memory blocks precisely characterizes the leak competitiveness relationship between any two cache algorithms.

2. The second step is to show that we can actually identify a single trace of memory accesses for which the difference in number of misses between both algorithms matches their leak competitiveness to within a factor of 2. This is surprising in the light that leakage is a hyperproperty, i.e., it requires sets of traces to express.

3. The third step is to define a congruence on the cache contents of algorithms with finite control - but potentially infinite data - and to show that the resulting quotient is finite. Our characterization of leak competitiveness follows from the observation that, if the trace that witnesses the leak competitiveness is large enough, it will visit multiple congruent cache states, i.e. contain a cycle in the quotient. We then use a pumping argument to obtain a linear lower bound on the leak competitiveness from this cycle.

For the access-based attacker, we take the worst-case results on information extraction developed in Chapter 5 for LRU, FIFO and PLRU and show that, for this case of attacker, there exist differences in leak-competitiveness between cache algorithms which lead to dominance results.

We address this point in Chapter 7.

\section{Structure of the Thesis}

The thesis is structured as follows. In Chapter 2 we introduce the models we use to represent the cache algorithms and explain the classes of cache designs they capture. In Chapter 3 we model the victim and attacker, the attack scenario and characterize our notion of security guarantees. Also we introduce the three types of cache side-channel attackers. In Chapter 4 we refine the access-based attacker by introducing the notions of information absorption and extraction and propose an algorithm for computing the leakage of any cache algorithm. In Chapter 5 we introduce the concept of worst-case analysis and obtain upper bounds on the security of different cache algorithms under different types of attackers. In Chapter 6 we apply our refinement of the accessbased attacker and our algorithm the static analyzer CacheAudit and show 
how the security guarantees are improved. In Chapter 7 we introduce the concept of competitiveness analysis and obtain results on the security of different cache algorithms under different types of attackers. 


\section{CHAPTER 2}

\section{TWO MODELS OF CACHE ALGORITHMS}

Caches are fast but small memories that store a subset of the main memory's contents to bridge the latency gap between the CPU and the main memory. To profit from spatial locality and to reduce management overhead, main memory is logically partitioned into a set of memory blocks $B$. Each memory block contains a handful of adjacent memory addresses and it is cached as a whole in a cache line of the same size.

When a program needs to access a memory address, the cache logic determines to which memory block the address belongs to. Then it determines whether the block is stored in the cache ("cache hit") or not ("cache miss"). In the case of a miss, the cache algorithm decides which memory block to evict and replace by a new one.

To reduce the time to decide whether a block is cached or not, most caches are divided into cache sets, each containing an equal number of cache lines, this number is called the associativity of the cache. Similarly, each memory block can only be cached in a subset of cache lines. Depending on how many cache sets there are and how the blocks are distributed over the lines, different cache architectures arise:

- If all memory blocks can be cached in all cache lines, we say the cache is fully associative.

- If each memory block can only be cached in one line, we say the cache is direct mapped.

- If each block can be cached in any of the lines of a single cache set, we say the cache is set-associative. 
- If each block can be cached in several lines but these lines do not belong to the same cache set, we say the cache is skewed associative [Sez93].

Within each cache set, the cache algorithm decides which line to evict in the case of a miss.

We also make a couple of assumptions on our model of the cache:

- We assume that the cache algorithm never evicts a memory block if there is still space left, that is, the cache algorithm always prioritizes on invalid lines over valid ones. This property is satisfied by most cache algorithms as it is desirable that the cache memory stores as much data as possible to speed up computations.

- We assume that neither the victim or the attacker are able to evict memory blocks except for when they access memory blocks that produce a miss. This rules out situations like when the cache lines are invalidated when switching programs but allows to consider more powerful attackers and thus obtain more general security guarantees.

In this chapter we present two different approaches for modeling cache algorithms: the automata-based model, that covers a wide range of cache architectures, and the permutation-based model, a model used in previous work only applicable to a subset of cache algorithms and architectures. We also show that, if a cache algorithm can be expressed in both the automata-based and the permutation-based model, one can translate from one model to the other. Finally we show that a cache algorithm can also be represented as a Mealy machine provided the set of memory blocks is finite.

\subsection{The Automata-based Model}

We present the automata-based model for caches and explain the types of cache architectures that it captures. The intuition behind this model is making a explicit distinction between the logical component of the cache algorithm that organizes cache lines and keeps track of the ones that have been previously accessed (the control states) and the actual memory blocks the cache lines hold (the content functions).

Definition 2.1 (Automata-based model). An automata-based cache algorithm is a tuple $\mathrm{P}=\left(S_{\mathrm{P}}, i_{\mathrm{P}}, \eta_{\mathrm{P}}, \operatorname{tr}_{\mathrm{P}}\right.$, evict $\left._{\mathrm{P}}, C_{\mathrm{P}}\right)$, which consists of the following components:

- The set of control states, $S_{\mathrm{P}}$.

- The initial control state, $i_{\mathrm{P}} \in S_{\mathrm{P}}$. 
- The capacity of the cache, $\eta_{\mathrm{P}} \in \mathbb{N}$.

- The transition function, $\operatorname{tr}_{\mathrm{P}}: S_{\mathrm{P}} \times\left\{j_{1}, \ldots, j_{\eta_{\mathrm{P}}}\right\} \rightarrow S_{\mathrm{P}}$, that, upon a hit to one of its $\eta_{\mathrm{P}}$ cache lines, determines the new control state of the cache.

- The evict function, evict $: S_{\mathrm{P}} \times B \rightarrow S_{\mathrm{P}} \times\left\{j_{1}, \ldots, j_{\eta_{\mathrm{p}}}\right\}$, that, upon a miss, determines the new control state of the cache and the cache line to evict.

- The set of content functions, $C_{\mathrm{P}} \ni c_{\mathrm{P}}, c_{\mathrm{P}}=\left\{j_{1}, \ldots, j_{\eta_{\mathrm{P}}}\right\} \rightarrow B \cup\{\perp\}$, that map each cache line to the memory block its holds, or $\perp$ if the line is invalid. Note that the content function is injective in the lines, that is, each memory block can only be cached in one line at a time.

In the following, we drop the index $\mathrm{P}$ when it is clear from the context.

During runtime a cache configuration consists of the cache's control state and of its current content. A cache configuration $g=(s, c) \in G_{\mathrm{P}}=S_{\mathrm{P}} \times C_{\mathrm{P}}$ is updated as follows upon a memory access:

$$
\operatorname{upd}_{\mathrm{P}}((s, c), b):= \begin{cases}\left(s^{\prime}, c\right) & \text { if } \exists j: c(j)=b \wedge s^{\prime}=\operatorname{tr}_{\mathrm{P}}(s, j), \\ \left(s^{\prime}, c[j \leftarrow b]\right) & \text { if } \forall k: c(k) \neq b \wedge\left(s^{\prime}, j\right)=\operatorname{evict}_{\mathrm{P}}(s, b) .\end{cases}
$$

Upon a hit, the transition function is used to obtain the new control state. Upon a miss, the accessed block replaces one of the cached blocks, determined by the evict function.

The above definition of cache algorithms aims to capture a wide range of cache architectures and algorithms. We now mention some of the properties of the model:

- The transition function does not depend on the accessed memory block but on the lines that hold the blocks, this captures the idea that caches behave independently of the blocks they store. The only component of the configuration that changes in the case of a hit is the control states, this captures the idea that the cache algorithm uses recent history of memory accesses to decide which memory blocks to evict in the case of a miss.

- The evict function does depend on the accessed block so that we can model set-associative and skewed associative caches, where each memory block is stored in a specific set of cache lines and the algorithm decides which line to evict depending on the accessed memory block.

- Since the control states do not have a predefined structure, they can encode a wide range of deterministic replacement policies. In the next sections we will see a special case of cache algorithms, the permutationbased cache algorithms, and we will show how this algorithms are characterized with the automata-based model. 
Notation: The update function is lifted to traces of memory blocks, $t \in B^{*}$, recursively as follows:

$$
\begin{aligned}
\operatorname{upd}_{\mathrm{P}}((s, c), \epsilon) & :=(s, c), \\
\operatorname{upd}_{\mathrm{P}}((s, c), b \circ t) & :=\operatorname{upd}_{\mathrm{P}}\left(u p d_{\mathrm{P}}((s, c), b), t\right) .
\end{aligned}
$$

The only observable behavior of the cache memory is whether a memory access produces a cache hit or a cache miss. We represent this with the effect function, eff : $G_{\mathrm{P}} \times B \rightarrow\{\mathrm{H}, \mathrm{M}\}$, that, given a memory block and a configuration, returns the symbol $\mathrm{H}$ if the block is cached (a cache hit) and the symbol $\mathrm{M}$ if the block is not cached (a cache miss).

$$
e f f((s, c), b)= \begin{cases}H & \text { if } \exists j: c(j)=b, \\ M & \text { if } \forall j: c(j) \neq b .\end{cases}
$$

We extend the effect function to traces of memory blocks under algorithm $\mathrm{P}$ with $e f f_{\mathrm{P}}^{*}$ by concatenating the output of each individual block on the updated configuration:

$$
e f f_{\mathrm{P}}^{*}(g, b \circ t)=e f f(g, b) \circ e f f_{\mathrm{P}}^{*}\left(u p d_{\mathrm{P}}(g, b), t\right),
$$

Each attacker uses the effect function in a different way to obtain the side channel observation. We explain each one in particular in Section 3.2.

\subsection{The Permutation-based Model}

One special case of cache algorithms are the ones called permutation-based cache algorithms [AR13]. This class of cache algorithms are usually modeled by having the memory blocks of each cache set mapped to an age so that they are ordered. In the case of a miss, the cached memory block with the highest age is evicted. In the case of a hit, the cache algorithm reorders the ages of the blocks following a permutation function.

In this Section we show how previous work has modeled this type of cache algorithms for fully associative caches and set-associative caches.

\subsubsection{Modeling Fully Associative Caches}

In previous work [CKR17, DKMR15], the permutation-based model is defined on a fully associative cache in the following way:

Definition 2.2 (Permutation-based Model). Given a fully associative cache, a permutation-based cache algorithm is a tuple $\mathrm{P}=\left(A_{\mathrm{P}}, \widehat{G}_{\mathrm{P}}, \widehat{i g}_{\mathrm{P}}, \widehat{u p d}_{\mathrm{P}}\right)$ with the following components: 
- The associativity of the cache, $A_{\mathrm{P}}$, that is, the number of memory blocks that can be cached at the same time. In this model, the associativity coincides with the capacity $\eta_{\mathrm{P}}$

- A set of configurations, $\widehat{G}_{\mathrm{p}}$. Each configuration, $\hat{g} \in \widehat{G}_{\mathrm{p}}$, is a map from the set of memory blocks to a set of $A$ ages:

$$
\left.\hat{g} \in\left\{B \rightarrow\{0, \ldots, A\} \mid \forall b, b^{\prime} \in B: b \neq b^{\prime} \Rightarrow \hat{g}(b) \neq \hat{g}\left(b^{\prime}\right) \vee \hat{g}(b)=\hat{g}\left(b^{\prime}\right)=A\right)\right\} .
$$

Here, the youngest block has age 0 and the oldest cached block has age $A-1$. Age $A$ means that a block is not cached; it is the only age that can be shared by multiple blocks. The ages capture the order on which the blocks are evicted by new memory blocks.

- An initial configuration, $\widehat{i g}_{\mathrm{p}}$.

- An update function, $\widehat{u p d}_{\mathrm{P}}: \widehat{G}_{\mathrm{P}} \times B \rightarrow \widehat{G}_{\mathrm{p}}$ that, for every memory access, determines the new configuration.

In the following, we drop the index $\mathrm{P}$ when it is clear from the context.

The update function is defined as follows:

$$
\widehat{u p d}_{\mathrm{p}}(\hat{g}, b)\left(b^{\prime}\right)= \begin{cases}\Pi_{\mathrm{P}}\left(\hat{g}(b), \hat{g}\left(b^{\prime}\right)\right) & \text { if } \hat{g}\left(b^{\prime}\right)<A \wedge \hat{g}(b)<A, \\ 0 & \text { if } b^{\prime}=b \wedge \hat{g}(b)=A, \\ \hat{g}\left(b^{\prime}\right)+1 & \text { if } b^{\prime} \neq b \wedge \hat{g}\left(b^{\prime}\right)<A \wedge \hat{g}(b)=A, \\ \hat{g}\left(b^{\prime}\right) & \text { if } b^{\prime} \neq b \wedge \hat{g}\left(b^{\prime}\right)=A .\end{cases}
$$

- Upon a hit, the mapping from blocks to ages is rearranged to modify the order of eviction. The rearrangement of ages is determined by a permutation function $\Pi_{\mathrm{P}}\left(a, a^{\prime}\right):\{0, \ldots, A\}^{2} \rightarrow\{0, \ldots, A\}$; it modifies the current age $a^{\prime}$ of a block according to a base age $a$. This corresponds to the first case of (2.4).

- Upon a miss, the memory block with the oldest age is evicted, all the blocks increase their ages by one and the new block is mapped to age zero. This corresponds to the second and third case of (2.4).

- Independently of a hit or a miss, the blocks not cached (except for the accessed one in the case of a miss) remain mapped to age $A$. This corresponds to the fourth case of (2.4).

We define the effect function for configurations, $\widehat{e f f}: \widehat{G}_{\mathrm{P}} \times B \rightarrow\{\mathrm{H}, \mathrm{M}\}$, similarly to the automata-based model:

$$
\widehat{e f f}(\hat{g}, b)= \begin{cases}H & \text { if } \hat{g}(b)<A, \\ M & \text { if } \hat{g}(b)=A .\end{cases}
$$




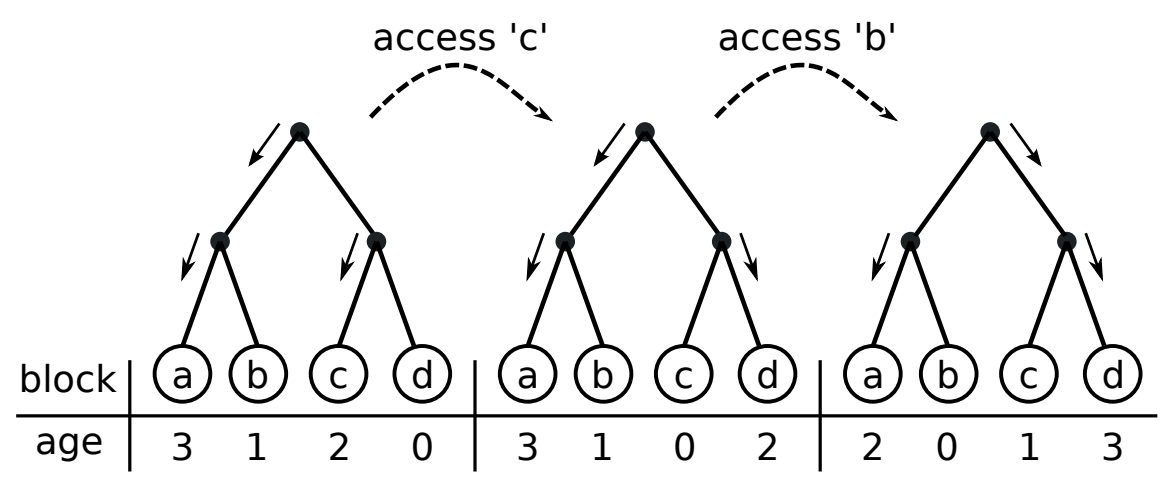

Figure 2.1: An example of two consecutive cache hits with PLRU [DKMR15].

We extend the effect function to traces of memory blocks under algorithm $\mathrm{P}$ with $\widehat{\text { eff }}_{\mathrm{P}}^{*}$ by concatenating the output of each individual block on the updated configuration:

$$
\widehat{e f f}_{\mathrm{P}}^{*}(\hat{g}, b \circ t)=\widehat{e f f}(\hat{g}, b) \circ \widehat{e f f}_{\mathrm{P}}^{*}\left(\widehat{u p d}_{\mathrm{P}}(\hat{g}, b), t\right),
$$

We introduce the definition of three common permutation functions following the model developed in [AR13].

The FIFO (First In First Out) replacement policy does not change the ages of the blocks upon cache hits. It is is thus modeled as the identity permutation.

$$
\Pi_{\mathrm{FIFO}}\left(a, a^{\prime}\right)=a^{\prime}
$$

The LRU (Least Recently Used) replacement policy sets the age of an accessed block to 0 upon a cache hit, making sure that the least-recently-used blocks get evicted upon misses. Formally, we cast this behavior as

$$
\Pi_{\mathrm{LRU}}\left(a, a^{\prime}\right)= \begin{cases}0 & \text { if } a^{\prime}=a \\ a^{\prime}+1 & \text { if } a^{\prime}<a \\ a^{\prime} & \text { if } a^{\prime}>a\end{cases}
$$

The PLRU (Pseudo Least Recently Used) is similar to LRU, but with a more complex permutation function. For an associativity which is a power of two, PLRU represents each cache set as a full binary tree storing the blocks at its leaves, and each non-leaf stores a bit which represents an arrow pointing to one of the children. Upon a cache miss, the block to be evicted is determined by following the arrows starting from the root. Upon any cache access (regardless whether it is a hit or a miss), the arrows on the way to the accessed block are flipped. Figure 2.1 shows an example of two consecutive cache hits in a 4-way cache. In this paper we assume that the associativity is always a power of two.

We formally define this PLRU permutation policy $\Pi_{P L R U}$ as 


$$
\Pi_{\mathrm{PLRU}}\left(a, a^{\prime}\right)= \begin{cases}0 & \text { if } a^{\prime}=a \\ a^{\prime} & \text { if } a \text { even } \wedge a^{\prime} \text { odd } \\ a^{\prime}+1 & \text { if } a \text { odd } \wedge a^{\prime} \text { even } \\ 2 \cdot \Pi_{\mathrm{PLRU}}\left(\lfloor a / 2\rfloor,\left\lfloor a^{\prime} / 2\right\rfloor\right) & \text { otherwise }\end{cases}
$$

\subsubsection{Modeling Set-associative Caches}

The previous model is only applicable to fully associative, where only the block mapped to the oldest age is evicted, independently of the accessed memory block. In the case of a set-associative cache, the lines are distributed in $m$ cache sets of equal associativity and each cache set stores a subset of the set of memory blocks $B$. This is done with a set function that maps each block to a cache set, set $: B \rightarrow\{1, \ldots, m\}$. We partition the set of memory blocks into disjoint subsets, each belonging to one cache set, $B=B^{1} \uplus \cdots \uplus B^{m}$.

In a set-associative cache of $m$ cache sets, each configuration is made of a set of $m$ maps from blocks to ages, $\left\{\hat{g}^{1}, \ldots, \hat{g}^{m}\right\}$, such that, for every $i \leq m$, the mapping $\hat{g}^{i}$ on the set of memory blocks $B^{i}$ behaves as a fully associative cache, see (2.3).

We define an extension of the update function to sets of maps by $\widehat{u p d}_{\mathrm{P}}^{\dagger}$ so that only the set that holds the accessed memory block is modified:

$$
\widehat{u p d}_{\mathrm{P}}^{\dagger}\left(\left\{\hat{g}^{1}, \ldots, \hat{g}^{m}\right\}, b\right)=\left\{\hat{g}^{1}, \ldots, \widehat{u p d}_{\mathrm{P}}\left(\hat{g}^{\text {set }(b)}, b\right), \ldots, \hat{g}^{m}\right\} .
$$

where $\widehat{u p d}_{\mathrm{p}}$ is the function defined in (2.4).

\subsection{Connecting Both Models}

We now show how to construct the automata-based model of a cache algorithm given its permutation-based model and prove that both models of the cache algorithm behave in the same way for any trace of memory blocks.

A configuration in the permutation-based model captures two things about the cache: the memory blocks currently cached (the ones mapped to ages bellow $A$ ) and the order on which they are evicted by new memory blocks (the ages of the cached blocks from older to younger). In the automata-based model, the memory blocks cached is captured by the content function. Then we define the control states of the algorithm to represent the order on which the memory blocks are evicted.

Fully associative caches Consider first a fully associative algorithm $\mathrm{P}$ given on the permutation-based model $\mathrm{P}=(A, \widehat{G}, \widehat{\hat{i g}}, \widehat{u p d})$. We define the analogous automata-based model, $\mathrm{P}=(S, i, \eta$, tr, evict,$C)$, with the following components: 
- The capacity is equal to the associativity $\eta=A$.

- The set of control states $S$ is a set of maps from cache lines to ages:

$$
S=\left\{s \in\left\{j_{1}, \ldots, j_{\eta}\right\} \rightarrow\{0, \ldots, \eta-1\} \mid \forall j, j^{\prime} \in\left\{j_{1}, \ldots, j_{\eta}\right\}: j \neq j^{\prime} \Rightarrow s(j) \neq s\left(j^{\prime}\right)\right\} .
$$

- The initial state is $i=\lambda j . j$.

- The transition function modifies the mapping of lines following the permutation function:

$$
\operatorname{tr}(s, j)\left(j^{\prime}\right)=\Pi\left(s(j), s\left(j^{\prime}\right)\right) .
$$

- The evict function evicts the line with the oldest age and modifies the mapping of lines to put the evicted line in the youngest age.

$$
\operatorname{evict}(s, b)=\left(j, s^{\prime}\right),
$$

where, $j$ is the line mapped to the oldest age, $s(j)=A-1$, and

$$
s^{\prime}\left(j^{\prime}\right)= \begin{cases}0 & \text { if } j^{\prime}=j, \\ s\left(j^{\prime}\right)+1 & \text { if } j^{\prime} \neq j .\end{cases}
$$

- The set of content functions is defined in the usual way, $C=\left\{j_{1}, \ldots, j_{\eta}\right\} \rightarrow$ $B \cup\{\perp\}$.

Set-associative caches We extend the previous automata-based model of fully associative caches to set-associative caches by considering that the lines in the content functions are divided into $m$ subsets of $A$ lines each,

$$
C=\left\{j_{1,1}, \ldots, j_{A, 1}, \ldots, j_{1, m}, \ldots, j_{A m}\right\} \rightarrow B \cup\{\perp\},
$$

and that the control states are a set of maps from lines to ages, $s=\left\{s^{1}, \ldots, s^{m}\right\}$, where, for every $i \leq m$, the mapping $s^{i}$ on the set of cache lines, $\left\{j_{1, i}, \ldots, j_{A, i}\right\}$ behaves as a fully associative cache, see (2.8).

We define the update function on set-associative caches, $u p d^{\dagger}$ as a function that only modifies the lines determined by the function set.

$$
u p d^{\dagger}((s, c), b):= \begin{cases}\left(s^{\prime}, c\right) & \text { if } \exists j_{k, i}: c\left(j_{k, i}\right)=b \wedge s^{\prime}=\operatorname{tr}^{\dagger}\left(s, j_{k, i}\right), \\ \left(s^{\prime}, c\left[j_{k, s e t(b)} \leftarrow b\right]\right) & \text { if } \forall j: c(j) \neq b \wedge\left(s^{\prime}, j_{k, s e t(b)}\right)=\operatorname{evict}^{\dagger}(s, b),\end{cases}
$$

where

$$
\begin{gathered}
\operatorname{tr}^{\dagger}\left(s, j_{k, i}\right)=\left\{s^{1}, \ldots, \operatorname{tr}\left(s^{i}, j\right) \ldots, s^{m}\right\}, \\
\operatorname{evict}^{\dagger}(s, b)=\left(\left\{s^{1}, \ldots, \operatorname{evict}\left(s^{\operatorname{set}(b)}, b\right), \ldots, s^{m}\right\}, j_{k, \text { set }(b)}\right) .
\end{gathered}
$$


and $t r$ and evict are defined as in (2.9) and (2.10) respectively.

We now show that indeed both models are equivalent in capturing the memory blocks cached and how the cached blocks change when accessing new memory blocks.

Lemma 2.3. Given a permutation-based cache algorithm $\mathrm{P}$, for every configuration in the automata-based model, $g \in G$, there exists a configuration in the permutation-based model, $\hat{g} \in \widehat{G}$, and for every configuration in the permutation-based model, $\hat{g} \in \widehat{G}$, there exists a configuration in the automata-based model, $g \in G$, such that, for any trace of memory blocks $t \in B^{*}$, every memory block $b$ is cached in $u p d(g, t)$ if and only if it is cached in $\widehat{u p d}(\hat{g}, t)$.

Proof. For illustration purposes, we focus first on a fully associative cache. We extend the proof to set-associative caches in the end of the proof.

Given a configuration in the automata-based model $g=(s, c)$, we construct the configuration $\hat{g}$ in the permutation-based model in the following way:

$$
\hat{g}(b)= \begin{cases}s(j) & \text { if } \exists j: c(j)=b, \\ A & \text { if } \forall j: c(j) \neq b .\end{cases}
$$

Note that, since $c$ is injective, if a memory block is cached there exists a single line mapped to it and so the configuration in the permutation-based model is well defined.

Given a configuration in the permutation-based model $\hat{g}$, we construct the configuration $g=(s, c)$ in the automata-based model in the following way:

$$
\begin{gathered}
c\left(j_{k}\right)= \begin{cases}b & \text { if } \exists b: \hat{g}(b)=k, \\
\perp & \text { if } \forall b: \hat{g}(b) \neq k .\end{cases} \\
s\left(j_{k}\right)=k .
\end{gathered}
$$

We show that, for every $i, \operatorname{upd}\left(g, b_{1} \ldots b_{i}\right)$ and $\widehat{u p d}\left(\hat{g}, b_{1} \ldots b_{i}\right)$ have the same memory blocks cached and not cached by induction on $i$.

Notation. We write $t=b_{1} \ldots b_{n}$, and denote, for every $i \leq n, g_{i}=u p d\left(g, b_{1} \ldots b_{i}\right)$ and $\hat{g}_{i}=\widehat{u p d}\left(\hat{g}, b_{1} \ldots b_{i}\right)$.

Induction base. For $i=0$ we have that $g_{0}=g$ and $\hat{g}_{0}=\hat{g}$. For any memory block $b$, it is cached in $g=(s, c)$, that is, there exists a line $j$ such that $c(j)=b$, if and only if it is cached in $\hat{g}$, that is, $\hat{g}(b)<A$. Conversely, $b$ is not cached in $g$, that is, for all lines $j, c(j) \neq b$, if and only if it is not cached in $\hat{g}$, that is, $\hat{g}(b)=A$. 
Induction step. We know that $g_{i}=u p d\left(g, b_{1} \ldots b_{i}\right)$ and $\hat{g}_{i}=\widehat{u p d}\left(\hat{g}, b_{1} \ldots b_{i}\right)$ have the same memory blocks cached and not cached and we check for $i+1$.

We update $g_{i}=\left(s_{i}, c_{i}\right)$ with memory block $b_{i+1},\left(s_{i+1}, c_{i+1}\right)=u p d\left(\left(s_{i}, c_{i}\right), b_{i+1}\right)$.

- In the case of a hit, let $j_{i+1}$ be the line such that $c_{i}\left(j_{i+1}\right)=b_{i+1}$. The content of the cache does not change, $c_{i+1}=c_{i}$.

- In the case of a miss, let $\tilde{j}$ be the line returned by the evict function, $\left(s_{i+1}, \tilde{j}\right)=\operatorname{evict}_{\mathrm{P}}\left(s_{i}, b_{i+1}\right)$. The content function is modified to cache $b_{i+1}$, that is, $c_{i+1}=c_{i}\left[\tilde{j} \leftarrow b_{i+1}\right]$.

The updated control state in both cases is:

$$
s_{i+1}(j)= \begin{cases}\Pi\left(s_{i}(j), s_{i}\left(j_{i+1}\right)\right) & \text { if } \exists j_{i+1}: c_{i}\left(j_{i+1}\right)=b_{i+1}, \\ 0 & \text { if } \forall j^{\prime}: c_{i}\left(j^{\prime}\right) \neq b_{i+1} \wedge j=\tilde{j}, \\ s_{i}(j)+1 & \text { if } \forall j^{\prime}: c_{i}\left(j^{\prime}\right) \neq b_{i+1} \wedge j \neq \tilde{j},\end{cases}
$$

We construct state $\hat{g}_{i+1}$ following (2.11):

$$
\hat{g}_{i+1}(b)= \begin{cases}\Pi\left(s_{i}(j), s_{i}\left(j_{i+1}\right)\right) & \text { if } \exists j_{i+1}: c_{i}\left(j_{i+1}\right)=b_{i+1} \wedge \exists j: c_{i+1}(j)=b, \\ 0 & \text { if } \forall j^{\prime}: c_{i}\left(j^{\prime}\right) \neq b_{i+1} \wedge j=\tilde{j} \wedge \exists j: c_{i+1}(j)=b, \\ s_{i}(j)+1 & \text { if } \forall j^{\prime}: c_{i}\left(j^{\prime}\right) \neq b_{i+1} \wedge j \neq \tilde{j} \wedge \exists j: c_{i+1}(j)=b, \\ A & \text { if } \forall j: c_{i+1}(j) \neq b .\end{cases}
$$

We translate each case to a condition that does not depend on the content function:

1. This case occurs in the case of a hit, $\exists j_{i+1}: c_{i}\left(j_{i+1}\right)=b_{i+1}$, which is equivalent to $\hat{g}_{i}\left(b_{i+1}\right)<A$; and when $b$ is cached, $\exists j: c_{i+1}(j)=b$. Since, for hits, the content function does not change, this last condition is equivalent to $\exists j: c_{i}(j)=b$ which is equivalent to $\hat{g}_{i}(b)<A$.

2. This case occurs in the case of a miss, $\forall j^{\prime}: c_{i}\left(j^{\prime}\right) \neq b_{i+1}$, which is equivalent to $\hat{g}_{i}\left(b_{i+1}\right)=A$; when $b$ is cached, $\exists ! j: c_{i+1}(j)=b^{1}$; and it is cached in the line that was evicted when $b_{i+1}$ was accessed, $j=\tilde{j}$. These last two conditions are equivalent to $b=b_{i+1}$.

3. This case occurs in the case of a miss, $\forall j^{\prime}: c_{i}\left(j^{\prime}\right) \neq b_{i+1}$, which is equivalent to $\hat{g}_{i}(b)=A$; when $b$ is cached, $\exists ! j: c_{i+1}(j)=b \Leftrightarrow \hat{g}_{i+1}(b)<A^{1}$; but it is cached in a line that was not evicted when $b_{i+1}$ was accessed, $j \neq \tilde{j} \Leftrightarrow b \neq b_{i+1}$.

\footnotetext{
${ }^{1}$ The line is unique since $c_{i+1}$ is injective.
} 
4. This case occurs when $b$ is not cached, which implies that $b \neq b_{i+1}$. The content function only changes for $j_{i+1}$ (if it does) so actually, not being cached after the access is equivalent to not being cached before and being different to the accessed block, i.e. $\forall j: c_{i+1}(j) \neq b \Leftrightarrow \forall j: c_{i}(j) \neq b \wedge b \neq$ $b_{i+1} \Leftrightarrow \hat{g}_{i}(b)=A \wedge b \neq b_{i+1}$.

Translating all the conditions we arrive at:

$$
\hat{g}_{i+1}(b)= \begin{cases}\Pi\left(s_{i}\left(j^{\prime}\right), s_{i}(j)\right) & \text { if } \hat{g}_{i}\left(b_{i+1}\right)<A \wedge \hat{g}_{i}(b)<A, \\ 0 & \text { if } \hat{g}_{i}(b)=A \wedge b=b_{i+1}, \\ s_{i}\left(j^{\prime}\right)+1 & \text { if } \hat{g}_{i}\left(b_{i+1}\right)=A \wedge \hat{g}_{i}(b)<A \wedge b \neq b_{i+1}, \\ A & \text { if } \hat{g}_{i}(b)=A \wedge b \neq b_{i+1} .\end{cases}
$$

Which coincides with the configuration $\hat{g}_{i+1}=\widehat{u p d}\left(\hat{g}_{i}, b_{i+1}\right)$.

Therefore, reasoning like in the induction base step, a every memory block is cached in $g_{i+1}$ if and only if it is cached in $\hat{g}_{i+1}$.

Set-associative version. In the case of a set-associative cache, configuration $\hat{g}=\left\{\hat{g}^{1}, \ldots, \hat{g}^{m}\right\}$ in the permutation-based model is constructed from configuration $g=\left(\left\{s^{1}, \ldots, s^{m}\right\}, c\right)$ in the automata-based model so that, for every $i \leq m$ and every $b \in B_{i}$ :

$$
\hat{g}^{i}(b)= \begin{cases}s\left(j_{k, i}\right) & \text { if } \exists k: c\left(j_{k, i}\right)=b . \\ A & \text { if } \forall k: c\left(j_{k, i}\right) \neq b .\end{cases}
$$

Conversely, configuration $g=\left(\left\{s^{1}, \ldots, s^{m}\right\}, c\right)$ in the automata-based model is constructed from configuration $\hat{g}=\left\{\hat{g}^{1}, \ldots, \hat{g}^{m}\right\}$ in the permutation-based model so that, for every $i \leq m$ and every $b \in B_{i}$ :

$$
\begin{gathered}
c\left(j_{k, i}\right)= \begin{cases}b & \text { if } \exists b: \hat{g}^{i}(b)=j_{i, k}, \\
\perp & \text { if } \forall b: \hat{g}^{i}(b) \neq j_{i, k},\end{cases} \\
g^{i}\left(j_{k, i}\right)=k .
\end{gathered}
$$

Configurations $g$ and $\hat{g}$ verify that a memory block is cached in $g$ if and only if it is cached in $\hat{g}$. Since the update function for set-associative caches adapts the update function from fully associative caches, the rest of the proof is similar to the fully associative cache.

We use the previous Lemma to show that, for a permutation-based cache algorithm, any trace of memory blocks produces the same effect in both cache algorithm models. 
Proposition 2.4. Given a permutation-based cache algorithm $\mathrm{P}$, for every configuration $g=(s, c) \in G$ in the automata-based model there exists a configuration $\hat{g} \in \widehat{G}$ in the permutation-based model such that, for any trace of memory blocks $t \in B^{*}$,

$$
e f f^{*}(g, t)=\widehat{e f f}^{*}(\hat{g}, t) .
$$

Conversely, for every configuration $\hat{g} \in \widehat{G}$ in the permutation-based model there exists a configuration $g=(s, c) \in G$ in the automata-based model such that, for any trace of memory blocks $t \in B^{*}$,

$$
\widehat{e f f}^{*}(\hat{g}, t)=e f f^{*}(g, t) .
$$

Proof. Lemma 2.3 gives the construction of $\hat{g}$ given $g$ and vice versa. It also shows that, for every trace of memory blocks $t \in B^{*}$, the updated configurations $\operatorname{upd}(g, t)$ and $\widehat{u p d}(\hat{g}, t)$ have the same memory blocks cached and not cached.

Therefore, using the configurations given by the Lemma, the effect functions produce the same trace of hits and misses on both configurations.

\subsection{Caches as Mealy Machines}

Our two models of cache algorithms resemble very much the model of a Mealy machine, that is, an automata that, upon receiving an input symbol, a memory block, produces an output symbol, hit or miss, and transitions to a different state, a cache configuration. The main difference between our models and that of a Mealy machine is the fact that a Mealy machine considers a finite set of states whereas we assume that the number of cache configurations is infinite. However, if we restrict ourselves to a finite set of memory blocks, for any cache algorithm $\mathrm{P}$ there exists a finite set of configurations. Then, with a finite set of memory blocks a cache algorithm $\mathrm{P}$ is actually a Mealy Machine, that is, a six-tuple, $M_{\mathrm{P}}=(\Gamma, \Sigma, O, i \gamma, u p, o b s)$, with:

- A finite set of states, $\Gamma$ (the set of configurations, $G_{\mathrm{P}}$ ).

- A finite set of input symbols, $\sum$ (the set of memory blocks, $B$ ).

- A finite set of output symbols, $O$ (the effects of the memory blocks on the cache, $\{H, M\})$.

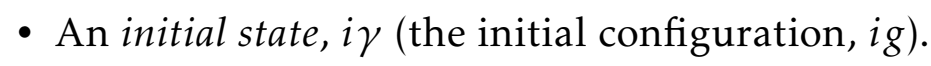

- A transition function up $: \Gamma \times \Sigma \rightarrow \Gamma$ (the update function, $u_{p} d_{\mathrm{p}}$ ).

- An output function obs : $\Gamma \times \Sigma \rightarrow O$ (the effect function, eff). 
The Mealy machine is defined similarly using the permutation-based model.

In Chapter 4 we formalize the model of the access-based attacker in terms of the memory blocks the attacker inputs and the effect they produce on the cache. Since these notions are common to all Mealy machines, we use their terminology to emphasize the generality of our results. 


\section{CHAPTER 3}

\section{SECURITY ANALYSIS OF CACHES}

In the previous chapter we saw two ways of modeling caches. Since our objective is to study their security against side channel attacks, we next need a way to model those attacks. To do so we take notions of information theory and model the side channel attack as a process in which the attacker reduces the uncertainty about the secret chosen by the victim by obtaining an observation about the behavior of the cache.

After the information theory model is established, we introduce the three types of attackers encountered in the literature and show how they obtain observations about the behavior of the cache.

\subsection{Information Theory Model}

In this section we characterize the security of the side channel using notions of information theory. This characterization is obtained by modeling the victim's secret as a random variable and considering how the probability of correctly guessing the secret varies before and after conditioning it to the observations of the side channel. We obtain a security guarantee of the side channel in terms of the number of different observations and attacker can obtain through the side channel.

\subsubsection{Attack Scenario as a Markov Chain}

Here we model the victim's choice of secret, the attackers observation, and the attacker's guess of the victim's secret as three random variables connected via a Markov chain. Using this Markov chain, we can obtain a bound on the probability of the attacker successfully guessing the victim's secret based on the size of the range of attacker's observations. 
We consider that the attack is in two phases:

- The victim chooses a secret $x$, like a cryptographic key, that he uses as input to a program. We model the choice of the secret with the random variable $X$. The victim runs a program that follows a specific execution path determined by the secret. In terms of the cache, the execution is a trace, $t$, of memory blocks from the set of possible traces, $T$, that make up the program, $t \in T \subseteq B^{*}$. The cache has to supply the memory blocks in trace $t$ or retrieve them from main memory.

- The attacker obtains an observation from the effect of the trace of memory blocks in the cache. Since the observation depends on the trace, and consequently on the secret, it is a random variable denoted by $Y$. Based on this observation, the attacker tries to guess the secret the victim used as input to the program. We model the guess of the secret in terms of the random variable $\hat{X}$. The attacker successfully guesses the secret when $X=\hat{X}$.

We also assume that all the information the attacker can obtain about the secret is captured in the observation of the side channel. This implies that we can express the three random variables as elements on a Markov chain:

$$
\underset{\text { Secret }}{X} \rightarrow \underset{\text { Observation }}{Y} \longrightarrow \underset{\text { Guess }}{\hat{X}}
$$

\subsubsection{Observations as a Security Guarantee}

Without considering the side channel, we have an upper bound on the probability of the attacker correctly guessing the secret in one attempt given by the probability of the most probable secret:

$$
P(X=\hat{X}) \leq \max _{x \in \operatorname{ran}(X)} P(X=x) .
$$

By modeling the attack as a Markov chain we obtain the following upper bound on the probability of successfully guessing the secret when considering the side channel observation:

Theorem 3.1 ([DKMR15]). Let $X \rightarrow Y \rightarrow \hat{X}$ be a Markov chain. Then:

$$
P(X=\hat{X}) \leq \max _{x \in \operatorname{ran}(X)} P(X=x) \cdot|\operatorname{ran}(Y)| .
$$

This bound is the one from (3.1) but increased by the size of the set of possible observations the attacker obtains from the side channel. This makes intuitive sense. If for all secrets and traces of memory blocks, the observation obtained from the side channel is the same, there is no increase on the 
probability of guessing the secret and the side channel leaks no information. Conversely, since we assume that the attacker has full knowledge of the implementation, which essentially means knowing the complete map from secrets to observations, if each secret produces a different observation, by knowing the observation the attacker knows the secret immediately.

This result bounds the probability of correctly guessing the secret in one attempt, which relates to the notion of min-entropy [Smi09]. Similar results can be obtained for Shanon-entropy [Smi09] and g-vulnerabilities [ACPS12a].

\subsection{Types of Attackers}

In this section we introduce the three most common attackers known in the literature of cache side channels. We explain how each attacker obtains the observations through the side-channel and introduce each attacker's observation function.

Throughout this section we always talk about an attacker that obtains the observation after the victim has accessed the cache and finished the computations on it, specially on the access-based attacker. This does not necessarily mean that the attacker only has one window to obtain observations. If the attacker can obtain observations at specific times during the execution, knowing precisely the current configuration of the cache and how many memory blocks the victim has accessed after the previous attacker's observation ${ }^{1}$, we can treat them as individual attacks, each one taking the current configuration of the cache as the configuration the victim starts computing with.

In practice, the attackers presented in this section require very strong assumptions on the architecture of the cache and the interaction of the victim and the attacker to obtain observations that only depend on the victim's actions. Real attacks tend to obtain noisy observations and so, obtain less information from the side channel than in the case of a noiseless observation. By focusing on very powerful attackers, we over approximate the security guarantees for real attacks but make sure to encompass all weaker but more realistic versions of them.

We assume that the attacker knows the program the victim is running, that is, the set of traces of memory blocks $T$ from where the victim selects one based on the secret, and the physical properties of the cache. This way, the attacker knows how many memory accesses the program does and the time it takes for a memory access depending on whether it is a hit or a miss, which allows him to distinguish if a memory access is a hit or a miss.

\footnotetext{
${ }^{1}$ This is commonly known as an synchronous attacker [OST06].
} 


\subsubsection{Time-based Attacker}

Time-based attacks are the most simple type of cache attacks. They are somewhat an evolution of the original timing attacks [KQQ99, $\mathrm{DKL}^{+}$98]. Original timing attacks exploit the fact that cryptographic applications have conditional branches that depend on the secret input and lead to different executions times that reveal information about the secret input.

The same idea is applied for programs that access different memory addresses depending on the secret input. If some input produces accesses to a set memory addresses that are close by, this translates into a few memory blocks to be stored in the cache which implies a lot of cache hits and a short execution time. If, on the other hand, the input produces accesses to a set of very dispersed memory addresses, this translates into a lot of different memory blocks to be stored in the cache, which leads to memory blocks being evicted, that implies more cache misses and a longer execution time. By being able to measure the execution time, an attacker can distinguish these two secret inputs [ASK07, Ber05, BM06, $\mathrm{TSS}^{+} 03$ ].

The observation the attacker obtains from the side channel is the number of misses the cache produces on the trace of memory blocks the victim uses. That is, the time observation function of algorithm $\mathrm{P}, \mathrm{P}_{\text {tme }}$, is defined as:

$$
P_{\text {tme }}: G \times B^{*} \rightarrow \mathbb{N} \cup\{0\} .
$$

The time observation function of algorithm $\mathrm{P}$ on a trace $t \in B^{*}$ starting in configuration $g$ is determined using the effect function as follows:

$$
\begin{aligned}
\mathrm{P}_{\text {tme }}(g, \epsilon) & :=0, \\
\mathrm{P}_{\text {tme }}(g, b \circ t) & :=(e f f(g, b)=\mathrm{M} ? 1: 0)+\mathrm{P}_{\text {tme }}\left(u p d_{\mathrm{P}}(g, b), t\right) .
\end{aligned}
$$

\subsubsection{Trace-based Attacker}

Trace-based attackers can see whether each individual access to the cache memory is a hit or a miss, rather than the sum of all of them as is the case of the time-based attacker. This is achieved by monitoring the power consumption of the cache $\left[\mathrm{BZB}^{+} 05\right]$. This provides a finer distinction between traces of memory blocks and a higher information leakage $\left[\mathrm{AK} 06, \mathrm{BZB}^{+} 05, \mathrm{Pag} 02, \mathrm{ZW} 10\right]$.

The observation the attacker obtains from the side channel is the trace of cache hits and misses that the cache produces on the trace of memory blocks the victim uses. That is, the trace-observation function of algorithm $\mathrm{P}, \mathrm{P}_{\mathrm{trc}}$, is defined as:

$$
P_{\text {trc }}: G \times B^{*} \rightarrow\{\mathrm{H}, \mathrm{M}\}^{*} .
$$

The trace observation function of algorithm $\mathrm{P}$ on a trace $t \in B^{*}$ starting in 
configuration $(g)$ is determined recursively as follows:

$$
\begin{aligned}
\left.\mathrm{P}_{\operatorname{trc}}(g), \epsilon\right) & :=\epsilon, \\
\left.\mathrm{P}_{\operatorname{trc}}(g), b \circ t\right) & :=e f f(b, g) \circ \mathrm{P}_{\operatorname{trc}}\left(u p d_{\mathrm{P}}(b, g), t\right),
\end{aligned}
$$

\subsubsection{Access-based Attacker}

While time-based and trace-based attackers observe the effect of the trace of memory blocks on the cache, whether memory accesses are hits or misses, access-based attackers obtain information about the final configuration of the cache after the trace of memory blocks has been input [GBK11, OST06]. This implies a distinction in the security analysis since the length of the traces does not solely influence the number of observations. More important is the number of different memory blocks the set of traces uses since, the larger the set of blocks, the more different configurations that can be reached and so a larger number of observations can be obtained.

In practice we make the distinction on what memory blocks the attacker can access and therefore distinguish inside the cache. Considering that the program the victim runs uses memory blocks from a finite set of victim's memory blocks, $B_{v} \subseteq B$, the set of attacker's memory blocks, $B_{a} \subseteq B$, can be of two types:

- The attacker can not access the victim's blocks, $B_{v} \cap B_{a}=\emptyset$, which is commonly known as a disjoint memory attacker.

- The attacker can access the victim's blocks $B_{v} \subseteq B_{a}$, which is commonly known as a shared memory attacker.

In both cases, we assume that the set of attacker's memory blocks is as big as the capacity of the cache algorithm, $\left|B_{a}\right|=\eta$. When obtaining upper bounds on the leakage for the access based attacker, Section 5.2, we will see how this benefits the attacker and allows to obtain sound upper bounds.

One basic theoretical approach to model disjoint and shared memory attackers is to consider that the attacker can observe the actual configuration of the cache after the victim has input the trace of memory blocks, that is, the exact control state and part of the content function depending on the type of memory access: either the attacker sees the mapping of lines to blocks including victim's ones (shared memory) or only sees which lines contain a victim's block but not which memory blocks in particular (disjoint memory). This is the attacker model considered in [DKMR15].

However, directly observing the state of the cache is not possible. The only way to know if a particular block is in the cache or not is by accessing it and observing whether that access is a hit or a miss. This presents an extra problem for the attacker since accessing new memory blocks modifies the cache and can 
eventually lead to a configuration that has no relation to the configuration left by the victim. We denote the process of accessing blocks to obtain information about the configuration of the cache probing the cache.

We model an access-based attack scenario to encompass the probing process in the following way:

1. The victim inputs any trace of memory blocks from a set $T \subseteq B_{v}^{*}$ on a cache memory starting from configuration $g_{v}$. This way, a set of traces produces a finite set of victim's configurations,

$$
G_{v}=\left\{g^{\prime} \in G_{\mathrm{P}} \mid \exists t \in T: \operatorname{upd}_{\mathrm{P}}\left(g_{v}, t\right)=g^{\prime}\right\}
$$

2. After the victim's computation, the attacker probes the cache by inputting a trace of memory blocks that modifies the configuration left by the victim and returns a trace of hits and misses.

Then, the access-observation function of algorithm $\mathrm{P}, \mathrm{P}_{\mathrm{acc}}$, is defined as:

$$
\mathrm{P}_{\mathrm{acc}}: G_{\mathrm{P}} \times B^{*} \rightarrow\{\mathrm{H}, \mathrm{M}\}^{*} \text {. }
$$

Observing a trace of hits and misses corresponds for the access-based attacker to observing a subset of victim's configurations that contains the actual configuration left by the victim. Using the trace of accessed memory blocks and the trace of hits and misses, the attacker restricts from the full set of victim's configurations to the ones that return the observed trace of hits and misses when the trace of memory blocks is accessed.

Note that, although the type of $P_{\text {trc }}$ and $P_{\text {acc }}$ is the same they do not represent the same observation. A trace-based attacker observes the effect of the trace used by the victim on the cache while an access-based attacker observes the effect of a trace he chooses on the configuration left after the victim's computation.

In the end, the security of the side channel for an access-based attacker is determined by the number of different observations the attacker can obtain but these observations depend on how many different configurations the executions of the victim's program may leave on the cache. Then we consider the security against an access-based attacker with two concepts: (1) how the traces of memory blocks used by the victim influence the configuration of the cache (i.e. the information the cache absorbs from the program) and (2) how accurately the attacker can distinguish these configurations by probing (i.e. how much information the attacker can extract from the cache).

We develop these two concepts in detail in Chapter 4 


\section{CHAPTER 4}

\section{INFORMATION ABSORPTION AND EXTRACTION}

As we have said before, previous work on analyzing the security of cache side channels under an access-based attacker assumes that the attacker can see the actual configuration of the cache [DKMR15]. In practice the attacker can not see the cache configuration and the only way to obtain information about the content of the cache is by accessing memory blocks and observing whether they produce a hit or a miss in the configuration left by the victim. We call this process probing the cache.

However, when the attacker probes the cache, the configuration is updated with the new accessed memory blocks which may in the end destroy the original configuration of the cache left by the victim. Therefore, when probing the cache, it has to be done in a way that maximizes the information recovered about the configuration left by the victim before the cache is updated "too far" and the information about the victim is lost.

In this chapter we develop the theory that allows us to characterize how the cache holds information about the victim's execution (the information absorption) and how the attacker tries to recover as much information as possible about the configuration of the cache (the information extraction). Since the set of victim's memory blocks $B_{v}$ is finite, the set of cache configurations is also finite. Then, the results that we obtain are not restricted to cache algorithms but are applicable to any Mealy machine, see Section 2.4.

We also develop an algorithm to compute the information extraction on any cache algorithm. We will use this algorithm to compute upper bounds on information extraction, Chapter 5 , and to improve the security guaranties of CacheAudit, Chapter 6. 


\subsection{Information Absorption}

In this Section we characterize the set of victim's states $\Gamma_{v} \subseteq \Gamma$ (or the set of victim's configurations $G_{v} \subseteq G$ from (3.2) if we restrict to cache algorithms) reachable by a set of traces of input symbols from a given state, give the definition of information absorption and show that computing the amount of absorbed information is not as straightforward as counting the number of elements in the set $\Gamma_{v}$ since some of them are indistinguishable to the attacker.

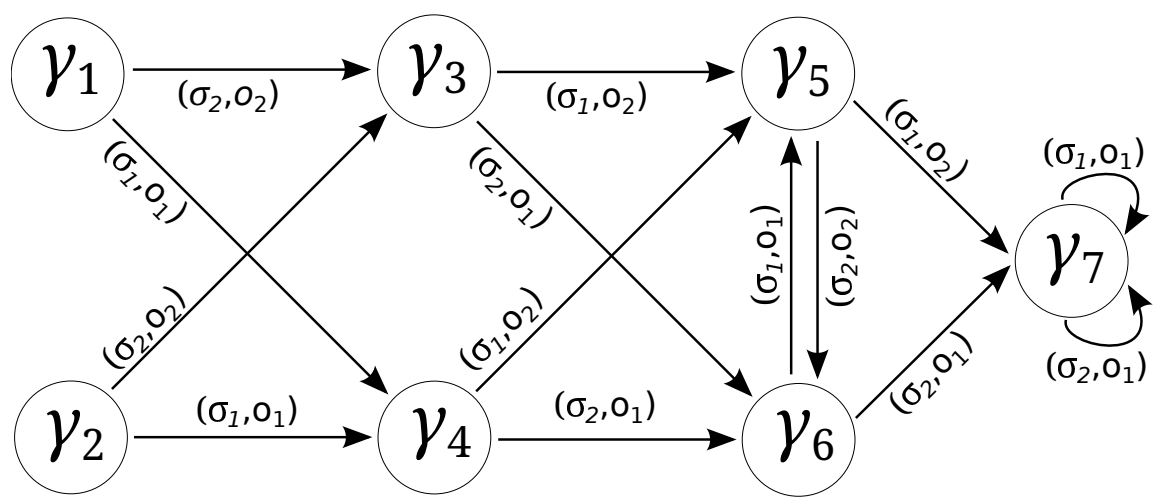

Figure 4.1: Example of a Mealy machine.

Before we give the formal definition we note that the absorbed information depends on two things: the state of the Mealy machine before the victim's computation (not necessarily the initial state of the machine) and the inputs. To see the effect of the state the victim starts of, $\gamma_{v} \in \Gamma$, consider the Mealy machine in Figure 4.1 and assume that the victim may use any sequence of inputs from $\Sigma^{*}=\left\{\sigma_{1}, \sigma_{2}\right\}^{*}$. If the machine starts from the state $\gamma_{v}=\gamma_{7}$ only that one state is reachable, $\Gamma_{v}=\left\{\gamma_{7}\right\}$; if $\gamma_{v} \in\left\{\gamma_{5}, \gamma_{6}\right\}$ then $\Gamma_{v}=\left\{\gamma_{5}, \gamma_{6}, \gamma_{7}\right\}$; if $\gamma_{v} \in\left\{\gamma_{3}, \gamma_{4}\right\}$ then $\Gamma_{v}=\left\{\gamma_{3}, \gamma_{4}, \gamma_{5}, \gamma_{6}, \gamma_{7}\right\}$ and finally if $\gamma_{v} \in\left\{\gamma_{1}, \gamma_{2}\right\}$ then $\Gamma_{v}=\Gamma$.

However, although in the worst case, seven states of the Mealy machine are reachable, note that the pairs of states $\gamma_{1}, \gamma_{2}$ and $\gamma_{3}, \gamma_{4}$ are indistinguishable in the effect they produce. Recall that the only observable behavior of the cache is through the effect function and so, two states are distinguishable if, for some trace of input symbols, the effect function returns a different trace of output symbols for each state. For this reason, the attacker is unable to differentiate $\gamma_{1}$ from $\gamma_{2}$ and $\gamma_{3}$ from $\gamma_{4}$. This corresponds to the notion of equivalent states on a Mealy machine.

Definition 4.1 (Equivalent states). Given a Mealy machine $M$, we say that two states $\gamma, \gamma^{\prime} \in \Gamma$ are equivalent, denoted $\gamma \sim \gamma^{\prime}$, if for every trace of memory blocks $t \in \Sigma^{*}$,

$$
o b s^{*}(\gamma, t)=o b s^{*}\left(\gamma^{\prime}, t\right)
$$


When defining the information absorption based on the set of victim's states, we do not focus on how "big" this set is, as if all configurations are equivalent it will produce one single observation when probed, but rather how "varied" these states are, that is, how many classes of equivalence of $\sim$ are in $\Gamma_{v}$.

Given a set of victim's states $\Gamma_{v} \subseteq \Gamma$, we denote the class of equivalence under $\sim$ of state $\gamma \in \Gamma_{v}$ by

$$
[\gamma]=\left\{\gamma^{\prime} \in \Gamma_{v} \mid \gamma^{\prime} \sim \gamma\right\}
$$

With the definition of classes of equivalence we define the information absorption of a Mealy machine.

Definition 4.2 (Information absorption). We define the absorbed information of a Mealy machine $M=(\Gamma, \Sigma, O, i \gamma, u p, o b s)$ with respect to a state $\gamma_{v}$ and a set of traces of input symbols $T \subseteq \Sigma_{v}^{*}$ as the number of classes of equivalence of $\sim$ in the set of victim's states:

$$
\operatorname{Abs}\left(M, \gamma_{v}, T\right)=\left|\left\{[\gamma] \mid \gamma \in \Gamma_{v}\right\}\right|,
$$

where $\Gamma_{v}=\left\{\gamma \in \Gamma \mid \exists t \in T: u p\left(t, \gamma_{v}\right)=\gamma\right\}$.

For a given program, existing static analysis techniques can be used to compute approximations of the set of traces $T$ and the absorption of a particular cache algorithm, modeled by a Mealy machine $M$. In Chapter 6 we present the results of a static analysis of several cryptographic implementations.

\subsection{Information Extraction}

In this section we characterize the information extraction for different cache replacement policies. For this we develop a novel model that characterizes the information an adaptive attacker can learn about the state of a Mealy machine left by the victim via probing.

Later, we introduce an algorithm to compute the information extraction on any cache algorithm.

\subsubsection{Probing Strategies}

Let $M=(\Gamma, \Sigma, O$, up,obs $)$ be a Mealy machine. A probe $p$ of $M$ is an alternating sequence $p=\sigma_{1} o_{1} \sigma_{2} \ldots \sigma_{n} o_{n}$ of attacker's input symbols $\sigma_{i} \in \Sigma_{a} \subseteq \sum$ and effects $o_{i} \in O$, such that $M$ outputs $o_{1} \ldots o_{n}$ when the trace $\sigma_{1} \ldots \sigma_{n}$ is the input. We say that a state $\gamma \in \Gamma_{M}$ is coherent with probe $p$ if,

$$
o b s^{*}\left(\gamma, \sigma_{1} \ldots \sigma_{n}\right)=o_{1} \ldots o_{n},
$$


i.e., the probe does not exclude $\gamma$ as a potential victim's state of $M$. Note that, if $\gamma$ is coherent with probe $p$, then any $\gamma^{\prime} \sim \gamma$ is also coherent with probe $p$.

Along the lines of [AS07, KB07], we define the attacker's knowledge set $K(p)$ about the initial state of $M$ as the subset of possible states that are coherent with probe $p$.

$$
K(p)=\left\{\gamma \in \Gamma_{v} \mid \gamma \text { is coherent with } p\right\}
$$

For convenience, we also define the attacker's final knowledge set $F K(p)$ as the set of states that $M$ may be in after receiving the inputs and producing the outputs in the probe $p$ :

$$
F K(p)=\left\{u p\left(\gamma, \sigma_{n} \ldots \sigma_{1}\right) \mid \gamma \in K(p)\right\}
$$

An attacker may be able to choose inputs based on previous observations, that is, the probing can be adaptive. To model adaptivity we introduce probing strategies. A probing strategy is a function from a sequence of observations to an input symbol, att : $O^{*} \rightarrow \sigma_{a}$. This way, the first input to make comes from applying the function to the empty sequence, $\sigma_{1}=\operatorname{att}(\varepsilon)$, the second input is a function of the previous observation, $\sigma_{2}=\operatorname{att}\left(o_{1}\right)$, and so, for any $i \sigma_{i}=$ $\operatorname{att}\left(o_{1} \ldots o_{i-1}\right)$. We say that $p$ is a probe of att, if $p$ may be obtained from the probing strategy att.

We now present a toy example that we will use through the section to illustrate the use of probing strategies.

Example 4.3. Consider a Mealy machine where $\Gamma=\Gamma_{v}=\Sigma_{a}=\{0,1, \ldots, 6\}$, the observation and transition functions are:

$$
o b s(\gamma, \sigma)=\left\{\begin{array}{ll}
0 & \text { if } \gamma<\sigma-1, \\
2 & \text { if } \gamma \in[\sigma-1, \sigma+1], \\
1 & \text { if } \gamma+1<\sigma .
\end{array} \quad u p(\gamma, \sigma)= \begin{cases}\gamma+1 & \text { if } \gamma<\sigma, \\
\gamma & \text { if } \gamma \in[\sigma, \sigma+1], \\
\gamma-1 & \text { if } \sigma+1<\gamma .\end{cases}\right.
$$

Consider the probing strategy given by the function $\operatorname{att}\left(o_{1} \ldots o_{n}\right)=0+\sum_{i=1}^{n} o_{i}$, which starts by inputting 0 and determines the next input based on the previous outputs. We will later see that att is a good probing strategy in this example.

By definition, we can apply a probing strategy indefinitely on sequences of arbitrary length and thus probe the Mealy machine indefinitely. However, at some point additional inputs are of no use, as the following definition characterizes.

Definition 4.4. We say that a probe $p=\sigma_{1} o_{1} \sigma_{2} \ldots \sigma_{n} o_{n}$ of probing strategy att is depleted w.r.t. to $a t t$, if for all probes $q$ of att that are extensions of $p$, i.e., $q=p \sigma_{n+1} o_{n+1} \sigma_{n+2} \ldots \sigma_{m} o_{m}$, the knowledge sets are equal, i.e., $K(p)=K(q)$. We say a depleted probe $p=\sigma_{1} o_{1} \sigma_{2} \ldots \sigma_{n} o_{n}$ is of minimal length when, a probe $q$ made of a sub-sequence of it, $q=\sigma_{k_{1}} o_{k_{1}} \sigma_{k_{2}} \ldots \sigma_{k_{i}} o_{k_{i}}$ for any $i<n$, is not depleted. 
We next show that the knowledge sets of depleted probes of a probing strategy form a partition of the states of $M$. That is, the knowledge sets of distinct sequences are pairwise disjoint and their union contains all states.

Proposition 4.5. Given a probing strategy att, the set of all knowledge sets produced by depleted probes w.r.t. att

$$
R_{\text {att }}=\left\{K(p) \mid \text { probe } p=\operatorname{att}(\varepsilon) o_{1} \ldots \text { att }\left(o_{1} \ldots o_{n-1}\right) o_{n} \wedge p \text { is depleted w.r.t. att }\right\},
$$

is a partition of the set of possible states $\Gamma_{v}$.

Proof. We will first prove the following related statement: Let $R_{\text {att }}(i)$ be defined as follows: $R_{\text {att }}(i)=\left\{K(p) \mid\right.$ probe $p=\operatorname{att}(\varepsilon) o_{1} \ldots$ att $\left.\left(o_{1} \ldots o_{i-1}\right) o_{i}\right\}$. Then $R_{a t t}(i)$ is a partition of $\Gamma_{v}$ for all $i$.

We prove this statement by induction on $i$.

Induction basis. For $i=0, R_{\text {att }}(i)=\{K(\epsilon)\}$, and $K(\epsilon)=\Gamma_{v}$. So $R_{\text {att }}(i)$ is a trivial partition of $\Gamma_{v}$.

Induction step. For $i+1$,

$$
\begin{gathered}
R_{a t t}(i+1)=\{ \\
\wedge\left(p \sigma_{i+1} o_{i+1}\right) \mid \text { probe } p=\operatorname{att}(\varepsilon) o_{1} \ldots \operatorname{att}\left(o_{1} \ldots o_{i-1}\right) o_{i} \\
\left.\wedge \sigma_{i+1}=\operatorname{att}\left(o_{1} \ldots o_{i}\right) \wedge o_{i+1} \in \text { obs }\left(F K(p), \sigma_{i+1}\right)\right\}
\end{gathered}
$$

By induction hypothesis $R_{a t t}(i)$ is a partition of $\Gamma_{v}$. It is easy to see that for each probe $p$ of length $i, K(p)$ is partitioned by $\left\{K\left(p \sigma_{i+1} o_{i+1}\right) \mid \sigma_{i+1}=\operatorname{att}\left(o_{1} \ldots o_{i}\right) \wedge\right.$ $\left.o_{i+1} \in \operatorname{obs}\left(F K(p), \sigma_{i+1}\right)\right\}$. So $R_{a t t}(i+1)$ is a refinement of $R_{a t t}(i)$ and thus also a partition of $\Gamma_{v}$.

Let $n$ be the length of the longest depleted probe of minimal length. Then $R_{\text {att }}=R_{\text {att }}(n)$ as all probes considered in $R_{\text {att }}(n)$ must be depleted, and as extensions of depleted probes have the same knowledge set as their corresponding depleted probes of minimal length.

Before starting the probing, the attacker knows that the victim's state is an element of the set $\Gamma_{v}$. As the attacker makes inputs and refines the knowledge sets, it reduces the number of coherent states and thus learns information about the victim's initial state. As depleted probes correspond to unrefinable knowledge sets, there is no point in further queries once a probe is depleted.

When constructing a strategy, the attacker needs to consider all the possible outputs that he might observe when eventually applying his strategy. Once all the knowledge sets obtained from an attack strategy cannot be further refined by additional queries, the probes are depleted and the attacker has along the way obtained the finest partition of the set $\Gamma_{v}$ under that strategy and all possible extensions. 


\begin{tabular}{|lll|lllllll|}
\hline $0 / 0$ & & $1 / 1$ & $\mathbf{0}$ & $2 / 2$ & $3 / 3$ & $4 / 4$ & $5 / 5$ & $6 / 6$ \\
\hline $0 / 0$ & 2 & $1 / 1$ & $2 / 1$ & $3 / 2$ & $\mathbf{1}$ & $4 / 3$ & $5 / 4$ & $6 / 5$ \\
\hline $0 / 1$ & $1 / 2$ & $2 / 1$ & $\mathbf{3}$ & $3 / 2$ & $4 / 2$ & $5 / 3$ & 2 & $6 / 4$ \\
\hline $0 / 1$ & $1 / 2$ & $2 / 2$ & $3 / 3$ & $4 / 2$ & 4 & $5 / 3$ & $6 / 3$ \\
\hline $0 / 1$ & $1 / 2$ & $2 / 2$ & $3 / 3$ & $4 / 3$ & $5 / 4$ & $6 / 3$ \\
\hline
\end{tabular}

Table 4.1: Partition from Example 4.6.

Example 4.6. Following Example 4.3 we apply the probing strategy to the set of possible states and obtain the partition shown in Table 4.1. Each row shows the knowledge sets before and after the elements are updated (left and right, respectively). The first row shows the initial knowledge set, i.e., $\Gamma_{v}$. The bold face 0 indicates the first input symbol, which partitions the initial knowledge set into two knowledge sets, corresponding to the two possible outputs of the Mealy machine on the input 0 . For each resulting knowledge set, except for the singleton ones where the probes are depleted, the figure then indicates the next input following the probing strategy and how it partitions its knowledge set. After at most four inputs we obtain a partition of all singleton knowledge sets.

For every attack strategy there is a finite set of depleted probes of minimal length. We define $Y_{a t t}$ from Theorem 3.1 as the random variable that captures the sequence of observations obtained when following probing strategy att until obtaining a depleted probe of minimal length. So $\operatorname{ran}\left(Y_{a t t}\right) \subseteq O^{*}$ is the set of sequences of observations obtained from the depleted probes of minimal length of att. Every depleted probe corresponds to a knowledge set; so we can relate every element of $\operatorname{ran}\left(Y_{a t t}\right)$ to a knowledge set. Therefore, computing $\left|\operatorname{ran}\left(Y_{a t t}\right)\right|$ is equivalent to counting the number of knowledge sets in the partition induced by the strategy att.

Definition 4.7. We say that a strategy att is optimal if the partition $R_{\text {att }}$ it induces on a set of possible states $\Gamma_{v}$, has the maximal number of knowledge sets among all strategies. We call this number $\mu$ the maximum information leakage.

The strategy presented in Example 4.3 is actually optimal since no partition can be better than the one that produces singleton knowledge sets. On the other hand, the strategy $\operatorname{att}\left(o_{1} \ldots o_{n}\right)=1+\sum_{i=1}^{n} o_{i}$ is not optimal since the first input, 1 , is not able to distinguish the initial states 0 and 1 , which are both updated to 1 as a result of the input, $u p_{1}(0)=u p_{1}(1)=1$, and so they can not be distinguished by this strategy.

\subsubsection{An Algorithm for Information Extraction}

In this section we present an algorithm for computing the maximum information leakage $\mu$ for a given cache algorithm $\mathrm{P}$. The algorithm complements 
Propositions 5.17 to 5.20 in that it can deliver $\mu$ for a specific set of configurations $\Gamma_{v} \subseteq \Gamma$ and an arbitrary cache algorithm. We use it later to compute extraction with respect to a given memory footprint, and to replace the engine for counting configurations in the CacheAudit static analyzer, leading to tighter bounds on the leakage.

In principle, our algorithm works for all types of mealy machines. It enumerates all attack strategies att and computes their partitions $R_{\text {att }}$ by grouping states in $\Gamma_{v}$ according to the corresponding observations. Additionally, we use two techniques for improving efficiency and ensuring termination:

- First, instead of maintaining the knowledge sets $K(p)$, for every probe $p$, we maintain the final knowledge set $F K(p)$. Using the final knowledge set enables us to track the number of original knowledge sets, as required for computing leakage. At the same time it enables re-use of the computation leading to $F K(p)$ across different strategies.

- Second, we need to identify cycles when refining partitions in order to ensure termination. We say that a probe $q$ is redundant w.r.t another probe $p$, if $F K(p q)=F K(p)$. That is, the probe $q$ does not further refine the (final) knowledge set of $p$. The probe $q$ represents a cycle, which we detect by keeping track of already visited final knowledge sets.

The pseudocode is given in Algorithm 1. We next argue its correctness.

Proposition 4.8. Given a Mealy machine $M=(\Gamma, \Sigma, O, i \gamma$, up,obs $)$, Algorithm 1 terminates and finds the maximum information leakage $\mu$ for a set of possible states $\Gamma_{v}$.

Proof. The algorithm recursively studies all the possible sequences of inputs. In every call, it cycles through all the inputs (line 7 ) and for each output of the observation function (line 16), partitions the set into final knowledge sets (line 17), updates every final knowledge set (line 18) and recursively calls again the function (line 19). Then, each execution obtains from the following calls the best way to partition the final knowledge set for each input (line 19), chooses the one with the maximum value (line 21), and returns it to the previous level (line 23). Our algorithm terminates if the number of knowledge sets is equal to the size of the set of possible states (line 8), at which point the knowledge sets cannot be further refined.

The flag sets $\mathcal{F}$ are used to keep track of the redundant sequences. Whenever an input does not partition the set (i.e. there is only one observation), it is saved (line 11). If a later call sees that the updated set $\Gamma$ is equal to one saved in $\mathcal{F}$ then that call has produced a redundant sequence and so it is stopped with one final knowledge set (line 3 ). This procedure also guarantees that the algorithm terminates. If all the inputs that only produce one observation result in a redundant sequence, the algorithm is forced to choose one that partitions 


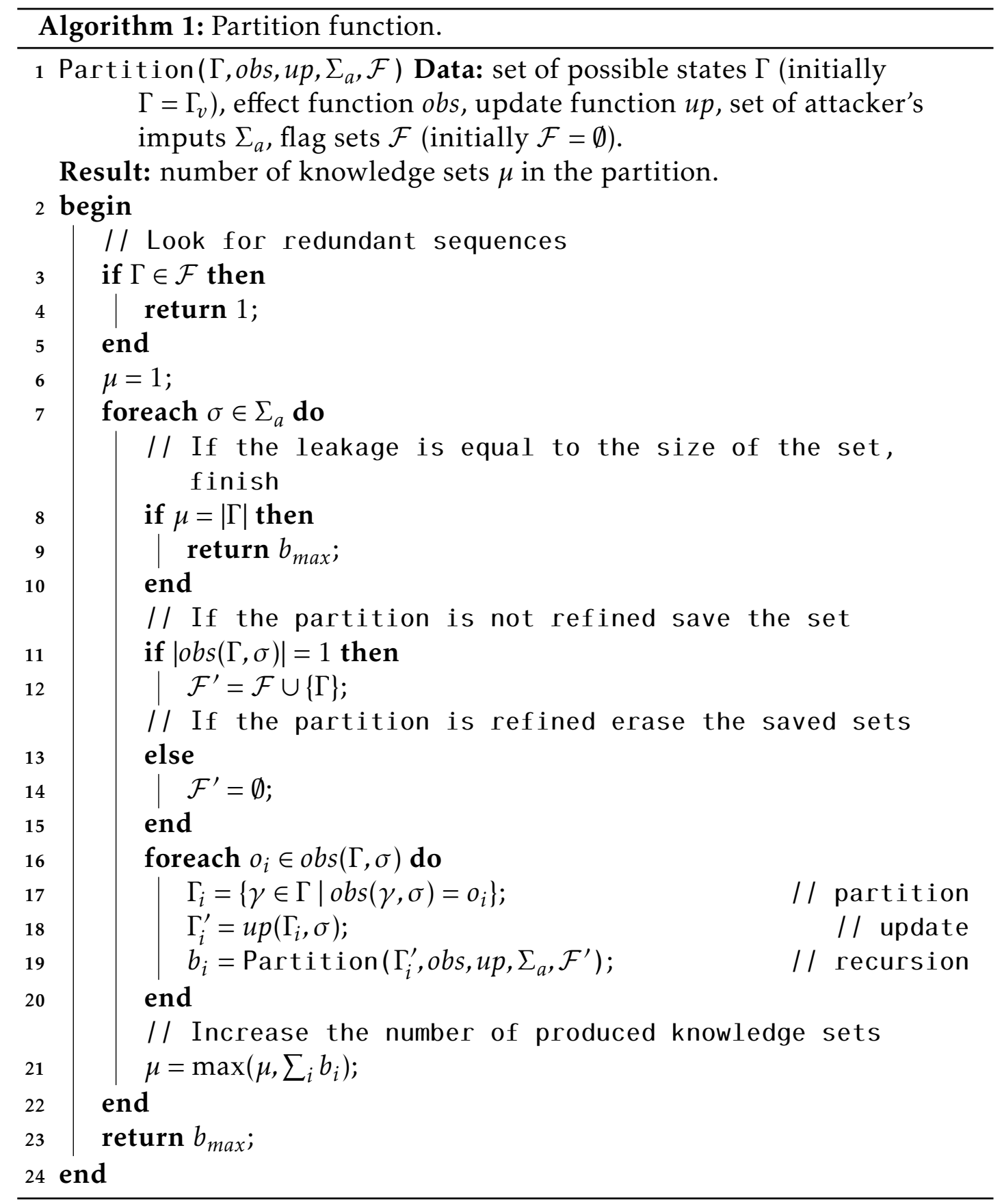


the set and eventually depletes the probes. Once a probe is depleted, the algorithm does not extend it anymore since every extension of a depleted probe is redundant. 


\section{CHAPTER 5}

\section{UPPER BOUNDS ON LEAKAGE}

When analyzing the performance of an algorithm, one desirable characteristic of the conclusions we obtain is that they are independent of any input we give to the algorithm. Therefore, we look for results on the performance of the algorithm that are satisfied by every possible input of the program.

One basic approach to obtain a performance result independent of the algorithm is to estimate the largest value of the performance of the algorithm, every input will produce a performance value bellow the estimated largest value. This procedure is known as worst-case analysis. It is widely used, for example, for hard real-time systems which need to satisfy stringent timing constraints that depend on the systems they control [AMWH94, WEE ${ }^{+}$08].

In the case of security of side channels, a bigger set of observations means less security guaranties for the algorithm. Then, the worst-case scenario is the program (i.e. the set of traces of memory blocks) that produces the largest number of observations.

One important property of all the cache algorithms studied here is that the actual memory blocks used by the victim are irrelevant to obtain the leakage of the worst-case scenario, the only important property about the set of memory blocks is its size. We call this number the footprint and denote it by $f p=|B|$. This terminology is loosely connected with the notion of a memory footprint as used in the theory of locality [XDLB13]. Theory of locality defines the footprint as the number of distinct memory blocks accessed during a time window, i.e. on a trace of a given length. In our cases we consider a finite set of memory blocks so the trace is the whole execution of the program.

This property holds for a different class of cache algorithms (automata-based or permutation-based) depending on the attacker. Throughout the chapter we will see how it is obtained for each attacker.

For the time and trace-based attackers, we obtain the same worst-case leakage for any algorithm represented with the automata model. 
For the access-based attacker, we analyze three permutation-based cache algorithms, LRU, FIFO and PLRU on fully associative caches. We obtain values for the worst-case absorption depending on the configuration of the cache and the footprint. We show that the information extraction for LRU and FIFO is always bounded by a constant that depends on the associativity of the cache and that for PLRU the upper bound grows as we increase the footprint. We verify the upper bounds on information extraction by applying Algoritm 1.

Moreover, we analyze set-associative caches for the access-based attacker and show that not always distributing the memory blocks over several cache sets guaranties a reduction in leakage.

\subsection{Time and Trace-based Attackers}

Time and trace-based attackers monitor the effect of the trace of memory blocks in the cache. A trace attacker observes whether each individual memory block from the trace produces a hit or a miss whereas a time attacker observes the total number of misses the trace of memory blocks produces, without knowing which particular memory blocks produced the misses.

Note that, by looking at the update function in the automata-based model (2.1), one can see that the only dependance on the memory blocks lies in whether they are cached or not:

- If a memory block is cached, regardless of which block it is or the line where it is cached, accessing it produces a hit for any cache algorithm.

- If a memory block is not cached, regardless of which memory block it is, accessing it produces a miss for any cache algorithm.

Then, as long as there are memory blocks cached and not cached for every configuration, a new access to a memory block can be either a hit or a miss.

Since the sets of observations obtained for the time and trace-based attackers grow with the length of the traces of memory blocks, we abstract from programs to sets of traces of equal length and use the length of the traces to establish a worst-case scenario analysis.

Given a set of memory blocks $B$ and a length of the trace $l$, we obtain the largest set of observations by considering the set of all possible traces of length $l$ using the memory blocks from $B$, that is, the set of traces $B^{l}$. This way, for every cache algorithm $P$ the number of observations that $P$ produces on a set of traces of length $l, T \subseteq B^{l}$, is upper bounded by the observations produced on the whole set $B^{l}$.

However, we saw above that the effect a memory block has on the cache does not depend on the block itself but only on whether the block is cached or not. This allows us to abstract from the set $B^{l}$ and express the leakage of the 
worst-case scenario for the time and trace-based attacker using only the length of the traces and the size of $B$, that is, the footprint.

Then, given a footprint $f p$, a length $l$ and a configuration $g$ the leakage of the worst-case scenario for the time and trace attacker is, respectively:

$$
\begin{aligned}
& \mathrm{P}_{\mathrm{tme}}^{\max }(f p, l, g)=\left|\mathrm{P}_{\mathrm{tme}}\left(B^{l}, g\right)\right|, \\
& \mathrm{P}_{\mathrm{trc}}^{\max }(f p, l, g)=\left|\mathrm{P}_{\mathrm{trc}}\left(B^{l}, g\right)\right|,
\end{aligned}
$$

where $|B|=f p$.

For any cache algorithm $P$ we denote the fill function, fill : $G \rightarrow \mathbb{N} \cup\{0\}$, as the function that, given a configuration, returns the number of lines that hold a valid memory block:

$$
\text { fill }((s, c))=|\{j \leq \eta \mid c(j) \neq \perp\}| .
$$

Using the fill function we get the number of observations obtained from a configuration when using the set of traces $B^{l}$.

Theorem 5.1. For any cache algorithm $\mathrm{P}$ and any configuration $g \in G_{\mathrm{P}}$ different from the initial one, the number of observations a time and trace-based attacker produces on the set of traces $B^{l}$ is:

$$
\begin{aligned}
& \mathrm{P}_{\mathrm{tme}}^{\max }(f p, l, g)= \begin{cases}\min (f p-f i l l(g), l) & \text { if } f p \leq \eta, \\
l+1 & \text { if } f p>\eta .\end{cases} \\
& \mathrm{P}_{\mathrm{trc}}^{\max }(f p, l, g)= \begin{cases}\sum_{m=0}^{\min (f p-f i l l(g), l)} \frac{l !}{m !(l-m) !} & \text { if } f p \leq \eta, \\
2^{l} & \text { if } f p>\eta .\end{cases}
\end{aligned}
$$

For the initial configuration ig $\in G_{\mathrm{P}}$, the number of observations a time and trace-based attacker produces on the set of traces $B^{l}$ is:

$$
\begin{aligned}
& \mathrm{P}_{\mathrm{tme}}^{\max }(f p, l, g)= \begin{cases}\min (f p-1, l-1) & \text { if } f p \leq \eta, \\
l & \text { if } f p>\eta .\end{cases} \\
& \mathrm{P}_{\mathrm{trc}}^{\max }(f p, l, g)= \begin{cases}\sum_{m=0}^{\min (f p-1, l-1)} \frac{(l-1) !}{m !(l-1-m) !} & \text { if } f p \leq \eta, \\
2^{l-1} & \text { if } f p>\eta .\end{cases}
\end{aligned}
$$

Proof. For almost all configurations and sets of memory blocks, a new memory block can produce either a hit or a miss:

- For any configuration different from the initial one $(c, s) \neq i g$, there exists line and a memory block $b$ such that $c(j)=b$, that is, the memory block $b$ is cached. Accessing $b$ again produces a hit. 
- Given a configuration $g=(s, c)$, if $f p>f i l l(g)$, there exists a memory block such that, for all $j \leq \eta, c(j) \neq b$, that is, the memory block $b$ is not cached. Accessing $b$ produces a miss.

Note that both cases are independent of the actual memory blocks except for the size of the set in the second case. This allows to use the footprint, $f p=|B|$, as a generalization of the size of the set.

Also, for every configuration $g$ and memory block $b$, fill $(u p d(g, b))=$ fill $(g)$ if $b$ produces a hit and fill $(u p d(g, b))=f i l l(g)+1$ if $b$ produces a miss

First we consider a configuration $g$ different from the initial configuration. From this configuration, if $f p \leq \eta$, any trace of memory blocks of length $l$ produces between zero and $\min (f p-f i l l(g), l)$ misses and these misses can occur at any point in the trace.

A time-based attacker observes the number of misses produced by the trace which leads to

$$
P_{\text {tme }}^{\max }(f p, l, g)=\min \left(f p-f_{i l l}(g), l\right) \text {. }
$$

A trace-based attacker observes the distribution of misses over the trace. For a fixed number of misses $m$, the number of distinct traces of length $l$ with exactly $m$ misses is $\left(\begin{array}{l}l \\ m\end{array}\right)$. Then

$$
\mathrm{P}_{\mathrm{trc}}^{\max }(f p, l, g)=\sum_{m=0}^{\min (f p-f i l l(g), l)} \frac{l !}{m !(l-m) !},
$$

If $f p>\eta$, any trace of memory blocks of length $l$ produces between zero and $l$ misses and these misses can occur at any point in the trace. This leads to $\left|P_{\text {tme }}\left(B^{l}, g\right)\right|=l+1$ for the time-based attacker and $\left|P_{\text {trc }}\left(B^{l}, g\right)\right|=2^{l}$ for a tracebased attacker.

Now we consider the case of the initial configuration, ig. In this case, the first access is always a miss but the updated configuration behaves like in the previous case. Therefore the number of observations obtained from the initial configuration and a trace of memory blocks of length $l$ is the same as the number of observations obtained from a configuration $g$ with fill $(g)=1$ and a trace of memory blocks of length $l-1$.

For any trace of memory blocks, each memory block produces at most two different effects on the cache, a hit or a miss. Then the number of misses any cache algorithm can produce on a trace of memory blocks is positive and upper bounded by the length of the trace. Therefore, for any cache algorithm $\mathrm{P}$, set of traces $T \in B^{l}$ and configuration $g \in G$ we have that:

$$
\begin{aligned}
\left|P_{\text {tme }}(g, T)\right| & \leq l+1 . \\
\left|P_{\text {trc }}(g, T)\right| & \leq 2^{l} .
\end{aligned}
$$


Note that these bounds coincide with the second case of (5.3) and (5.4) respectively.

Moreover, apart from the fill function and the capacity, the worst-case number of observations obtained does not depend on the cache algorithm. Therefore we have the following auxiliary result.

Corollary 5.2. Given two cache algorithms $\mathrm{P}, \mathrm{Q}$ such that $\eta_{\mathrm{P}}=\eta_{\mathrm{Q}}$ and two configurations $g_{\mathrm{P}} \in G_{\mathrm{P}}$ and $g_{0} \in G_{0}$ such that fill ${ }_{\mathrm{P}}\left(g_{\mathrm{P}}\right)=$ fill $_{0}\left(g_{0}\right)$, we have:

$$
\begin{aligned}
& P_{\text {tme }}^{\max }(f p, l, g)=Q_{\text {tme }}^{\max }(f p, l, g), \\
& P_{\text {trc }}^{\max }(f p, l, g)=Q_{\text {trc }}^{\max }(f p, l, g) .
\end{aligned}
$$

Then, if two cache algorithms have the same capacity and start updating configurations equally filled, they will produce the same worst-case leakage.

\subsection{Access-based Attacker}

The access-based attacker obtains information not about the effect of the victim's program on the cache but about the final configuration of the cache after the victim's program has finished. This process is not as simple as directly observing the cache but actually requires that the attacker probes the cache, that is, accesses new memory blocks, observes the effect they have on the cache and draws conclusions about the configuration left by the victim.

In this Section we focus on permutation-based cache algorithms and so we will use the permutation-based model introduced in Section 2.2. We will see that, for permutation-based cache algorithms, the information absorption can be computed without considering classes of equivalence as in Section 4.1 and does not depend on set of memory blocks except for its size, i.e. the footprint. We will obtain upper bounds on the information absorption and information extraction for LRU, FIFO and PLRU. We will also apply Algorithm 1 to these three cache algorithms.

As we saw on Section 2.2, permutation-based cache algorithms behave independently on each cache set. For this reason, we first show how the absorption and extraction can be computed on fully associative caches, Sections 5.2.1 and 5.2.2, later on we will extend these results to set-associative caches and we will see how, depending on the memory demand, several cache sets can be counterproductive for the security.

\subsubsection{Information Absorption}

Given a cache algorithm $\mathrm{P}$, a set of traces $T$ and a configuration $\hat{g}_{v}$, the information absorption is defined based on the set of reachable configurations obtained by updating $\hat{g}_{v}$ with the traces from $T$, see Definition 4.2. 
Given a finite set of memory blocks $B$, the set of possible configurations of algorithm $\mathrm{P}$ is also finite. Then the set of configurations reachable from $\hat{g}_{v}$ by a set of traces $T \subset B^{*}$ is contained in the set of configurations reachable by any trace on the set $B^{*}$. Therefore, to compute the worst-case scenario information absorption, we abstract from programs using blocks from $B$ to the set of all possible traces using those blocks, $B^{*}$.

Moreover, computing the absorption when dealing with permutation-based cache algorithms is simpler due to two properties:

1. All classes of equivalence are singleton so computing the information absorption reduces to counting the number of reachable configurations.

2. Given a configuration $\hat{g}_{v}$ and a set of victim's memory blocks $B_{v}$, the information absorption only depends on the blocks from $B_{v}$ cached in $\hat{g}_{v}$ and the size of $B_{v}$.

In this Section we explain how these two properties work and obtain results for the information absorption for LRU, FIFO and PLRU depending on the configuration $\hat{g}_{v}$.

\section{No Equivalent Configurations}

As we saw in the previous chapter, the information absorption is computed by counting the classes of equivalence of the set of reachable configurations, Definition 4.2. However, the following lemma shows that, when considering permutation-based cache algorithms, all classes of equivalence are singleton.

Lemma 5.3. Given a permutation-based cache algorithm $\mathrm{P}$ and a set of memory blocks $B$ larger than the associativity, $|B|>A$, the set of configurations $\widehat{G}$ does not have a pair of equivalent configurations.

Proof. Let $\hat{g} \in \widehat{G}$ be a configuration. We show that, for any other configuration $\hat{g}^{\prime} \neq \hat{g}$, we have that $\hat{g}^{\prime} \neq \hat{g}$.

Consider that $\hat{g}$ and $\hat{g}^{\prime}$ do not have the same memory blocks cached, that is, there exist $b$ such that $\hat{g}(b)<A$ but $\hat{g}^{\prime}(b)=A$. Then, we have that $e f f(\hat{g}, b) \neq$ eff $\left(\hat{g}^{\prime}, b\right)$ and so $\hat{g}^{\prime} \nsucc \hat{g}$.

Consider that both configurations have the same blocks cached, that is, for every memory block $b, \hat{g}(b)<A \Leftrightarrow \hat{g}^{\prime}(b)<A$. However, since the configurations are different, the memory blocks are not mapped to the same ages in both configurations. Consider the largest age $a$ that has a different block for each configuration, that is, there exist two memory block $b \neq b^{\prime}$ such that $\hat{g}(b)=$ $\hat{g}^{\prime}\left(b^{\prime}\right)=a$ and, for any $\tilde{a}>a$ there exists a memory block $\tilde{b}$ such that $\hat{g}(\tilde{b})=$ $\hat{g}^{\prime}(\tilde{b})=\tilde{a}$.

Now we access a trace of memory blocks $b_{1} \ldots b_{A-a}$ to produce misses in both configurations at the same time. These blocks always exist: 
- Memory block $b_{1}$ is any of the blocks from

$$
B \backslash\left\{b_{0}^{\prime}, \ldots, b_{A-1}^{\prime}\right\},
$$

where $\hat{g}\left(b_{i}^{\prime}\right)=i$. Since $|B|>A$, this set is non-empty.

- Memory block $b_{i}$ for $i \geq 2$ is any of the blocks from

$$
B \backslash\left\{b_{0}^{\prime}, \ldots, b_{A-1}^{\prime}\right\} \backslash\left\{b_{1}, \ldots, b_{i-1}\right\} \cup\left\{b_{A-i+1}^{\prime}, \ldots, b_{A-1}^{\prime}\right\} .
$$

Even if $|B|=A+1$, each memory block that produces a miss evicts the same memory block from both configurations, thus providing a new block to produce the next miss.

This trace increases, with every memory block, the ages of the blocks $b$ and $b^{\prime}$ until evicting them.

Then $\widehat{u p d}\left(\hat{g}, b_{1} \ldots b_{A-a}\right)$ and $\widehat{u p d}\left(\hat{g}^{\prime}, b_{1} \ldots b_{A-a}\right)$ do not have memory blocks $b$ and $b^{\prime}$ respectively. Accessing any of them will produce a different effect on the updated configurations. Then, we have that

$$
e f f\left(\hat{g}, b_{1} \ldots b_{A-a} b\right) \neq e f f\left(\hat{g}^{\prime}, b_{1} \ldots b_{A-a} b\right),
$$

and so $\hat{g}^{\prime} \nmid \hat{g}$.

Since we always consider that the set of attacker's memory blocks is as big as the capacity of the cache algorithm, this result will always hold and therefore we compute the information absorption simply by counting the number of reachable configurations.

\section{Data Independence}

We now see that the actual memory blocks the cache uses are irrelevant for computing the information absorption. This allows to compute the information absorption taking into account only how filled the configuration is and how many memory blocks the cache uses, that is, its footprint.

We begin by defining the two extreme cases of configuration types that are relevant to the information absorption.

Definition 5.4. Given a permutation-based cache algorithm, P, we say that a configuration $\hat{g}: B \rightarrow\{0, \ldots, A\}$ is

1. empty w.r.t. $\tilde{B} \subseteq B$ if $\hat{g}(\tilde{B})=\{\hat{g}(b) \mid b \in \tilde{B}\}=\{A\}$. That is, none of the blocks in $\tilde{B}$ are cached.

2. filled with $\tilde{B}$ if $c(\tilde{B})=\{0, \ldots, \min (A,|\tilde{B}|-1)\}$. That is, the blocks in $\tilde{B}$ occupy the cache. If $\tilde{B} \subseteq B$ contains less blocks than cache lines, we require that the first $|\tilde{B}|$ lines are filled. 
The notions of empty and filled configurations are relative to a set of memory blocks. We will consider empty and filled configurations relative to the memory blocks accessed by the victim, $B_{v}$.

Consider a permutation-based cache algorithm $\mathrm{P}$ and a partially filled configuration $\hat{g} \in \widehat{G}$, that is, where there is a trace of distinct memory blocks $b_{0} \ldots b_{n}$ with $n \leq \min \left(A,\left|B_{v}\right|-1\right)$ such that $\hat{g}\left(b_{i}\right)=i$ for $i \leq n$. Then, any configuration reachable from $\hat{g}$ by accessing a trace $t \in B_{v}^{*}$ is reachable from an empty one $\hat{g}_{e}$ with the trace $t^{\prime}=b_{n} \ldots b_{0} t$. Since $\hat{g}_{e}$ is empty, we load the blocks $b_{0} \ldots b_{n}$ in reverse order; these access produce misses and so, after the updates, $\widehat{u p d}\left(\hat{g}_{e}, b_{0} \cdots b_{n}\right)\left(b_{i}\right)=i$ for every $i \leq n$, see (2.4). Therefore $A b s\left(\mathrm{P}, \hat{g}, B_{v}^{*}\right) \leq$ $A b s\left(\mathrm{P}, \hat{g}_{e}, B_{v}^{*}\right)$. Using this argument we can see that, for the same set of memory blocks, the value of the absorbed information is the smallest when starting on a filled configuration and is the largest when starting on an empty configuration.

The following result is central for our program-independent analysis of permutation-based cache algorithms. It shows that absorption can be characterized independently of the particular set of blocks $B$ that the victim may access:

Theorem 5.5. For any permutation-based cache algorithm $\mathrm{P}$, whenever $\left|B_{1}\right|=\left|B_{2}\right|$, and $\hat{g}_{1}$ is empty (filled) w.r.t. $B_{1}$ and $\hat{g}_{2}$ empty (filled) w.r.t. $B_{2}$, then

$$
A b s\left(\mathrm{P}, \hat{g}_{1}, B_{1}^{*}\right)=A b s\left(\mathrm{P}, \hat{g}_{2}, B_{2}^{*}\right) \text {. }
$$

The proof of Theorem 5.5 follows from the following lemma and the observation that one can define bijections between all sets of equal cardinality.

Lemma 5.6. Given permutation-based cache algorithm $\mathrm{P}$, let $f: B \rightarrow B$ be a bijection. Then, for any configuration $\hat{g}$ :

$$
A b s\left(\mathrm{P}, \hat{g}, \tilde{B}^{*}\right)=A b s\left(\mathrm{P}, \hat{g} \circ f^{-1},(f(\tilde{B}))^{*}\right) \text {. }
$$

With $\tilde{B} \subseteq B$.

Proof. For any configuration $\hat{g}$ and any memory blocks $b, b^{\prime}$ we have

$$
\left.\widehat{u p d}(\hat{g}, b) b^{\prime}\right)=\widehat{u p d}\left(\hat{g} \circ f^{-1}, f(b)\right)\left(f\left(b^{\prime}\right)\right) \text {. }
$$

This is because the transition functions of caches do not consider the block itself, they only perform equality checks or compare the ages. Since $f$ is a bijection and $\hat{g} \circ f^{-1}(f(b))=\hat{g}(b)$ for all $b$, the output of the update functions coincides. Therefore, every update from $\hat{g}$ with any trace of blocks $b_{1} \ldots b_{n}$ produces the same configuration as updating $\hat{g} \circ f^{-1}$ with the trace $f\left(b_{1}\right) \ldots f\left(b_{n}\right)$. 
An important consequence of Theorem 5.5 is that, given an identical status, i.e. empty or filled, of the initial configuration, the amount of absorbed information depends only on the number of blocks in $B_{v}$, that is, the footprint. This motivates the specialization of the definition of the absorbed information in terms of the footprint, namely:

$$
A b s_{x}(\mathrm{P}, f p)=A b s\left(\mathrm{P}, \hat{g},\left(B_{v}\right)^{*}\right),
$$

where $\left|B_{v}\right|=f p$. where we use the subscript $x=e m p$ to denote that $\hat{g}$ is empty w.r.t. $B_{v}$, and $x=f l l$ to denote that $\hat{g}$ is filled w.r.t. $B_{v}$.

This results are not necessarily exclusive to permutation-based cache algorithms. Whenever the update function does not depend on the memory block itself but rather on the line that holds the memory block, similar results can be obtained. However, since in the automata-based model the evict function (2.1) does depend on the accessed block, we can not generalize the data independence property to all the cache algorithms represented by the automata-based model.

\section{Results for Filled Caches}

For some permutation-based cache algorithms, when configuration $\hat{g}$ is filled and the footprint is small enough, some configurations are unreachable from $\hat{g}$, which reduces the information absorption. The details for each algorithm are given below. In case every configuration of the cache is reachable, we count all the possible feasible mappings of $f p$ blocks to the set of ages $\{0, \ldots, A\}$. Then the absorbed information is the number of $k$-permutations of $n$ of the memory blocks, i.e., the number of different ordered arrangements of $f p$ memory blocks in a sequence of up to $A$ elements.

Proposition 5.7 (Information absorption for LRU on a filled configuration). For LRU, the absorbed information for a filled cache is:

$$
A b s_{f l l}(\mathrm{LRU}, f p)= \begin{cases}f p ! & \text { if } f p<A, \\ \frac{f p !}{(f p-A) !} & \text { if } f p \geq A .\end{cases}
$$

Proof. Every configuration is reachable, even if starting from a filled one. Since upon a hit the accessed memory block obtains age zero, any configuration can be reached simply accessing the target configuration's blocks from oldest to youngest, see (2.6). Therefore, the absorbed information is the number of $\mathrm{k}$ permutations of $\mathrm{n}$ of the available blocks. If $f p<A$, all the victim's blocks fit in the cache and the k-permutations of $\mathrm{n}$ are $f p$ !. If $f p \geq A$, we can only fit $A$ memory blocks in the cache, which gives the value $f p ! /(f p-A) !$. 
Proposition 5.8 (Information absorption for FIFO on a filled configuration). For F IF0, the absorbed information for a filled configuration is:

$$
A b s_{f l l}(\mathrm{~F} \mathrm{IFO} 0, f p)= \begin{cases}1 & \text { if } f p \leq A, \\ A+1 & \text { if } f p=A+1, \\ \frac{f p !}{(f p-A) !} & \text { if } f p>A+1 .\end{cases}
$$

Proof. An important property of FIFO is that it does not reorder cached blocks upon hits, see (2.5). So, for $f p \leq A$ and a filled configuration, all the accesses are hits and leave the configuration in its original form.

When $f p=A+1$ every reachable configuration contains all but one of the $f p$ many blocks. For a set of blocks $\left\{b_{1}, \ldots, b_{A+1}\right\}$ assume that the cache is initially in the following configuration:

$$
\left[b_{1}, b_{2}, \ldots, b_{A}\right],
$$

where the leftmost element of the list has age zero and the one on the right is the oldest. An access to blocks $b_{1}, \ldots, b_{A}$ results in a hit and leaves the configuration as it is. The only way to change the configuration is by accessing $b_{A+1}$ and causing a miss, which results in the following configuration:

$$
\left[b_{A+1}, b_{1}, b_{2}, \ldots, b_{A-1}\right] .
$$

Again the only way to change the configuration is by accessing $b_{A}$. After that it can only be changed by accessing $b_{A-1}$, then $b_{A-2}$, and so on until reaching again the initial configuration. By doing this we are just cycling over the blocks, always evicting them in the same order. This produces $A+1$ distinct reachable configurations.

If $f p \geq A+1$ we prove we can reach every target configuration by accessing a specific trace of memory blocks. Now there are two blocks outside the cache so we use one for cycling as before, having always an extra block outside. For illustration purposes we take the initial configuration $\left[b_{1}, b_{2}, \ldots, b_{A}\right]$, and the target configuration $\left[b_{A}, \ldots, b_{2}, b_{1}\right]$. We use an algorithmic approach to reach the target configuration from the initial one.

We proceed from oldest to youngest block of the target configuration. First we cycle the cache until the second youngest target block, $b_{2}$, is evicted, we call this the stored block. After that we continue to cycle the cache but without using the stored block, so it is not updated into the cache until we want to. When the oldest target block, $b_{1}$, is placed at the beginning of the cache, we access the stored block. This way we obtain the two oldest blocks of the target configuration in the target order $b_{2}, b_{1}$.

We cycle again until the third youngest target block, $b_{3}$, is evicted and cycle again until the second youngest $b_{2}$, is at the beginning. Then we access $b_{3}$ and obtain the three oldest blocks in the target order. We proceed in the same manner until the target configuration is reached. 
Proposition 5.9. For PLRU, the absorbed information for a filled cache is:

$$
A b s_{f l l}(\text { PLRU, } f p)= \begin{cases}2^{f p-1} & \text { if } 1 \leq f p \leq A, \\ \frac{f p !}{(f p-A) !} & \text { if } f p>A .\end{cases}
$$

Proof. Recall that, conceptually, PLRU maintains a binary tree with the blocks at the leaves and arrows on each non-leaf node pointing to one of the children, see Section 2.2. Since the initial configuration is filled, all the victim's blocks are stored in some leaves of the tree.

If $f p \leq A$, all the accesses are going to produce hits, and the only update is a permutation of the ages which, in the tree representation, is the flipping of the arrows away from the accessed block. Then, the number of reachable configurations depends on how many of these arrows can point in more than one direction. An arrow may point in any direction if its children both have at least one victim's block that can be used to flip the arrow. So we we need to determine the number of non-leaf nodes where the two children have each at least one block.

Following the tree from root to leaves we can view the internal nodes with victim's blocks at the leaves reachable from both of its children as partitions of the set of victim's blocks into two subsets. This way, counting nodes with blocks in both children is equivalent to counting the number of partitions that can be performed until we obtain all singleton subsets. The amount of times we can partition a set of $f p$ elements into two subsets until obtaining singleton subsets is $f p-1$, independently of how the partitions are done. Therefore, there are $f p-1$ arrows that may point to any direction. This produces $2^{f p-1}$ reachable configurations.

If $f p>A$ we prove that every configuration is reachable from an initial one by accessing a trace of blocks. For this we consider the target configuration as a tree with the blocks placed in specific leaves and the arrows in specific orientations. We divide the proof in two parts, first the case where the target blocks are already in the initial configuration and later when they are not.

If the blocks in the initial configuration are the same as in the target configuration, that is, if for every subtree of the initial configuration there is a subtree in the target configuration with the same blocks (not considering the arrows), we have a different permutation of ages between the two. What we need to do then is obtain the correct permutation of the ages by accessing a trace of blocks. This can be done by using only the blocks in the configuration as we see now. We recall that, from the case above, when $f p=A$, the initial configuration has a victim block in every leaf. Therefore, there exists a trace using only at most $A$ blocks that reaches a configuration for every permutation of the arrows.

If the blocks in the initial configuration are not the same as in the target configuration, or if they are in wrong subtrees, we need to evict precise blocks 
from the tree and load them back in a different leaf in order to reach the target configuration. Again, since by only using blocks in the cache we can obtain any permutation of arrows, we can obtain a trace of blocks that points the arrows to a specific block, evicts it and then modifies the arrows to load it back in a different leaf. Once the target blocks are in their corresponding leaves, we shift the arrows as before to obtain the target configuration.

\section{Results for Empty Caches}

The case of an empty cache is more complex to analyze. First we need to explain a special behavior of PLRU that produces extra reachable configurations which increases its absorption with respect to the other two policies.

Example 5.10. Consider a 4-way cache that starts in a configuration consisting of the attacker's blocks $\left\{x_{0}, x_{1}, x_{2}, x_{3}\right\} \subseteq B_{a}$ where we are going to access three victim blocks in a specific order, $b_{1}, b_{2}, b_{3} \in B_{v}$. For any of the three replacement policies the configuration becomes:

$$
\left[x_{0}, x_{1}, x_{2}, x_{3}\right] \underset{a}{\leadsto}\left[b_{1}, x_{0}, x_{1}, x_{2}\right] \underset{b}{\leadsto}\left[b_{2}, b_{1}, x_{0}, x_{1}\right] \underset{c}{\leadsto}\left[b_{3}, b_{2}, b_{1}, x_{0}\right],
$$

where the leftmost element of the lists has age zero and the one on the right is the oldest. Consider that we now access block $b_{2}$ again. The configurations transition to: $\left[b_{2}, c, b_{1}, x_{0}\right]$ for LRU, $\left[b_{3}, b_{2}, b_{1}, x_{0}\right]$ for FIFO and $\left[b_{2}, b_{3}, x_{0}, b_{1}\right]$ for PLRU (note the age of the last attacker's block $x_{0}$ ). The configuration obtained by PLRU is unreachable for the other two replacement policies, since they always fill up the cache consecutively from left to right. This illustrates how the information absorption for PLRU is larger than for the other policies.

The example is independent of the blocks being used but a consequence of the fact that we are accessing $k<A$ blocks. For LRU and FIFO, any trace using $k<A$ victim blocks will transform an initial configuration of the form $\left[x_{0}, x_{1}, \ldots, x_{A-1}\right]$ to a configuration of the form $\left[\ldots, \ldots, x_{0}, \ldots, x_{A-1-k}\right]$, where victim blocks are denoted by "_". In the case of PLRU this is not always the case, as the previous example shows.

Following our definition of absorption, we assume that the victim may access any trace of blocks. Then the number of reachable configurations can be determined as follows:

1. Determine the set of reachable layouts, i.e., configurations in which the victim's memory blocks are not distinguished from each other, but instead represented by the placeholder "_".

2. Determine for each layout the number of concrete configurations the layout represents, i.e., the number of ways the victim's blocks may fill its placeholders. 
This procedure can further be simplified upon by the following observation: The number of concrete configurations that a layout represents, only depends on its number of placeholders and the number of victim blocks to consider: Given $k$ placeholders and $f p \geq k$ victim's memory blocks, a layout represents exactly $\frac{f p !}{(f p-k) !}$ configurations.

Let $\Lambda_{\mathrm{P}}(k, A)$ denote the number of reachable layouts under algorithm $\mathrm{P}$, associativity $A$, with exactly $k$ placeholders. Accessing $f p$ distinct memory blocks may yield layouts with 0 to $f p$ many placeholders. Based on this notion, we obtain the following general characterization of a replacement policy's absorption:

Proposition 5.11. For any permutation-based cache algorithm $\mathrm{P}$, the absorbed information starting from an empty cache is:

$$
A b s_{\text {emp }}(\mathrm{P}, f p)=\sum_{k=0}^{\min \{f p, A\}} \Lambda_{\mathrm{P}}(k, A) \frac{f p !}{(f p-k) !} .
$$

Lemma 5.12. For LRU and FIFO, $\Lambda_{\mathrm{P}}(k, A)=1$ for any number of placeholders $k$ and associativity $A$. For PLRU, $\Lambda_{\mathrm{PLRU}}(k, A)$ is given by:

$$
\Lambda_{\mathrm{PLRU}}(k, A)=2 \cdot \sum_{i=\max \left\{1, k-\frac{A}{2}\right\}}^{\min \left\{\frac{A}{2}, k-1\right\}} \Lambda_{\mathrm{PLRU}}\left(i, \frac{A}{2}\right) \cdot \Lambda_{\mathrm{PLRU}}\left(k-i, \frac{A}{2}\right),
$$

if $1<k<A$ and $\Lambda_{\mathrm{PLRU}}(k, A)=1$ if $k \leq 1$ or $k=A$.

Proof. For LRU and FIFO, since they fill the cache placing $k$ victim's blocks in the $k$ youngest ages, there is only one possible layout for each value of $k$.

For PLRU we distinguish the three cases of $k$. If $k=1$, there is only one block which is mapped to age 0 . Repetitions of the same block do not modify the ages and so this is the only reachable layout.

If $k=A$, the configuration is completely filled with placeholders and so it is the only reachable layout.

If $1<k<A$, we use the representation of PLRU caches as trees with blocks on the leaves and arrows on the non-leaf nodes. We consider that we access any trace of blocks that ends up with exactly $k$ victim's blocks in the cache and study in which leaves these blocks can be. Note that we do not require the trace to have exactly $k$ different blocks but rather at least $k$. Extra blocks may evict other victim's blocks and still end up with $k$ blocks in the cache.

We base the proof on the behavior of the root of the tree, its arrow and its two children. We study how different traces of memory blocks s affect them and use it to explain the elements of (5.5). Since $\Lambda_{\text {PLRU }}$ is constructed recursively, applying it to a child is equivalent to considering the child as tree on its own. 
We first prove that any reachable configuration with exactly $k$ victim's memory blocks (and consequently placeholders) has at least 1 placeholder in each child and at most $A / 2$. The upper bound is trivial since it is the number of leaves in the child. For the lower bound consider the root of the tree. In the initial configuration the arrow points to one child. This child stores the first block $b_{1}$ of any trace of victim's blocks, after which the arrow shifts to the other child. After this the trace of memory blocks may have repetitions of $b_{1}$, which have no effect on the configuration, before accessing a new block $b_{2}$. Therefore, the child that does not store $b_{1}$ always stores $b_{2}$. This bounds can actually be reached by the trace $b_{1} b_{2} b_{1} b_{3} b_{1} \ldots b_{1} b_{k}$. The repetitions of $b_{1}$ make the arrow always point to the same child before accessing new blocks and so the blocks $b_{2}, b_{3}, \ldots, b_{k}$ are stored in the same child, with $b_{1}$ alone in the other.

Then, the number of layouts with $k$ placeholders depends on how many ways we can distribute them in the two children, constrained to at least 1 per child and at most $A / 2$. This corresponds to the limits of the sum in (5.5); for each distribution of placeholders, we compute $\Lambda_{\text {PLRU }}$ restricted to each child.

Finally, once a trace has stored $k$ victim's blocks in the configuration, the distribution of placeholders is fixed. However, the trace may access repetitions of the blocks stored in the cache, which does not modify the number of placeholders but affects the arrow on the root, that shifts from one child to the other. Therefore, every distribution of placeholders accounts for two layouts, which produces the duplication in (5.5).

\section{Comparison of Absorption}

Let us compare the absorption of LRU, FIFO, and PLRU based on Propositions 5.7 to 5.11 , for a cache set of associativity 4 . Similar results can be obtained for any associativity. The results depicted in Figure 5.1 can be obtained both from the formulas above or by simulation of caches. We highlight the following observations.

- For each replacement policy, the absorbed information grows monotonically with the footprint, as expected.

- The absorption for an empty initial configuration is always larger than for a filled configuration.

- For a filled initial configuration, LRU absorbs always at least as much information as the other replacement policies since every configuration is always reachable. For large enough footprints, the absorption coincides for all policies.

- For an empty initial configuration PLRU absorbs most. This is due to the fact that PLRU may leave "holes" in the configuration, see Example 5.10. 


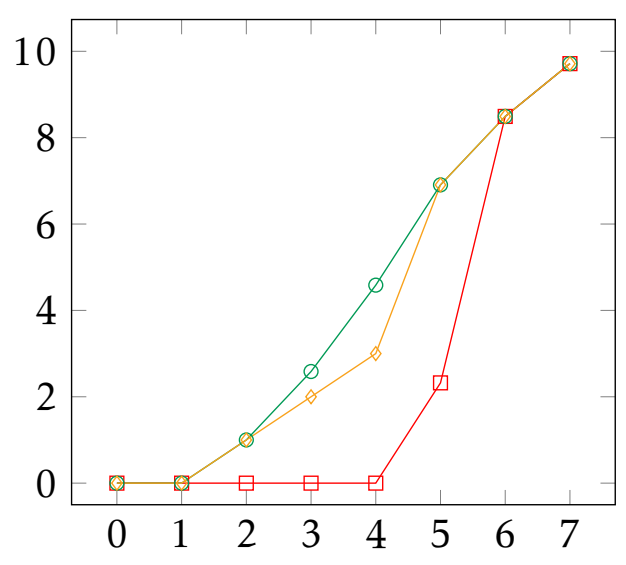

(a) Filled initial cache

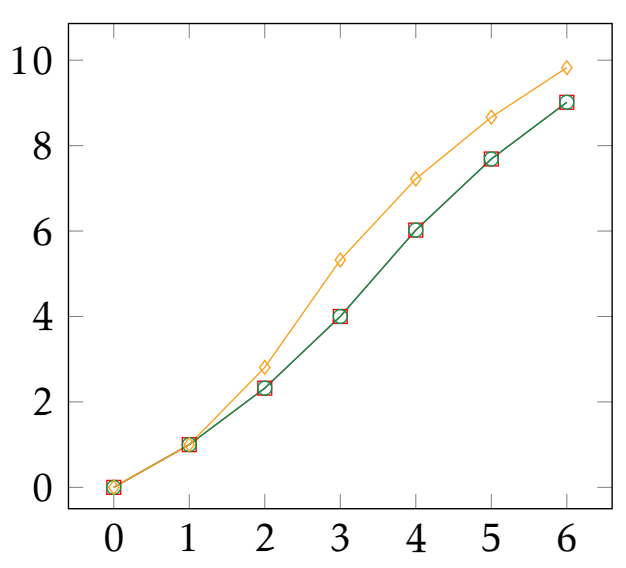

(b) Empty initial cache

$\neg-\mathrm{FIFO} \multimap-\mathrm{LRU} \multimap-\mathrm{PLRU}$

Figure 5.1: Information absorption of a 4-way cache set. Figure 5.1a depicts the case of a filled initial cache, part $5.1 \mathrm{~b}$ an empty one. In both figures, the horizontal axis depicts the footprint, i.e., the number of memory blocks used. The vertical axis depicts the absorbed information on a logarithmic scale, that is, converting the number observations into bits of information. Note that in Figure $5.1 \mathrm{~b}$, the line for LRU and FIFO coincides.

- For a filled initial cache, FIFO does not absorb any information, whenever the footprint is smaller than the associativity. This captures the intuition that preloading of sensitive data can increase security, as long as all data fits into the cache. In case it does not, the positive effect of preloading is, however, quickly undone.

\subsubsection{Information Extraction}

We use two alternative approaches for the program-independent evaluation of extraction properties cache replacement policies. The first is to rely on theoretical results that give upper bounds on the information extraction. The second is to apply the algorithm presented in Section 4.2.2 to a set of states that represent the absorbed information for a given footprint. We determine that set for each cache replacement policy by a simple fix-point computation. This algorithmic approach is more precise because it takes the absorbed information as a baseline, but it comes at the expense of higher computational cost.

\section{Extraction on Permutation-based Algorithms}

Recall that in Section 2.2 we saw that, for permutation-based cache algorithms, each cache set behaves independently of the rest. Therefore, when computing 
the information extraction on a set-associative cache, one can do it set by set, treating them as fully associative caches.

Here we derive bounds on the maximum information leakage for three permutation-based cache algorithms, on fully associative caches and for the two types of memory the attacker can use, shared and disjoint. We prove bounds for LRU and FIFO, for both shared and disjoint memory, and for PLRU for disjoint memory based on the associativity of the cache and prove that for PLRU this bound depends also on the footprint and grows with it.

We assume that, before the victim accesses the cache, it is filled with memory blocks only accessible to the attacker, that is, blocks from the set $B_{a} \backslash B_{v}$. This way, if the victim does not completely fill the cache, the attacker is able to observe it which gives more general security guaranties. This is specially useful in the case of a disjoint memory attacker.

Notation. Given a set of configurations $\widehat{G}$ we use the following shortcuts: $\widehat{G}(b)=\{\hat{g}(b) \mid \hat{g} \in \widehat{G}\}, \widehat{u p d}\left(\widehat{G}, b_{0} \ldots b_{n-1}\right)=\left\{\widehat{u p d}\left(\hat{g}, b_{0} \ldots b_{n-1}\right) \mid \hat{g} \in \widehat{G}\right\}$ and eff $(\widehat{G}, b)=\{e f f(\hat{g}, b) \mid \hat{g} \in \widehat{G}\}$.

Definition 5.13. We say that a set of configurations $\widehat{G}$ has $n \leq A$ deterministic ages if all the configurations in $\widehat{G}$ have the same $n$ youngest blocks. That is, if there exists a trace of blocks $b_{0} \ldots b_{n-1}$ such that $\widehat{G}\left(b_{i}\right)=\{i\}$ for all $i \leq n-1$.

Lemma 5.14. Let $\widehat{G}$ be a set of configurations of associativity $A$ and let $p$ be a probe. We have that $p$ is depleted if and only if $F K(p)$ has A deterministic ages.

Proof. Since $p$ is depleted we have that $|F K(p)|=1$. Then $F K(p)$ trivially has $A$ deterministic ages.

If $F K(p)$ has $A$ deterministic ages, all the blocks are mapped to the same age for every configuration of $F K(p)$ (either the deterministic ages or age $A$ ). This means that $|(F K(p))|=1$ which implies that it is unrefinable and so $p$ is depleted.

Lemma 5.15. Consider a set of configurations $\widehat{G}$ of associativity $A$ that uses either LRU or FIFO. Accessing a block mapped to a deterministic age has no effect on the number of deterministic ages.

Proof. Assume that $\widehat{G}$ has $n$ deterministic ages. Then there exists a trace of blocks $b_{0} \ldots b_{n-1}$ such that $\widehat{G}\left(b_{i}\right)=\{i\}$ for all $i \leq n-1$. Any access to a block $b_{j} \in\left\{b_{0}, \ldots, b_{n-1}\right\}$ results in a hit. For LRU, following (2.6), the new ages are:

$$
\widehat{u p d}\left(\widehat{G}, b_{j}\right)\left(b_{i}\right)= \begin{cases}\{0\} & \text { if } i=j \\ \{i+1\} & \text { if } i<j \\ \{i\} & \text { if } i>j\end{cases}
$$

so the blocks $a_{0} \ldots a_{n-1}$ are still mapped to the first $n$ ages for all configurations which results in $n$ deterministic ages. For FIFO, since hits do not reorder blocks (2.5), the conclusion is the same. 
Lemma 5.16. Consider a set of configurations $\widehat{G}$ of associativity $A$ and a trace of $n$ blocks $b_{0} \ldots b_{n-1}$ with $b_{i} \neq b_{j}$ for any $i \neq j$.

If the cache uses LRU, for any $i \leq n$, we have that $\widehat{u p d}\left(\widehat{G}, b_{0} \ldots b_{n}\right)\left(b_{i}\right)=\{n-i\}$.

The same result holds for FIFO if each of the accesses results in a miss, i.e., $\widehat{u p d}\left(\widehat{G}, b_{0} \ldots b_{i-1}\right)\left(b_{i}\right)=\{A\}$ for all $i \leq n-1$.

Proof. We proceed by induction.

Induction base. When $n=0, \widehat{u p d}\left(\widehat{G}, b_{0}\right)\left(b_{0}\right)=0$; LRU places the block in the beginning for both hits (2.6) and misses (2.4) and FIFO does it for misses (2.4), by assumption this is the case for all blocks.

Induction step. We assume the hypothesis is true for $n$ and access $b_{n+1}$. This block is distinct from the previous ones so, by the induction hypothesis, we have $\widehat{u p d}\left(\hat{g}, b_{0} \ldots b_{n}\right)\left(b_{n+1}\right)>n$ for any $g \in \widehat{G}$, i.e. $b_{n+1}$ is older than the previous. For FIFO its age is actually $\widehat{u p d}\left(\widehat{G}, b_{0} \ldots b_{n}\right)\left(b_{n+1}\right)=\{A\}$. Then $b_{n+1}$ is placed at age zero, $\widehat{u p d}\left(\widehat{G}, b_{0} \ldots b_{n+1}\right)\left(b_{n+1}\right)=\{0\}$, and the others increase their ages by one, $\widehat{u p d}\left(\hat{g}, b_{0} \ldots b_{n+1}\right)\left(b_{i}\right)=\widehat{u p d}\left(\hat{g}, b_{0} \ldots b_{n}\right)\left(b_{i}\right)+1=n+1-i$ for all $\hat{g} \in \widehat{G}$ and $i \leq n$.

Assume now that we have a set of configurations $\widehat{G}$ and we access a trace of memory blocks $b_{0} \ldots b_{k-1}$ with the requirements given in Lemma 5.16. Following this Lemma, $\widehat{u p d}\left(\widehat{G}, b_{0} \ldots b_{k-1}\right)$ has $k$ deterministic ages with $b_{i}^{\prime}=b_{k-1-i}$ from Definition 5.13. Now consider that we extend the trace of blocks with $b_{k} \ldots b_{m}$ with $b_{i} \in\left\{b_{0}, \ldots, b_{k-1}\right\}$ for $k \leq i \leq m$, that is, with blocks already mapped to deterministic ages. Then following Lemma $5.15 \widehat{u p d}\left(\widehat{G}, b_{0} \ldots b_{m}\right)$ still has $k$ deterministic ages.

If we continue to extend the trace with new blocks (provided they produce misses for FIFO) we will produce extra deterministic ages on the updated set of configurations. If we extend with blocks already mapped to deterministic ages, the number of deterministic ages is not modified.

Proposition 5.17. Consider LRU and FIFO with associativity $A$ and a shared memory attacker. The maximum information leakage on any set of configurations is bounded by $2^{A}$ for LRU and by $(A+1)$ ! for F IF 0 .

Proof. LRU. Consider any given strategy att and any probe produced by att, $p=b_{1} o_{1} \ldots b_{n} o_{n}$ with $n \geq A$ and $\left|\left\{b_{1}, \ldots, b_{n}\right\}\right|=A$. Following Lemmas 5.15 and 5.16, since $b_{1} \ldots b_{n}$ is formed with alternating sub-traces of new blocks and repetitions of them, $F K(p)=\widehat{u p d}\left(K(p), b_{1} \ldots b_{n}\right)$ has $A$ deterministic ages which, by Lemma 5.14 , means that $p$ is depleted. Then any probe is depleted w.r.t. att if it has $A$ different blocks. 
We now prove that, for any probe given by att, repetitions of blocks do not partition the knowledge sets. Given the non-depleted probe $p=b_{1} o_{1} \ldots b_{n} o_{n}$, for any value of $n$, we have that a new block $b$ produces $|\operatorname{eff}(F K(p), b)|=1$ if $b=b_{i}$ for some $i \leq n$ since $b_{i}$ is mapped to a deterministic age. For any other block, the view function is trivially bounded by 2 , $|\operatorname{eff}(F K(p), b)| \leq 2$.

Given the set of possible configurations $\widehat{G}_{v}$, the first block given by att will produce at most two knowledge sets. For each of these knowledge sets, the second block will partition them into two knowledge sets, making a total of up to four knowledge sets, unless it is a repetition, in which case there is no partition. We can partition the knowledge sets further until the probes are depleted, which happens after $A$ different blocks. Each not repeated block at most doubles the amount of knowledge sets so, after $A$ blocks the strategy produces up to $2^{A}$ knowledge sets.

FIFO. Consider any given strategy att and any probe produced by att, $p=$ $b_{1} o_{1} \ldots b_{n} o_{n}$ with $n \geq A$ and $A$ misses. Following Lemmas 5.15 and 5.16 , since $b_{1} \ldots b_{n}$ is formed with alternating sub-traces of new blocks and repetitions of them, $F K(p)$ has $A$ deterministic ages which, by Lemma 5.14, means that $p$ is depleted. Then any probe is depleted w.r.t. att if it has $A$ misses.

Consider a non-depleted probe $p=b_{1} o_{1} \ldots b_{n} o_{n}$, for any value of $n$, that we extend with a block $b$ such that eff $(F K(p), b)=\{\mathrm{H}\}$. Then $F K(p b \mathrm{H})=F K(p)$, that is, obtaining a hit for all the configurations has no effect on the partition. A block $b$ can return a hit for all the configurations in a final knowledge set in two cases:

1. The probe, starting from the last miss, is one of the form

$$
p=b_{1} \mathrm{M} b_{2} \mathrm{H} \ldots b_{n} \mathrm{H} b \mathrm{H}
$$

with $b=b_{i}$ for some $i \leq n$.

2. The block $b$ is mapped to a deterministic age.

We now have a characterization of how depleted probes of minimal length look like. First, all depleted probes have $A$ misses but may have a different number of hits between each miss. Second, in order for the probes to be of minimal length, their blocks do not return a hit for all the configurations in the current final knowledge set.

Since new misses introduce new deterministic ages, the maximum number of consecutive non-repetitive hits is reduced by one with each miss. Before any miss, att can produce up to $A$ hits with these restrictions, which gives up to $A+1$ different probes. After the first miss, att extends each probe with up to $A-1$ consecutive hits which makes up to $(A+1) A$ different probes. In the end, any attack strategy can produce up to $(A+1)$ ! depleted probes of minimal length and the same amount of knowledge sets. 
Proposition 5.18. Consider PLRU with associativity $A \geq 4^{1}$ and a shared memory attacker. Let $\mu(f p)$ be the maximum information leakage obtained with a given footprint $f p \geq A$. It holds that $\mu(f p+1) \geq \mu(f p)+1$.

Proof. Let att be an attack strategy that obtains $\mu(f p)$ given a set of possible configurations. We are going to prove that one empty knowledge set from $R_{\text {att }}$ when using $f p$ blocks is non-empty given an extra memory block i.e., when using $f p+1$ blocks.

Consider a probe where the first $A-1$ observations are misses and the corresponding final knowledge set $\widehat{G}_{1}$; all the configurations in this set have the same blocks mapped to the younger ages and an unknown one in age $A-1$ that we call $b^{\prime}$. Consider the set of yet unused victim's blocks $B_{v}^{\prime} \subset B_{v}, b^{\prime} \in B_{v}^{\prime}$; any access to a block from that set evicts $b^{\prime}$ in the case of a miss. But before we access a block from $B_{v}^{\prime}$ we access a previously used block and update the configurations so that $b^{\prime}$ is mapped to age $A-2$. We use the block in age 1 to modify the age of $b^{\prime}$ since $\Pi_{\mathrm{PLRU}}(1, A-1)=2 \cdot \Pi_{\mathrm{PLRU}}(0, A / 2-1)=2 \cdot(A / 2-1)=A-2$, see (2.7). In order to do this, the associativity must be at least 4 so that $A-1>1$.

After this, a new access to a block from $B_{v}^{\prime}$ does not evict $b^{\prime}$ in the case of a miss. Therefore, the block partitions $\widehat{G}_{1}$ into a knowledge set with $b^{\prime}$ and another knowledge set without $b^{\prime}$ but an unknown block in age $A-1$. Repeating this process of placing the block from age $A-1$ in age $A-2$ and later accessing a new block allows to partition the knowledge set that returned a miss, without evicting an unknown block in the case of a miss, and therefore maximize the number of knowledge sets.

There is an attack strategy att that follows this process in order to obtain $\mu(f p)$. Then the knowledge set of the probe where all the observations are misses is empty. Now suppose that we have an extra memory block. Then, the knowledge set of the probe where all the observations are misses is not empty as it contains configurations with the extra block in age $A-1$. This way we have increased the maximum information leakage by one.

In the case of associativity four for PLRU the maximum information leakage is increased by eight with every new memory block, this can be seen in Figures $5.2 \mathrm{e}$ and 5.2f. This result also implies that the maximum information leakage for PLRU is unbounded.

Proposition 5.19. Consider FIFO and LRU with associativity $A$, and a disjoint memory attacker. The maximum information leakage on any set of configurations is bounded by $A+1$.

Proof. We base the proof on the configuration of the cache before the victim accesses it. There are $A$ attacker's blocks $x_{0}, \ldots, x_{A-1}$ such that $c\left(x_{i}\right)=i$.

\footnotetext{
${ }^{1}$ Note that for associativity 2, PLRU and LRU coincide.
} 
Now we make use of the fact that for both, $M_{\mathrm{FIF} 0}$ and $M_{\mathrm{LRU}}$, for any block $b^{\prime}, \hat{g}(b)=\widehat{u p d}\left(\hat{g}, b^{\prime}\right)(b)$ if $\hat{g}(b)>\hat{g}\left(b^{\prime}\right)$, see (2.5) and (2.6). This means that, when the attacker accesses one block he does not modify the ages of older ones. This is a way to probe the cache without evicting any blocks.

Assume the attacker accesses block $x_{0}$; he gets a hit or a miss and partitions $\widehat{G}_{v}$ accordingly. The knowledge set that returned a miss has configurations with zero attacker's blocks, all accesses to attacker's blocks return the same output so it can not be partitioned further. The other knowledge set has at least one attacker's block and, following the property configurationd above, the older blocks, i.e. $x_{1}, x_{2}$, etc, have not been evicted. Now the attacker accesses block $x_{1}$ and partitions the set into the configurations with exactly one attacker's block $\left(x_{0}\right)$ and the ones with at least two.

Following this trace $x_{0}, x_{1}, \ldots$, every memory block singles out one unrefinable knowledge set but does not affect future accesses. In the end the attacker produces up to $A+1$ knowledge sets.

Proposition 5.20. Consider PLRU with associativity A, footprint fp, and a disjoint memory attacker. The maximum information leakage is bounded by $\sum_{k=0}^{f p} \Lambda_{\mathrm{PLRU}}(k, A)$ where $\Lambda_{\mathrm{PLRU}}(k, A)$ is defined as in (5.5).

Proof. The information extraction is intuitively bounded by the number of layouts of attacker's and victim's blocks that a disjoint-memory attacker can distinguish. For each value of $k \in[0, f p], \Lambda_{\mathrm{PLRU}}(k, A)$ gives the number of possible layouts using $k$ victim's blocks, leaving $A-k$ attacker's blocks. Therefore, by summing all of them up to the used footprint, we obtain the total number of layouts. Note that this bound may not be tight.

\section{Experimental Results}

We now obtain experimental values for the information extraction by applying Algorithm 1 to a worst-case set of reachable configurations obtained by fix-point computation. In this case, we consider a single 4-way cache set. Figure 5.2 depicts our data. We highlight the following results:

- For shared-memory attackers, FIFO and LRU reach the bound on the maximum information leakage given in Proposition 5.17, which is independent of the footprint, see Figures 5.2a to 5.2d. In contrast, with PLRU the number of knowledge sets increases with the footprint as predicted by Proposition 5.18, see Figures 5.2e to 5.2f.

- For disjoint-memory attackers and a filled initial state we always obtain zero leakage. For PLRU and a footprint of 2 or 3 some cache lines remain unoccupied. As before, these unoccupied lines trigger additional observations, which explain the bump in Figure 5.2e. 


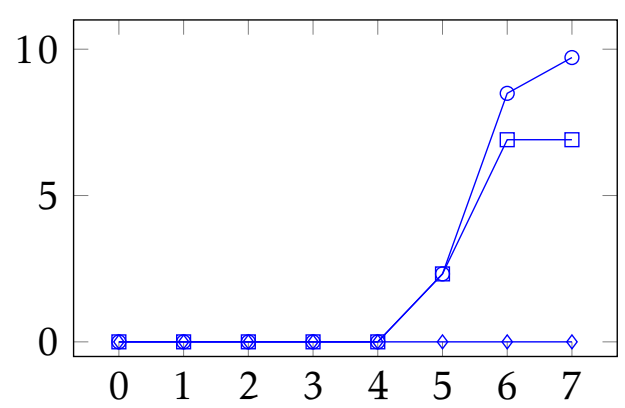

(a) Filled cache using FIFO.

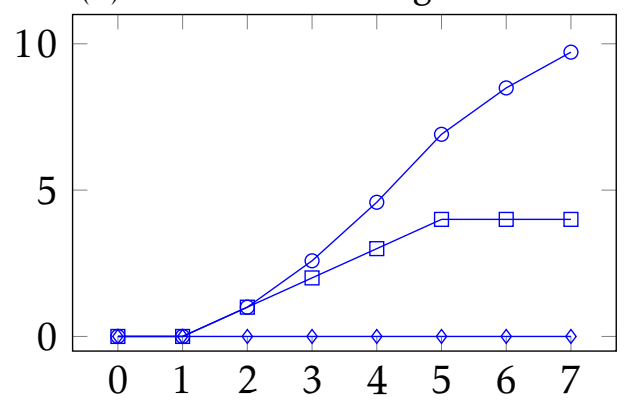

(c) Filled cache using LRU.

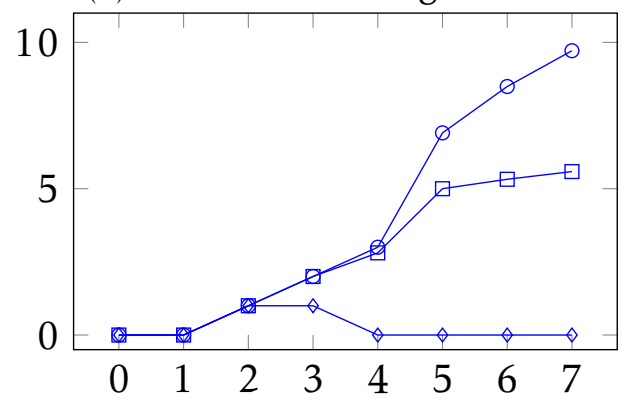

(e) Filled cache using PLRU.

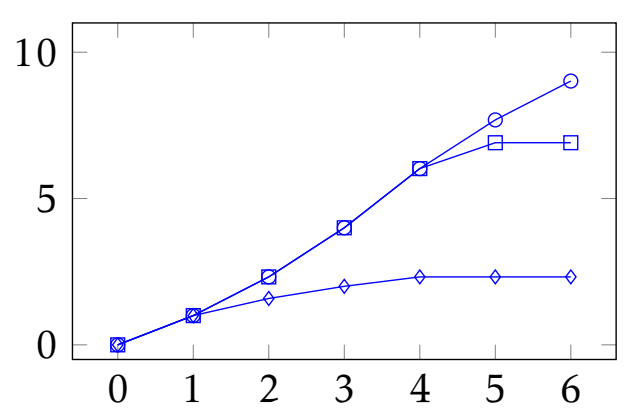

(b) Empty cache using FIFO.

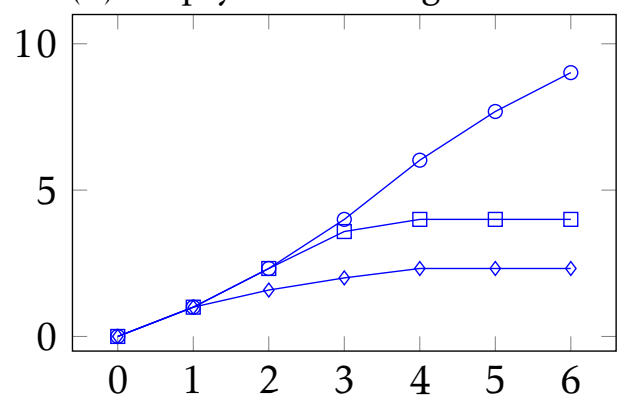

(d) Empty cache using LRU.

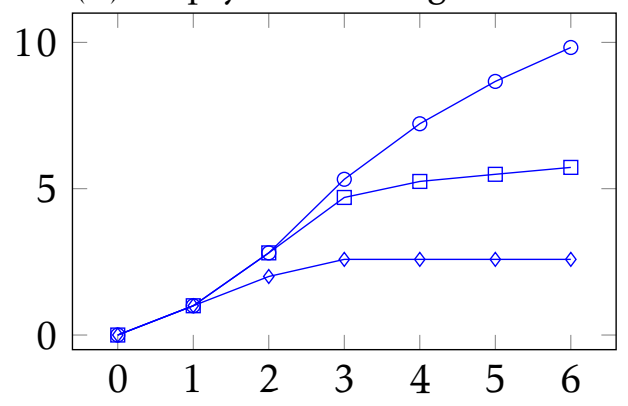

(f) Empty cache using PLRU.

$$
\multimap \text { Absorption } \square \text { Extraction (Shared) } \rightarrow \text { Extraction (Disjoint) }
$$

Figure 5.2: Information extraction of different replacement policies on a 4-way cache set. Figures 5.2a, 5.2c and 5.2e depict the case of a filled initial cache, $5.2 \mathrm{~b}, 5.2 \mathrm{~d}$ and $5.2 \mathrm{f}$ an empty one. In all figures, the horizontal axis depicts the footprint, i.e., the number of memory blocks used. The vertical axis depicts the extracted information on a logarithmic scale, that is, converting the number observations into bits of information. 
- We observe that FIFO exhibits the smallest difference between absorption and extraction among all policies, i.e. once absorbed, it is comparably easy to extract information from the cache, see Figures 5.2a to 5.2b. This is because FIFO does not reorder blocks upon hits, which makes systematic search for the cache state easier.

\subsubsection{Set-Associative Caches}

In this section we extend our results for information absorption and extraction to set-associative caches. Set-associative caches consist of several cache sets of equal associativity, i. e. each cache set has the same number of cache lines. Each memory block can only be cached in one cache set.

Ideally, in set-associative caches, the memory blocks are distributed equally among the sets. This may not be the case for actual programs as we will see later but serves as a basis to study how the leakage of the cache is affected when not all the memory blocks compete for the same cache set.

Note that the leakage for the time and trace-based attacker is not affected by the number of sets in the cache, as the effect function (2.2) only depends on the accessed memory block being cached or not, not on the distribution of the cache lines. For this reason, in this Section we only focus on the access-based attacker.

The information absorption and extraction on a single cache set depends on the footprint, the smaller the footprint, the smaller the absorption and similarly the extraction. Then, intuitively we could think that, given a program with a fixed footprint, having all the memory blocks in one cache set should produce the biggest absorption and extraction and having each memory block alone in a cache set should produce the smallest absorption and extraction. We will see that this is not necessarily the case as the effect of several cache sets sometimes increases the overall leakage even though the leakage per cache set is reduced.

Note also that we are assuming that the cache can have any number of cache sets. In reality this is usually not the case: caches have a number of cache sets which is a power of two which facilitates implementing the set function [HP11]. Nonetheless, we can theoretically compute the leakage for any number of cache sets.

\section{Information Absorption}

As we saw when dealing with fully associative caches, the bigger the footprint the bigger the information absorption. Therefore, if we distribute the memory blocks into independent sets, we reduce the footprint per set and so the absorption per set. However, the multiplicative effect of several cache sets may counter the reduction of absorption per set. 


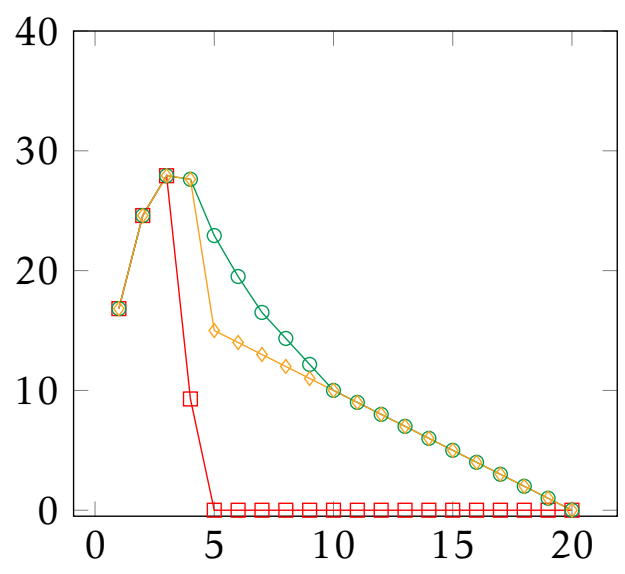

(a) Filled initial cache

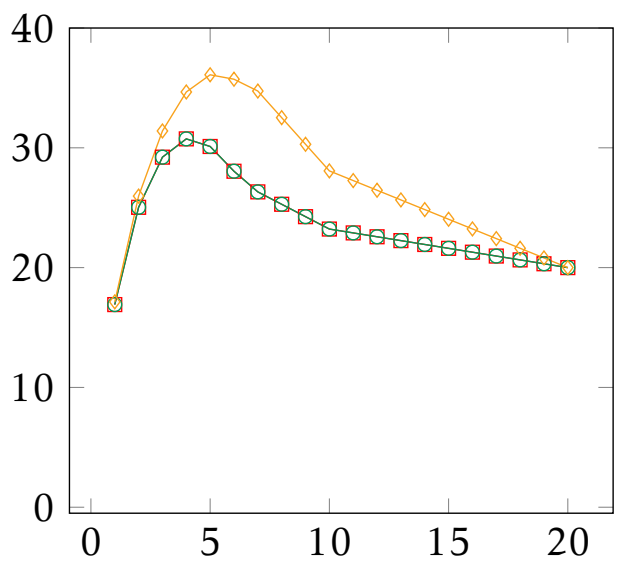

(b) Empty initial cache $\square$ FIFO $\odot$ LRU $\diamond-$ PLRU

Figure 5.3: Information absorption of different replacement policies on a 4way cache with 20 memory blocks of footprint and an increasing number of cache sets. Figure 5.3a depicts the case of a filled initial cache, part 5.3b an empty one. In all figures, the horizontal axis depicts the number of cache sets. The vertical axis depicts the extracted information on a logarithmic scale, that is, converting the number observations into bits of information. Note that in Figure 5.3b, the line for LRU and FIFO coincides.

Figure 5.3 depicts the information absorption on a cache with associativity 4, 20 memory blocks of footprint and a number of cache sets that varies from 1 to 20 , that is, from one cache set where the 20 memory blocks are mapped to, to 20 cache sets, each one with one memory blocks to store. We highlight the following conclusions:

- Right away we see that the largest absorption value does not occur when all the memory blocks are mapped to the same cache set but when each cache set stores about as many memory blocks as the associativity of the cache:

- Six or seven memory blocks for FIFO on a filled cache (three cache sets), Figure 5.3a.

- Five memory blocks for LRU and PLRU on a filled cache (four cache sets), Figure 5.3a.

- Five memory blocks for LRU and FIFO on an empty cache (four cache sets), Figure 5.3b.

- Four memory blocks for PLRU on an empty cache (five cache sets), Figure 5.3b. 
This happens because the overall absorption of the cache is obtained as the product of the absorptions of each cache set. Although when we increase the number of cache sets the footprint per set diminishes, at first the multiplication makes the overall absorption grow faster than the reduction of absorption per set. Once each cache set holds less or about as many memory blocks as its associativity, the absorption per set is reduced enough so that the multiplication does not increase the overall absorption.

- Moreover, for FIFO and PLRU on a filled cache, Figure 5.3a, the special cases when the footprint is bellow the associativity, Propositions 5.8 and 5.9 , account for the sudden drop in absorption.

- In the case of a filled cache, Figure 5.3b, the absorption goes to zero as we increase the number of cache sets. Since the footprint is fixed, as we increase the number of cache sets, each cache set has less memory block to store. Once there is one block per set, there is only one possible configuration of the cache (the memory block mapped to age zero) which translates into no leakage. For an empty cache, Figure 5.3b, when there is one memory block per set, each cache set can have two configurations (the block cached or not). This means one bit of leakage per set and so twenty bits when we combine all the cache sets.

- In the case of an empty cache, Figure 5.3b, we actually have less absorption with one cache set than with twenty. This effect occurs when the footprint is large enough and the difference actually grows as we increase the footprint, see Figure 5.5a.

\section{Information Extraction}

Similarly as with information absorption, the bigger the footprint the bigger the information extraction. Therefore, if we distribute the memory blocks into independent sets, we reduce the footprint per set and so the extraction per set. However, the multiplicative effect of several cache sets counters the reduction of extraction per set.

Figure 5.4 depicts the information extraction for each replacement policy alongside with the information absorption. We now mention some of the conclusions we obtain:

- For the shared memory attacker we see that the case of largest information extraction occurs when having several cache sets but not too many so that the footprint per set is too small. These maximum values more or less coincide with the maximum values on information absorption. 


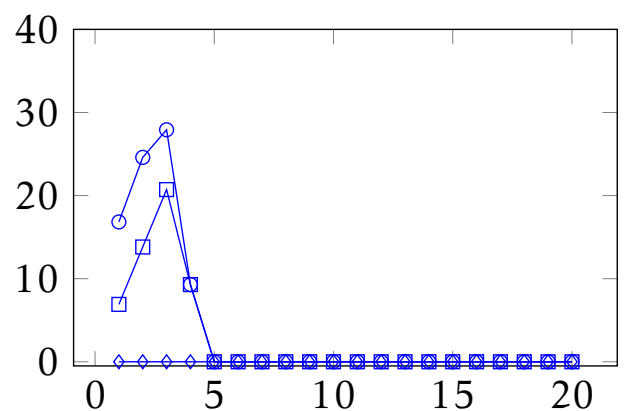

(a) Filled cache using FIFO.

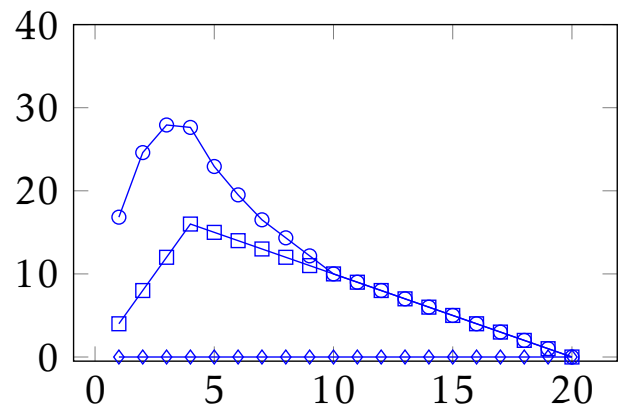

(c) Filled cache using LRU.

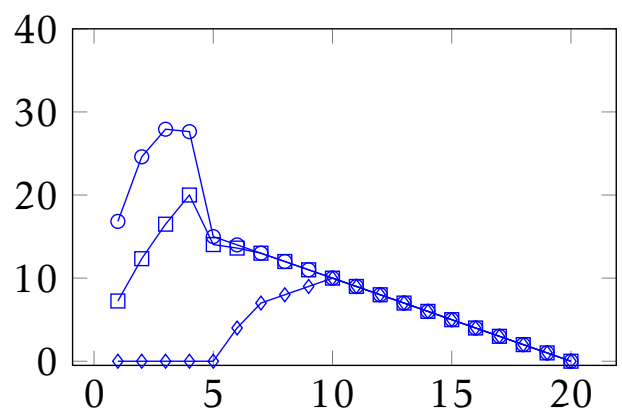

(e) Filled cache using PLRU.

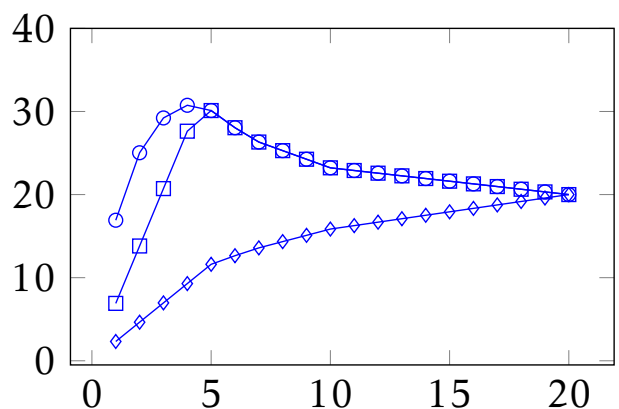

(b) Empty cache using FIFO.

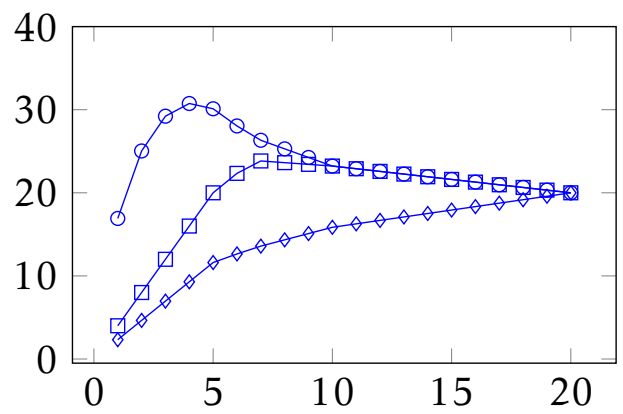

(d) Empty cache using LRU.

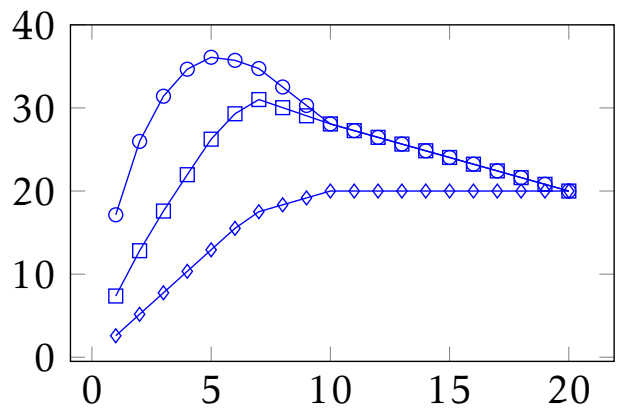

(f) Empty cache using PLRU.

$\multimap$ Absorption $\square-$ Extraction (Shared) $\multimap-$ Extraction (Disjoint)

Figure 5.4: Information extraction of different replacement policies on a 4way cache with 20 memory blocks of footprint and an increasing number of cache sets. Figures 5.2a, 5.2c and 5.2e depict the case of a filled initial cache, $5.2 \mathrm{~b}, 5.2 \mathrm{~d}$ and $5.2 \mathrm{f}$ an empty one. In all figures, the horizontal axis depicts the number of cache sets. The vertical axis depicts the extracted information on a logarithmic scale, that is, converting the number observations into bits of information. 
- This is intuitively clear for LRU and FIFO as they have an upper bound on the information extraction per set that is reached whenever the set of reachable configurations is large enough, Proposition 5.17. Then, as long as the absorption per set is large enough, the extraction grows with every new set.

- For PLRU, although the extraction depends on the footprint, Proposition 5.18, for associativity four it is a linear dependency, see Figures $5.2 \mathrm{e}$ and $5.2 \mathrm{f}$. Therefore, when we increase the number of sets and reduce the footprint, the multiplication takes over the linear reduction of extraction per set.

- Since the information extraction is upper bounded by the information absorption, once the absorption decreases, it takes the information extraction with it.

- For the disjoint memory attacker we see that the information extraction on an empty cache grows as we increase the number of sets. This is again due to the fact that extraction on a disjoint memory attacker is upper bounded by a constant independent of the footprint, Propositions 5.19 and 5.20, and increasing the number of sets makes the extraction grow.

- For the disjoint memory attacker on a filled cache we have zero leakage for FIFO and LRU, as expected since in Figures 5.2a and 5.2c the extraction is zero for all footprints, but for PLRU the extraction grows when having more than five cache sets. This is due to the small bump for PLRU when the cache set has footprint two or three, Figure 5.2e. Since the overall footprint is twenty memory blocks, when having more than five cache sets, some of them have only two or three memory blocks to store and so produce one bit of leakage. Because of this, as we increase the number of sets, the extraction grows.

- For both types of memory attackers and when starting on an empty configuration, the extraction when having all the memory blocks in one cache set is smaller than when having one memory block per cache set. As with the information absorption, this occurs if the footprint is large enough and the difference grows with the footprint, see Figures 5.5b and $5.5 c$. 


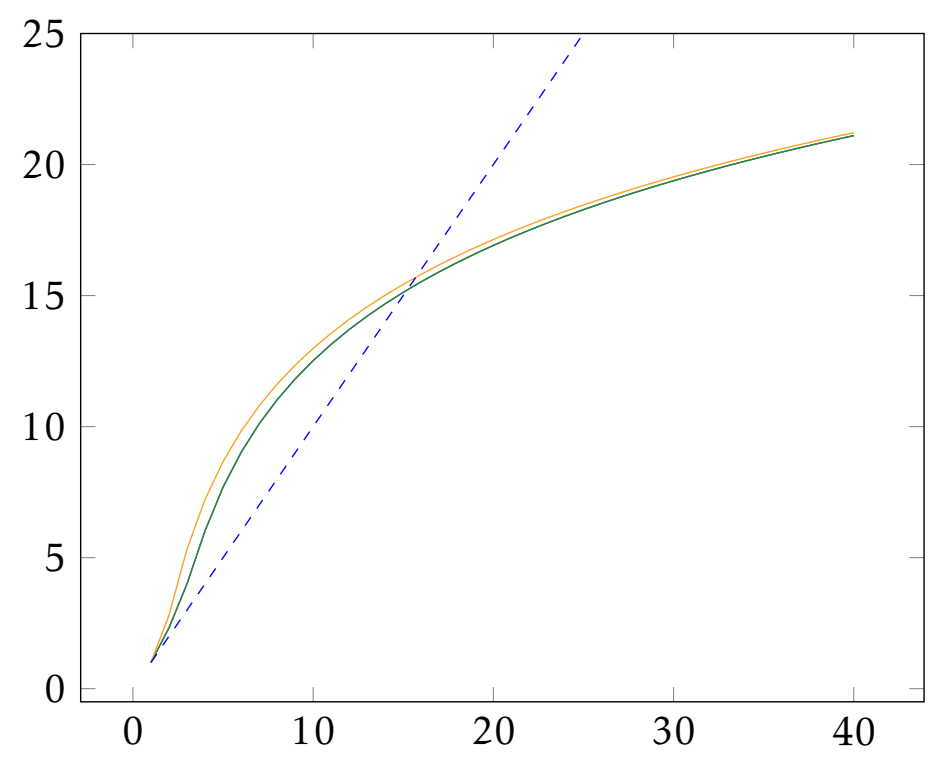

(a) Absorption

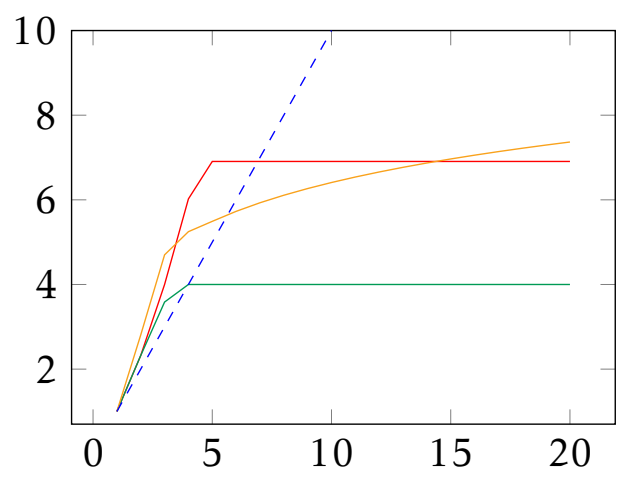

(b) Extraction (Shared)

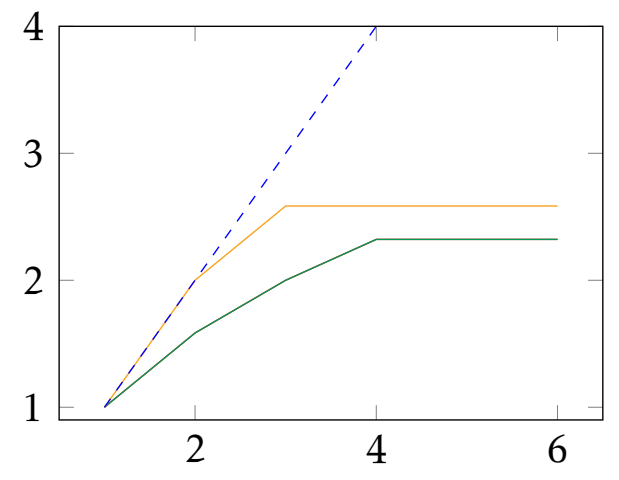

(c) Extraction (Disjoint)

- FIFO (one set)__ LRU (one set)__ PLRU (one set) - - - several sets

Figure 5.5: Difference in information absorption and extraction between one cache set and as many cache sets as memory blocks. Here we consider a 4-way cache starting on an empty configuration. Solid lines represent the leakage of one single cache set that stores all the memory blocks (one line per cache algorithm) and the dashed line represents the absorption of as many cache sets as memory blocks, i.e. one block per cache set (the same line for all algorithms). In all figures, the horizontal axis depicts the footprint. The vertical axis depicts the leakage on a logarithmic scale, that is, converting the number observations into bits of information. Note that in Figures 5.5a and 5.5c, the line for LRU and FIFO coincides. For small footprints, several cache sets produce less or equal leakage than one cache set but, when the footprint is large enough, having all the memory blocks in one cache set produces less leakage than having each one alone in a cache set. 


\section{CHAPTER 6}

\section{CACHEAUDIT WITH INFORMATION EXTRACTION}

CacheAudit is an static analyzer that, for the cache algorithms LRU, FIFO and PLRU computes an over approximation of the number of possible observations a time, trace or access-based attacker can obtain. Specifically for the access-based attacker, CacheAudit computes an over approximation of the set of reachable configurations of the cache and gives its size as a security guaranty. This basically means that CahceAudit computes the information absorption as a security guaranty.

We now use Algorithm 1 for computing the information that can be extracted from the cache configuration with respect to a specific program. For this, we use as a basis the set of reachable configurations output by the CacheAudit static analyzer, when run on an implementation of AES 256, Sosemanuk $\left[\mathrm{BBC}^{+} 08\right]$ and $\mathrm{HC}-128$ [Wu08]. In this example we use a cache consisting of several independent cache sets of associativity 4, blocks of 64 bytes and overall sizes of 4, 8, 16 and $32 \mathrm{~KB}$. For the case of AES-256, we consider two cases, one that starts from a filled cache and one that starts from an empty cache. The implementation of Sosemanuk and HC-128 only allow for an initial empty cache.

Since we know the amount of memory each implementation uses and the size of the cache lines we know how many memory blocks the cache has to store when running these three programs. Also, by having the line size, the associativity and the size of the cache, we conclude the number of cache sets. These are 16, 32, 64 and 128 cache sets for the cache sizes 4, 8, 16 and $32 \mathrm{~KB}$ respectively. With this data we compute the corresponding upper bounds for the information absorption and extraction. That is, we assume the memory blocks are evenly distributed and apply the results obtained in Figure 5.4. 
However, the counting procedure of CacheAudit has some limitations and, in some cases, it produces a set of reachable configurations larger than the one the cache can actually reach. We first address the counting procedure of CacheAudit and point to the situations where it over approximates the set of reachable configurations. Later we will analyze the three cryptographic algorithms and explain the differences between the upper bounds and the computed values.

\subsection{CacheAudit's Over Approximations}

The way CacheAudit computes the set of reachable configurations introduce some over approximations that, in some cases, lead to large differences with respect to how the set of reachable configurations should be. We now explain this procedure and point to situations where the approximation is over pessimistic.

CacheAudit analyzes permutation-based cache algorithms, see Section 2.2. It computes the set of reachable configurations using the map from memory blocks to ages. When CacheAudit analyzes a program, it obtains all the possible ages each memory block can be mapped to. With that, CacheAudit computes all the possible configurations where each memory block is mapped to one of its valid ages. For example, on a 4-way cache, if one of the cache sets has two memory blocks and both of them can be mapped to ages 0 and 4 , there exist two reachable configurations, each one having one memory block cached in the first age and the other not cached.

This introduces invalid configurations in some cases. Consider a 4-way cache using FIFO starting on a filled configuration of one cache set, and a set of five memory blocks. By looking at the upper bounds on absorption, Proposition 5.8, we know that there are five reachable configurations. However, for every memory block $b$ and age $a$, there exists one of those five configurations where block $b$ is mapped to age $a$. Since CacheAudit obtains the possible ages, it gives as reachable configurations all combinations of those five memory blocks, including the empty configuration even though the cache started filled.

A similar thing happens with PLRU when having less blocks than the associativity, Proposition 5.9. For LRU the effect is less prominent since this cache algorithm does not have cases where the information absorption is less than all possible configurations. However, in the case of a filled cache, CacheAudit gives as valid empty configurations that are not reachable from filled ones.

This effects only occur when considering an implementation that starts on a filled configuration. Whenever the cache starts on an empty configuration, CacheAudit correctly computes the set of reachable configurations. This is because of the absence of special cases for information absorption when using 
an empty cache.

\subsection{Upper Bounds for AES}

In this Section we analyze the security of AES-256 using CacheAudit, compare it to the upper bounds when using worst-case analysis and show that the difference in leakage when using both methods does not differ too much.

Figure 6.1 depicts the upper bounds of the leakage alongside with the ones computed by CacheAudit when analyzing AES 256 starting on both a filled cache and an empty one. Here we highlight the following results.

- We obtain the bounds on the information absorption and extraction corresponding to using the CacheAudit static analyzer. The difference between the information absorption and the information extraction corresponds to the precision gained by the development of the extraction Algorithm 1. This gain is generally higher when sets contain several memory blocks, and reaches up to 50 bits for LRU on a $4 \mathrm{~K}$ cache with empty initial state and a shared memory attacker, see Figure 6.1d. That is, our extraction algorithm is a simple but powerful replacement for the model counting algorithms in CacheAudit.

- We see that the difference from the upper bound to the computed value differs very little for the empty cache case and a lot for some cases on a filled cache.

- In the case of the empty cache, the difference is due to variations in the distribution of memory blocks among the cache sets. Despite the differences, the behavior of the leakage as we increase the size of the cache is similar in both the upper bounds and the computed values.

- In the case of the filled cache, the differences are too big to be a problem of the distribution of memory blocks. The issue here is the counting procedure of CacheAudit.

As we explained in Section 6.1, CacheAudit does not consider the special cases of FIFO and PLRU where, although every memory block can be mapped to any age, some configurations are still unreachable, Propositions 5.8 and 5.9. Instead, CacheAudit considers as reachable every configuration where the memory blocks are mapped to valid ages, which produces big differences in absorption and, since the set of reachable configuration is different, also in extraction. This does not apply to LRU since, for this algorithm, every configuration is reachable. 


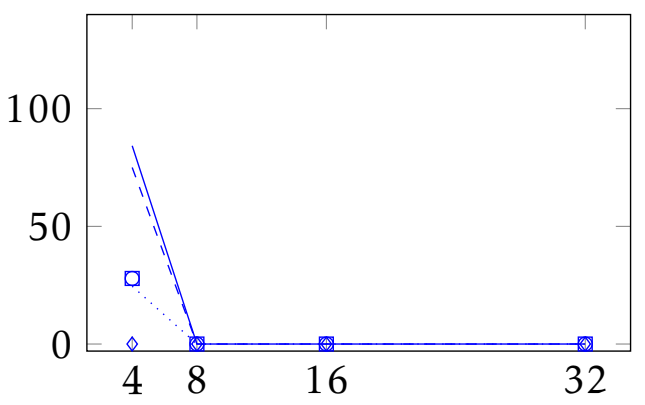

(a) Filled cache using FIFO.

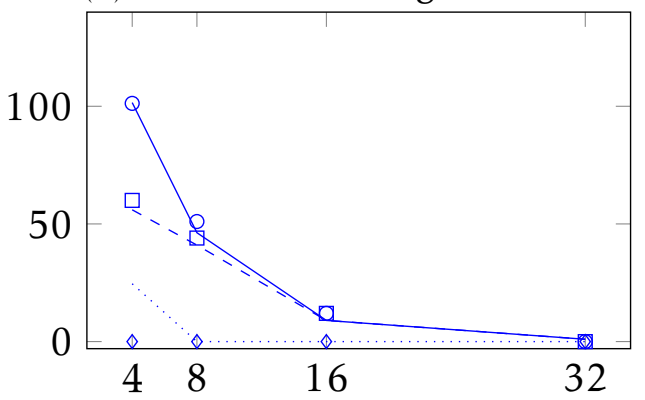

(c) Filled cache using LRU.

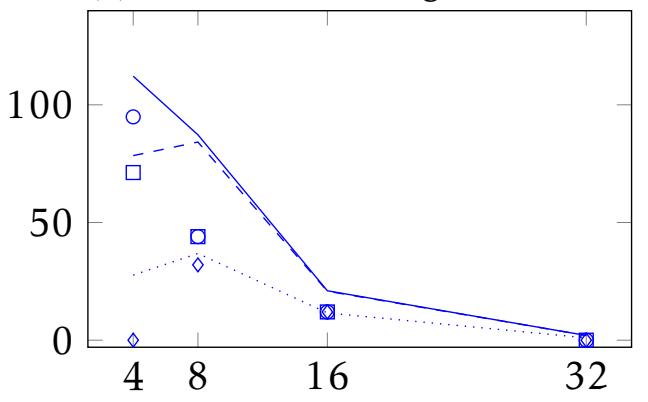

(e) Filled cache using PLRU.

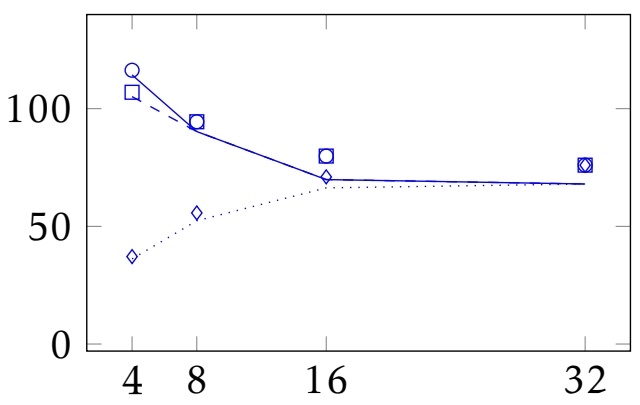

(b) Empty cache using FIFO.

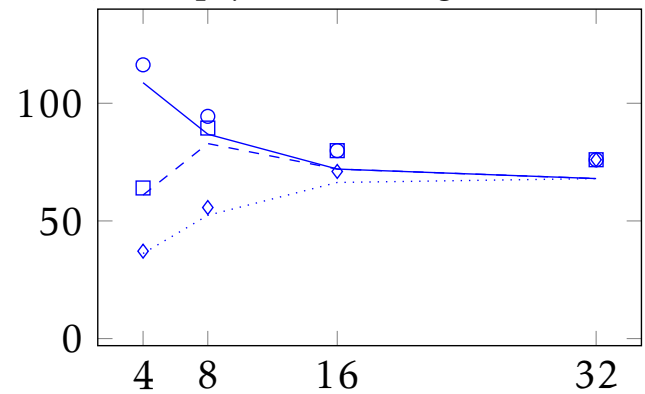

(d) Empty cache using LRU.

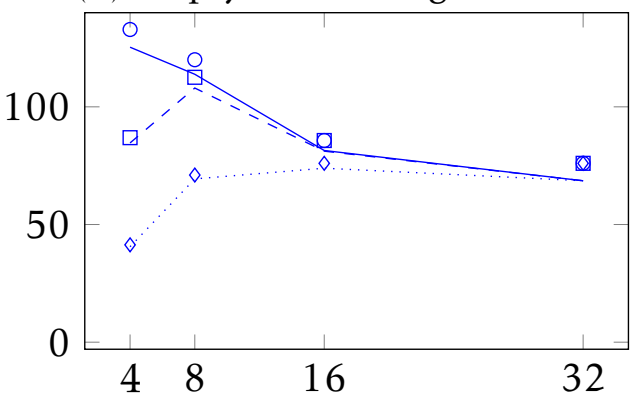

(f) Empty cache using PLRU.

\begin{tabular}{|lcc|}
\hline$\circ \quad$ Upper bound on Absorption & Computed Absorption \\
$\square$ Upper bound on Extraction (Shared) & -- Computed Extraction (Shared) \\
$\diamond$ Upper bound Extraction (Disjoint) & $\cdots$....... Computed Extraction (Disjoint)
\end{tabular}

Figure 6.1: Information absorption and extraction (in bits) for the AES-256 execution on a 4-way cache, for filled and empty initial cache states. Figures 6.1a, 6.1c and 6.1e depict the case of a filled initial cache, 6.1b, 6.1d and $6.1 \mathrm{f}$ an empty one. The horizontal axis depicts the size of the cache in $\mathrm{KB}$, the vertical axis depicts the extracted information in logarithmic scale, that is, converting the number observations into bits of information. Points represent the upper bounds on the leakage, assuming an even distribution of the memory blocks and considering the worst-case scenario. Lines represent the values computed by CacheAudit. 
- In the case of a disjoint memory attacker with filled cache and cache size of $4 \mathrm{~KB}$, the upper bound for the information extraction is zero whereas CacheAudit computes a positive value. Since all the cache sets store at least as memory blocks as the associativity, all the possible configurations have four victims blocks in them which produces the upper bound of no extraction with disjoint memory. However, for cache sets with strictly more memory blocks than the associativity, CacheAudit also considers as reachable configurations that are not completely filled, which allows for a non-zero information extraction under disjoint memory.

Except for the aforementioned counting procedure of CacheAudit, the computed values for the leakage are close to the upper bounds. This shows that the leakage of AES does not differ too much from the leakage obtained by worst-case scenario analysis, which implies that one can rely on the upper bounds when considering the security of AES. In the next Section we will see that other cryptographic algorithms have very large differences between the upper bounds and the computed values of the leakage and in that case, an analysis gives better security guaranties.

- We see a decreasing shape in the information absorption as we increase the size of the cache which corresponds to the behaviors observed in Figure 5.4 .

- For the information extraction with disjoint memory and a filled cache we see that the extraction for FIFO and LRU is zero (except for the differences in counting of CacheAudit) and PLRU has non-zero leakage due to the bump shown in Figure 6.1e.

- For the information extraction with disjoint memory and an empty cache we see that the extraction for all cache algorithms grows with the size of the cache (i.e. the number of cache sets) as shown in Figures 6.1b, 6.1d and $6.1 \mathrm{f}$.

- For the information extraction with shared memory we see that some cache algorithms present a bump, Figures $6.1 \mathrm{~d}$ and $6.1 \mathrm{f}$, and others do not, Figures 6.1a, 6.1c and 6.1b, as we increase the cache size and reduce the footprint per set. Their presence or absence is due to the fact that the point of maximum information extraction with shared memory occurs for a different footprint per set depending on the cache algorithm and whether the cache is initially filled or empty, see Figure 5.4. 

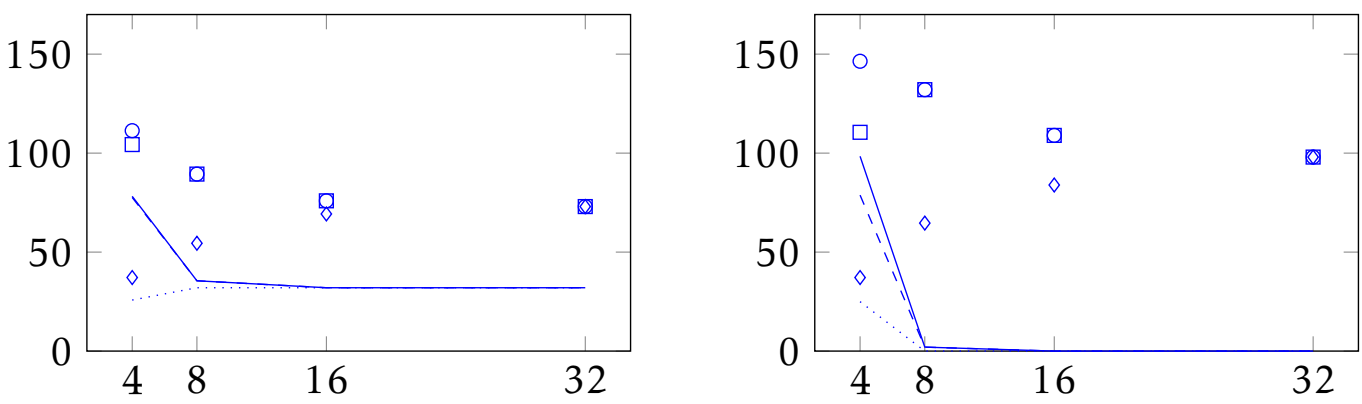

(a) Sosemanuk using FIFO.

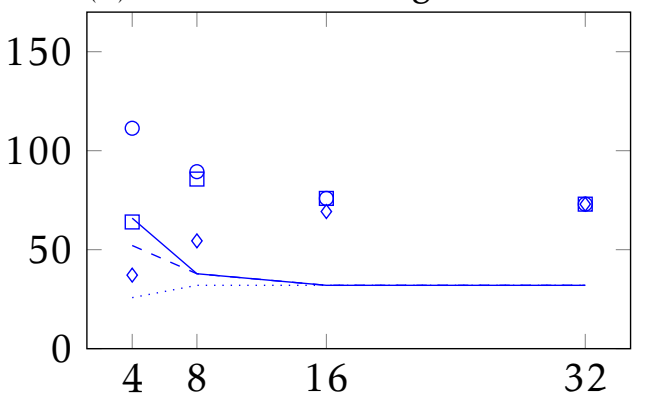

(b) HC-128 using FIFO.

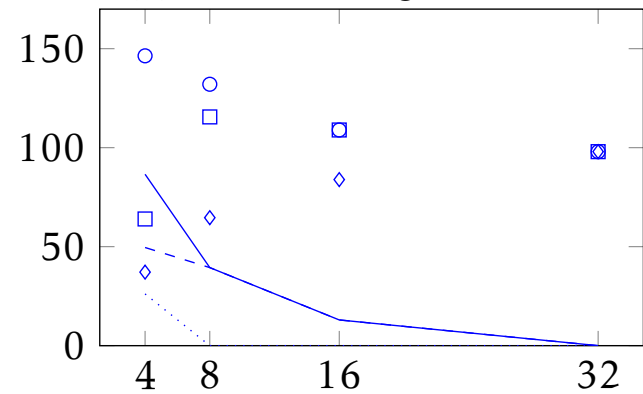

(c) Sosemanuk using LRU.

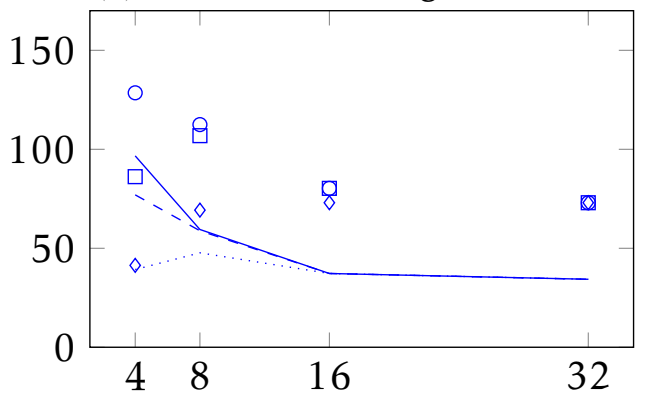

(d) HC-128 using LRU.

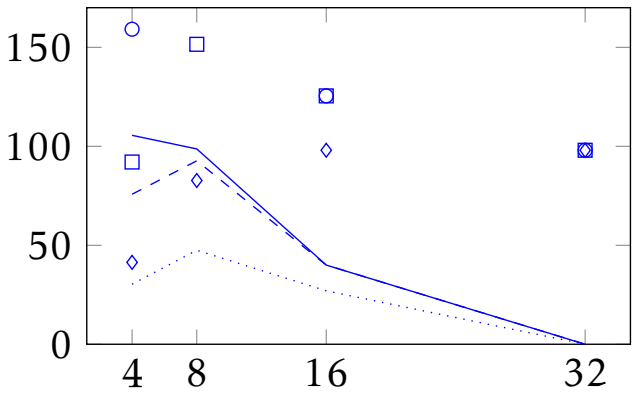

(e) Sosemanuk PLRU.

(f) HC-128 using PLRU.

\begin{tabular}{|lcc|}
\hline$\circ \quad$ Upper bound on Absorption & Computed Absorption \\
$\square$ Upper bound on Extraction (Shared) & -- Computed Extraction (Shared) \\
$\diamond$ Upper bound Extraction (Disjoint) & $\cdots . . . .$. & Computed Extraction (Disjoint)
\end{tabular}

Figure 6.2: Information absorption and extraction (in bits) for the Sosemanuk and HC-128 execution on a 4-way cache with line size of 64 bytes. Figures $6.2 \mathrm{a}$, $6.2 \mathrm{c}$ and $6.2 \mathrm{e}$ depict the leakage of Sosemanuk, 6.2b, 6.2d and 6.2f depict the leakage of $\mathrm{HC}-128$. The horizontal axis depicts the size of the cache in $\mathrm{KB}$, the vertical axis depicts the extracted information in logarithmic scale, that is, converting the number observations into bits of information. Points represent the upper bounds on the leakage, assuming an even distribution of the memory blocks and considering the worst-case scenario. Lines represent the values computed by CacheAudit. 


\subsection{Upper Bounds for Sosemanuk and HC-128}

In this Section we analyze the security of HC-128 and Sosemanuk using CacheAudit, compare one to the other for different configurations of the cache and compare them to the upper bounds when using worst-case analysis, where we see a large difference in leakage in contrast to what we saw for AES.

Figure 6.2 depicts the upper bounds of the leakage alongside with the values computed by CacheAudit when analyzing Sosemanuk and HC-128 starting on an empty cache. Here we highlight the following results.

- We improve the bounds on the information leakage when computing the information extraction on the set of reachable configurations computed by CacheAudit. The difference between the information absorption and the information extraction corresponds to the precision gained by the development of the extraction Algorithm 1. This gain is generally higher when considering small caches, where sets contain several memory blocks, and with LRU and PLRU, see Figures 6.2c, 6.2d, 6.2e and $6.2 f$.

- We see that, depending on the size of the cache, one cryptographic algorithm is better than the other:

- For small caches (cache size of $4 \mathrm{~KB}$ ), both algorithms have similar values for the extraction, both shared and disjoint, although HC-128 has a larger absorption.

The difference in information absorption is due to $\mathrm{HC}-128$ having a larger footprint than that of Sosemanuk.

The similarities in information extraction are due to the theoretical upper bounds presented in Section 5.2.2. Both cryptographic algorithms use the same cache, thus the same associativity and number of cache sets. Since the information absorption per set is large enough for both cryptographic algorithms, most of the cache sets reach the bounds in information extraction for both cryptographic algorithms.

- As the size of the cache grows, the absorption and extraction for HC128 gets smaller than that of Sosemanuk (to the point of no leakage when the cache size is $32 \mathrm{~KB}$ ). This was already shown in [DKMR15] and is due to the fact that HC-128, unlike AES and Sosemanuk, does not store a precomputed lookup table in the cache but one that it modifies throughout the computation. Then, by continually generating the S-boxes, HC-128 ensures that every memory block is freshly loaded in the cache. As the cache grows enough so that accesses to the lookup table do not conflict with each other, the absorption goes to zero. 
- When comparing the computed values of CacheAudit with the upper bounds we see very large differences. This is because HC-128 and Sosemanuk reach very few configurations of the possible ones computed by the worst-case scenario analysis. Moreover, the way that HC-128 modifies its lookup tables makes its leakage for large caches closer to the worst-case leakage of a filled cache than that of an empty cache. Opposite to the case of AES, the use of CacheAudit for HC-128 and Sosemanuk gives significantly better security guaranties for these cryptographic algorithms than if we approximate them with the upper bounds obtained by worst-case scenario analysis. 


\section{CHAPTER 7}

\section{COMPETITIVE ANALYSIS}

Worst-case analysis provides results on a program that are independent of the input of the program but has one main downside: programs are analyzed based on one single trace of memory blocks (or set of traces in the case of security) that may not represent the entire spectrum of executions accurately.

Consider for example two cache algorithms, $\mathrm{P}$ and $\mathrm{Q}$ such that $\mathrm{P}$ leaks consistently more information than $Q$ for all sets of traces of memory blocks except for one set of traces, where both algorithms leak a similar amount of information. When using worst-case analysis, we would conclude that both algorithms are similar in leakage when, most of the time, $Q$ is better than $P$.

Our objective in this chapter is to define a notion for comparing pairs of cache algorithms that emphasizes the difference between two cache algorithms. For that, we take inspiration from the classic notion of competitiveness [ST85], which compares an online algorithm with the optimal offline algorithm and gives a bound on the largest difference between them, and the notion of relative miss competitiveness [RG08] from the real-time systems community, that adapts classic competitiveness to two online algorithms. We define a measure for comparing the security of cache algorithms, which we call the leak ratio, that bounds the largest difference in leakage between two cache algorithms.

When considering the time based attacker and cache algorithms represented by the automata model, we show that the leak ratio is symmetric in the cache algorithms, that is, no cache algorithms dominates another in terms of timing leakage. This is surprising considering that, for performance, such differences exist [ST85]. Moreover, when restricting to cache algorithms with finite control, which is natural for hardware based cache implementation, the leak ratio is either constant or asymptotically linear in the length of the traces, no other shapes are possible.

When considering the access-based attacker, we use some of the upper bounds obtained in Chapter 5 to show that, for some cache algorithms, the 
leak competitiveness relation is symmetric as in the case of time-based attacker and, for some others, there is a dominance of one cache algorithm over the other.

These conclusions are similar to the ones obtained for the upper bounds in Chapter 5 where we saw that all cache algorithms under the time based attacker have the same upper bound, Corollary 5.2, but these upper bounds differ between cache algorithms under the access-based attacker.

We now introduce the notion of relative miss competitiveness used for performance evaluation and then adapt it to the different attackers.

\section{Relative Miss Competitiveness}

Relative competitiveness is a notion for comparing the worst-case performance of two cache algorithms. Below, we reproduce a slightly simplified version of the definition of relative competitiveness from [RG08]:

Definition 7.1. For $\rho \in \mathbb{R}_{>0}$, we say that a algorithm $\mathrm{P}$ is $\rho$-miss-competitive relative to algorithm $Q$ if there exists $c \in \mathbb{R}_{>0}$ such that

$$
\mathrm{P}(t) \leq \rho \cdot \mathrm{Q}_{\mathrm{tme}}(t)+c,
$$

for all traces $t \in B^{*}$.

Example 7.2. LRU of associativity 4 is 1-miss-competitive relative to FIFO of associativity 2. On the other hand, FIFO of associativity 2 is not $\rho$-misscompetitive to LRU of associativity 4 for any $\rho$. Therefore, LRU of associativity 4 outperforms FIFO of associativity 2 in number of misses. See [RG08] for details and more examples.

Notions of performance such as relative competitiveness (see Definition 7.1) are based on trace properties, hence the point of comparison are individual traces. In contrast, information-theoretic notions of leakage are hyperproperties, which makes sets of traces the natural point of comparison. Given an attacker, we define leak competitiveness, a concept that enables us to compare the leakage of two cache algorithms, and that is based on lifting miss competitiveness from traces to sets of traces.

\subsection{Time-based Attacker}

Time-based attackers monitor the effect of the trace of memory blocks in the cache, the attacker observes the total number of misses the trace of memory blocks produces, without knowing which particular memory blocks produced the miss. 
In this section we define leak competitiveness for an automata-based cache algorithm under the time-based attacker model and study the competitiveness relationships between pairs of cache algorithms.

Since the sets of observations obtained for the time and trace-based attackers grow with the length of the traces of memory blocks, we abstract from programs to a set of traces of equal length $T \subseteq B^{l}$. The information the program leaks through timing is captured by $P_{\text {tme }}(T) \subseteq \mathbb{N}$, the image of $T$ under $\mathrm{P}$, and quantified by $\left|\mathrm{P}_{\text {tme }}(T)\right| \in \mathbb{N}$.

\subsubsection{Leak Ratio}

We next introduce a notion based on relative competitiveness that compares the amount of information that two cache algorithms leak via their timing behavior.

Definition 7.3. For a function $\rho^{\text {tme }}: \mathbb{N} \rightarrow \mathbb{R}_{\geq 0}$, we say that algorithm $P$ is $\rho^{\text {tme }}$ leak-competitive relative to algorithm $Q$ for a time-based attacker if, for all $l \in \mathbb{N}$,

$$
\left|\mathrm{P}_{\mathrm{tme}}(T)\right| \leq \rho^{\mathrm{tme}}(l) \cdot\left|\mathrm{Q}_{\mathrm{tme}}(T)\right|
$$

for all set of traces of blocks $T \subseteq B^{l}$. Here we use $\mathrm{P}_{\text {tme }}(T)$ as a shortcut for $P_{\text {tme }}\left(\left(i_{P}, \lambda j . \perp\right), T\right)$, i.e., the number of misses on the set of traces $T$ when starting in the initial configuration of the cache.

Even though the definition of leak competitiveness is based on a lifting of miss competitiveness, there are important differences. Most importantly, leak competitiveness of two algorithms P, $Q$ bounds the ratio of leakage for each $l \in \mathbb{N}$, whereas miss competitiveness bounds the ratio of hits and misses for all $l$. For traces of length $l$ and an empty initial cache, the number of misses any cache algorithm can produce is in $\{1, \ldots, l\}$, which means that any two algorithms are $\rho^{\text {tme }}$-leak-competitive for $\rho^{\text {tme }}(l)=l$. The question is hence not whether there two algorithms are leak-competitive, but rather what shape this relationship takes. We introduce the leak ratio to facilitate reasoning about this shape.

Definition 7.4. Given a pair of algorithms $\mathrm{P}$ and $\mathrm{Q}$ we define the leak ratio for a time-based attacker $\rho_{\mathrm{P}, 0}^{\mathrm{tme}}$ as:

$\rho_{\mathrm{P}, \mathrm{Q}}^{\mathrm{tme}}(l)=\min \left\{\rho^{\mathrm{tme}}(l) \mid \rho^{\mathrm{tme}}: \mathbb{N} \rightarrow \mathbb{R}_{\geq 0}, \mathrm{P}\right.$ is $\rho^{\mathrm{tme}}$-leak-competitive relative to $\left.Q\right\}$.

As we are mostly interested in the asymptotic behavior of $\rho_{\mathrm{P}, 0}^{\mathrm{tme}}$, the lack of an additive slack in the definition of miss competitiveness is not essential. 


\subsubsection{Characterizing the Leak Ratio}

In this section we present our main result, which is a characterization of the asymptotic behavior of the leak ratio for any pair of cache algorithms. We then give interpretations of this behavior in terms of security. We present the proofs of the technical results in Sections 7.1.4-7.1.6.

\section{Non-Dominance}

The key question motivating our work is whether some cache algorithms are preferable to others in terms of their leakage via timing. This is a natural question to ask because it is well-known that such preferences relations exist for performance, see Example 7.2. The following theorem gives a negative answer to the question above.

Theorem 7.5. For each pair of algorithms $\mathrm{P}, \mathrm{Q}$ we have, as 1 grows:

$$
\mathcal{O}\left(\rho_{\mathrm{P}, 0}^{\mathrm{tme}}(l)\right)=\mathcal{O}\left(\rho_{0, \mathrm{P}}^{\mathrm{tme}}(l)\right) .
$$

Theorem 7.5 shows that cache algorithms are incomparable in the sense that, for every $l \in \mathbb{N}$ and every set of traces $T$ that witnesses an advantage for $P$ over $Q$ in terms of leakage, there is a set of traces $T^{\prime}$ that witnesses a comparable advantage of $Q$ over $P$. The following examples exhibits such witnesses for $\mathrm{P}=\mathrm{LRU}$ and $\mathrm{Q}=\mathrm{FIFO}$.

Example 7.6. Consider two fully-associative caches of capacity two, one with LRU and the other with FIFO replacement, and the following sets of traces:

$$
T=\left\{\begin{array}{l}
\text { ABACACBBB, } \\
\text { ABACDAAAA, } \\
\text { ABACBADDD, } \\
\text { ABACBACBB, } \\
\text { ABACBACBA }
\end{array}\right\} \quad T^{\prime}=\left\{\begin{array}{l}
\text { ABACBAAAA }, \\
\text { ABACDAAAA, } \\
\text { ABACABCCC, } \\
\text { ABACACBCA }
\end{array}\right\}
$$

Starting from an empty initial cache state, LRU produces 5 different observations on $T$, whereas FIFO produces only one. In contrast, FIFO produces 4 different observations on $T^{\prime}$ whereas LRU produces only one.

The root cause for this divergent behavior is that, after accessing the prefix $\mathrm{ABAC}$, the content of both caches differs: block $\mathrm{C}$ evicts the least recently used block for LRU (i.e., B) but the first block to enter the cache for FIFO (i.e., A). The suffixes of the traces are constructed in such a way that the difference in cache content maps to different observable behavior. The full diagram of updates of the cache when using these sets of traces is given in Figure A.1 in the Appendix. 
The proof of Theorem 7.5 is based on a systematic way of constructing sets of traces such as the ones in Example 7.6. Formally, the Theorem follows from applying Theorem 7.15, introduced in Section 7.1.5, to P, Q and to Q,P. Moreover, we will see later that these sets can be obtained from only two traces of memory blocks and that those two traces are enough to characterize the leak ratio.

\section{Shapes of $\rho_{P, Q}^{\text {tme }}$}

In Section 7.1.1 we have already observed that the leak ratio $\rho_{\mathrm{P}, 0}^{\text {tme }}$ between any two algorithms $P$ and $Q$ is upper bounded by a linear function. The interesting question is hence what sublinear shapes $\rho_{\mathrm{P}, 0}^{\mathrm{tme}}$ can take. We answer this question for cache algorithms with finite sets of control states, which encompasses most hardware-based cache implementations. For this important class, the following theorem shows that the leak ratio is either asymptotically constant or linear, ruling out any nontrivial sublinear shape.

Theorem 7.7. For each pair of algorithms $\mathrm{P}, \mathrm{Q}$ with finite control we have either

- $\rho_{\mathrm{P}, \mathrm{Q}}^{\mathrm{tme}}(l) \in \Theta(l)$, or

- $\rho_{\mathrm{P}, 0}^{\mathrm{tme}}(l) \in \Theta(1)$.

These results are a direct consequence of Theorem 7.19, introduced in Section 7.1.6, which shows that the leak ratio of two finite-control algorithms $P, Q$ is lower bounded by a linear factor if and only if there exist traces that witness that the difference in misses between $P$ and $Q$ is unbounded. Whether such traces exist determines in which of the two classes described by Theorem 7.7 the algorithms $P$ and $Q$ fall. If they do not exist, note that Corollary 7.14 implies that $\rho_{\mathrm{P}, 0}^{\mathrm{tme}} \in \mathcal{O}(1)$.

For example, any pair of algorithms with different capacities falls into the first class. This is because one algorithm always contains a block that the other does not, which allows to construct a trace of unbounded difference in misses.

Together with Theorem 7.5, Theorem 7.7 leads to a stronger non-dominance result for finite-control algorithms, namely that for every $l \in \mathbb{N}$ there are sets of traces $T_{\mathrm{P}}^{l}, T_{0}^{l} \in B^{l}$ such that one algorithm asymptotically leaks the largest possible amount of information whereas the other leaks almost nothing. That is, $\mathrm{P}_{\mathrm{tme}}\left(T_{\mathrm{P}}^{l}\right) \in \Theta(1)$ and $\mathrm{Q}_{\mathrm{tme}}\left(T_{\mathrm{P}}^{l}\right) \in \Theta(l)$, whereas $\mathrm{P}_{\mathrm{tme}}\left(T_{0}^{l}\right) \in \Theta(l)$ and $\mathrm{Q}_{\mathrm{tme}}\left(T_{0}^{l}\right) \in \Theta(1)$.

The non-dominance results from Theorems 7.5 and 7.7 also show why we cannot define leak competitiveness in the same way as miss-competitiveness, that is, where each pair of cache algorithms has a constant leak ratio for all lengths of traces. Except for the case where both leak ratios are in $\Theta(1)$, if we can find, for each length, sets of traces where one cache algorithm leaks 


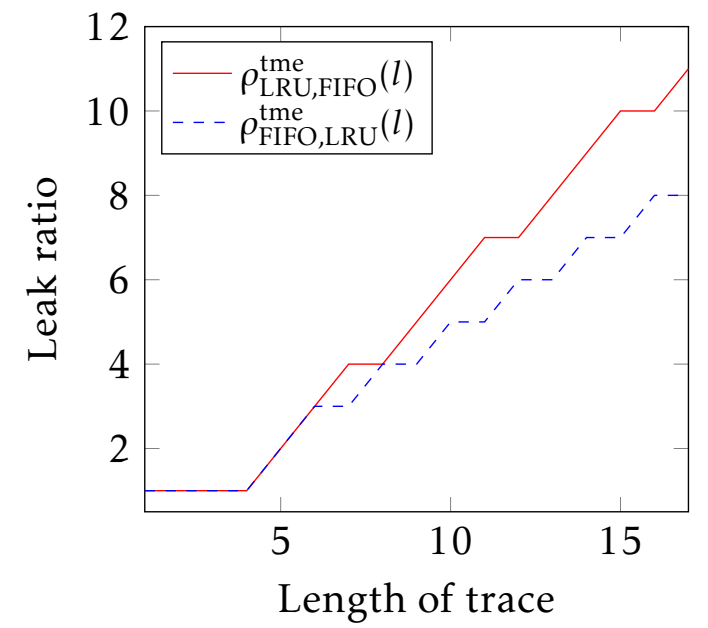

(a) Comparison of the leak ratios of LRU relative to FIFO and vice versa.

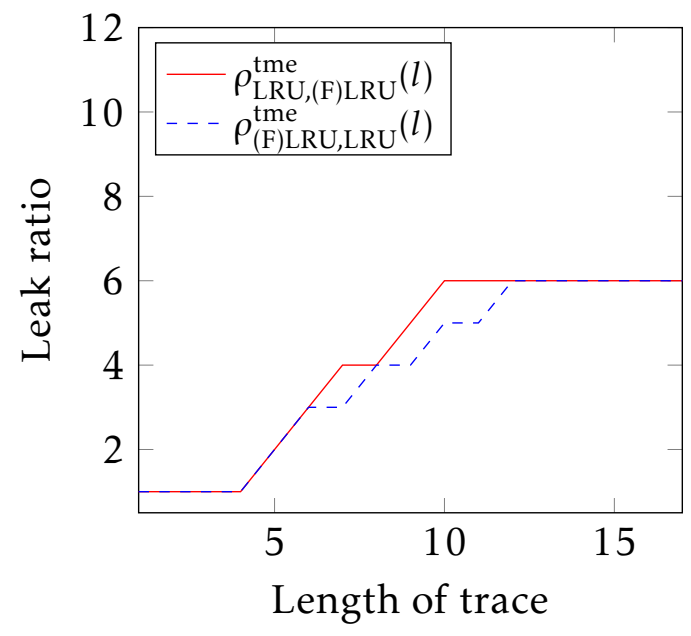

(b) Comparison of the leak ratios of LRU relative to (F)LRU and vice versa.

Figure 7.1: Example of the behavior of the leak ratios of the cache algorithms from Example 7.8. Figure 7.1a shows two leak ratios that grow asymptotically linearly and where the slopes can give a slight advantage of one algorithm over the other. Figure $7.1 \mathrm{~b}$ shows two leak ratios that eventually become constant functions and were, for large lengths of traces, there is no advantage of one algorithm over the other.

more and more information as we increase the length whereas the other leaks a constant amount, no constant value of the leak ratio satisfies the leak-competitiveness definition for all lengths.

We now compute the leak ratio functions for two pairs of cache algorithms to showcase how the constants ignored in the asymptotic results in Theorems 7.5 and 7.7 can mean a small advantage of one cache algorithm over the other, provided the leak ratios are not in $\Theta(1)$.

Example 7.8. We can compute the leak ratios for small lengths of traces by computing all traces of hits and misses of a given length and then choosing the subset of traces that produces the largest ratio in the number of observations in favor of each algorithm.

To compute these traces of hits and misses we simulate them by exhaustively enumerating all possible traces of memory blocks $B^{l}$. We now argue the size of the set $B$ needed for the case of capacity two. For every pair of configurations updated from the initial by the same trace of memory blocks, the last accessed block is cached (2.1), accessing this block again produces a hit for both configurations. The other line of the configuration may store a different memory block for each cache algorithm so that accessing one of them produces a hit for one algorithm and a miss for the other and vice versa. Fi- 
nally, accessing any memory block not cached for any of the cache algorithms produces a miss for both. Then, for cache algorithms with capacity two, four memory blocks are enough to simulate all possible traces of hits and misses.

Consider two pairs of fully-associative caches of capacity two, one pair considers replacements LRU and FIFO while the other considers LRU and a cache algorithm that we denote (F)LRU that starts behaving like FIFO but, after seven accesses to memory, behaves like LRU for the remaining of the accesses. The leak ratios for LRU and FIFO are shown in Figure 7.1a and the ones for LRU and (F)LRU are shown in Figure 7.1b.

We see that the leak ratios of LRU and FIFO exemplify the first case of Theorem 7.7 and that, by looking at the slopes, that the asymptotic approach ignores, we conclude that FIFO has a small advantage over LRU since the leak ratio of FIFO relative to LRU grows slower than that of LRU relative to FIFO, Figure 7.1a.

On the other hand, the leak ratios of LRU and (F)LRU exemplify the second case of Theorem 7.7. The leak ratios for both cache algorithms start growing at different rates but, once both algorithms behave like LRU, the leak ratios end up coinciding and become constant functions. Although both cache algorithms behave the same starting from length eight, not all pairs of configurations contain the same memory blocks at this point, which still allows for both leak ratios to grow with the length. Once all traces update the pairs of configurations to having the same memory blocks for both algorithms, the leak ratios become constant functions.

A pair of cache algorithms that are the same or eventually become the same are the only ones that verify the second case of Theorem 7.7. This is because the leak ratios grow when the pairs of configurations do not have the same memory blocks cached and the access to a specific memory block produces a hit for one algorithm and a miss for the other. If, at some point, every access to a memory block has the same effect for both algorithms, the leak ratios do not grow anymore.

By using a constant notion of leak competitiveness, all cache algorithms, except for the ones in $\Theta(1)$, would be deemed incomparable. On the other hand, by defining the leak ratios as functions of the length of the trace and observing the growth rate as in Example 7.8, we can establish a comparison between cache algorithms.

\subsubsection{Discussion}

We now discuss the implications of Theorems 7.5 and 7.7 in practice.

1. Our results are asymptotic in nature. The constants hidden behind the $\mathcal{O}$-notation can indicate a (gradual) preference between algorithms on 
finite sets of traces. E.g., the traces in Example 7.6 and the different slopes on Example 7.8 show a slight advantage of FIFO over LRU.

2. Our results rely on the construction of sets of traces that witness advantages of one cache algorithm over another, see Example 7.6. However, the constructed traces need not correspond to a program of interest. Restricting to a specific class of programs corresponds to the constraint that witnesses be picked from a subset $T \subseteq B^{l}$ instead of $B^{l}$. Under such constraints, it may be possible that a preference relation between cache algorithms exists.

3. Our results rely on the assumptions that the caching algorithm is deterministic and based on demand paging, i.e., it loads blocks only when they are requested by the program. It is possible that randomized policies or features such as prefetching enable one to sidestep our results. For example, for miss-competitiveness it is known that randomized policies $\left[\mathrm{FKL}^{+} 91\right]$ achieve better bounds than those possible for deterministic policies [ST85]. The study of leak competitiveness for randomized cache algorithms is out of the scope of our work. We are aware of nondominance results for a similar notion of leak-competitiveness that consider the RANDOM cache algorithm that, upon a miss, evicts a memory block randomly, see [Sch18].

\subsubsection{Leak Ratio from a Pair of Traces}

Information leakage is a hyperproperty, i.e., a property of sets of traces. We now show that the leak can always be expressed in terms of the difference in observations of only two traces of memory blocks.

For an algorithm $Q$ and $l \in \mathbb{N}$, we say that $t_{1}, t_{2} \in B^{l}$ are Q-equivalent whenever $Q_{\text {tme }}\left(t_{1}\right)=Q_{\text {tme }}\left(t_{2}\right)$. We say that a set $T \subseteq B^{l}$ is $Q$-dense if the image of $T$ under $Q$ is a contiguous sequence of natural numbers, i.e. $Q_{t m e}(T)=\{j, j+$ $1, \ldots, j+k\}$ for some $j, k \in \mathbb{N}$.

Proposition 7.9. For all pairs of algorithms $\mathrm{P}$ and $\mathrm{Q}$, all lengths $\mathrm{l}$, and all pairs of Q-equivalent traces of memory blocks $t_{1}, t_{2} \in B^{l}$ :

$$
\mathrm{P}_{\mathrm{tme}}\left(t_{2}\right)-\mathrm{P}_{\mathrm{tme}}\left(t_{1}\right) \leq \rho_{\mathrm{P}, \mathrm{t}}^{\mathrm{tme}}(l)-1 \text {. }
$$

Moreover, there exist pairs of traces of Q-equivalent memory blocks $t_{1}, t_{2} \in B^{l}$ such that (7.1) is an equality.

That is, every pair of traces that coincides in timing observation on one algorithm cannot differ by more than the leak ratio on the other algorithm. Moreover, there exists a pair of traces that matches this bound. 
The proof of the upper bound is based on constructing a set $T \subseteq B^{l}$ of Qequivalent traces from a pair $t, t^{\prime} \in B^{l}$ of $Q$-equivalent traces. The set $T$ is $\mathrm{P}$ dense with maximum $\mathrm{P}\left(t^{\prime}\right)$ and minimum $\mathrm{P}_{\text {tme }}(t)$. It satisfies

$$
\rho_{\mathrm{P}, \mathrm{Q}}^{\mathrm{tme}}(l) \geq \frac{\left|\mathrm{P}_{\mathrm{tme}}(T)\right|}{\left|Q_{\mathrm{tme}}(T)\right|},
$$

which equals $\mathrm{P}_{\text {tme }}(t)-\mathrm{P}_{\text {tme }}\left(t^{\prime}\right)+1$ by construction.

The following lemma describes the construction of traces Q-equivalent to $t$ and $t^{\prime}$ whose number of misses for $\mathrm{P}$ cover every value in between $\mathrm{P}_{\text {tme }}(t)$ and $\mathrm{P}_{\text {tme }}\left(t^{\prime}\right)$. The set $T$ is composed of these traces.

Lemma 7.10. Consider two Q-equivalent traces $t, t^{\prime} \in B^{l}$ with $\mathrm{P}_{\text {tme }}(t) \leq \mathrm{P}_{\text {tme }}\left(t^{\prime}\right)$. Then, for every $\mathrm{P}_{\mathrm{tme}}(t) \leq k \leq \mathrm{P}_{\mathrm{tme}}\left(t^{\prime}\right)$ there exists a trace $t^{*} \in B^{l}$ such that $\mathrm{P}_{\mathrm{tme}}\left(t^{*}\right)=$ $k$ and that is Q-equivalent to $t$ and $t^{\prime}$.

Proof. We begin with a continuity argument to identify a prefix of the trace $t$, which we later extend to $t^{*}$. For this, note that the difference in misses, $Q-P$, between both algorithms on trace $t$ is initially zero, i.e. $Q_{\text {tme }}(\epsilon)-P_{\text {tme }}(\epsilon)=$ 0 , and increases or decreases by at most 1 per added block, until it reaches $Q_{\text {tme }}(t)-P_{\text {tme }}(t)$. We first consider the case $Q_{\text {tme }}(t) \geq P_{\text {tme }}(t)$. For any $k$ with $0 \leq Q_{\text {tme }}(t)-k \leq Q_{\text {tme }}(t)-P_{\text {tme }}(t)$, we hence find a prefix $b_{1} \cdots b_{u}$ of $t$ such that the value of $Q-P$ on the prefix is exactly $Q_{t m e}(t)-k$ :

$$
Q_{\mathrm{tme}}\left(b_{1} \cdots b_{u}\right)-\mathrm{P}_{\mathrm{tme}}\left(b_{1} \cdots b_{u}\right)=Q_{\mathrm{tme}}(t)-k \text {. }
$$

We create a trace $t^{*}$ with prefix $b_{1}^{*} \ldots b_{u}^{*}=b_{1} \ldots b_{u}$, which we extend by blocks $b_{u+1}^{*} \ldots b_{v}^{*}$ that produce misses on both $\mathrm{P}$ and $\mathrm{Q}$ until

$$
Q_{\text {tme }}\left(b_{1}^{*} \ldots b_{v}^{*}\right)=Q_{\text {tme }}(t) .
$$

For the blocks $b_{u+1}^{*} \ldots b_{v}^{*}$ to miss they must be uncached in both $\mathrm{P}$ and 0 ; such blocks can be found whenever $B$ is larger than the sum of the the capacities of both algorithms. We further extend $b_{1}^{*} \ldots b_{v}^{*}$ with $l-v$ copies of $b_{v}^{*}$ to the trace $t^{*}$ of length $l$. Repeatedly accessing $b_{v}^{*}$ is guaranteed to produce hits on both $\mathrm{P}$ and Q.

As the blocks $b_{u+1}^{*} \ldots b_{l}^{*}$ produce identical outputs on $\mathrm{P}$ and $\mathrm{Q}$, the trace $t^{*}$ still satisfies (7.3), i.e.,

$$
Q_{\text {tme }}\left(t^{*}\right)-P_{\text {tme }}\left(t^{*}\right)=Q_{\text {tme }}(t)-k .
$$

Moreover, $t^{*}$ also still satisfies (7.4), i.e., $Q_{\text {tme }}\left(t^{*}\right)=Q_{\text {tme }}(t)$, from which we conclude that $t^{*}$ is Q-equivalent to $t$ and $\mathrm{P}_{\text {tme }}\left(t^{*}\right)=k$. Note that we only handled the case $k \leq Q_{\text {tme }}(t)$. The case $k>Q_{\text {tme }}(t)$ proceeds in the same way but extending a prefix of $t^{\prime}$ instead of $t$ and reformulating (7.3) to $P_{\text {tme }}\left(b_{1}^{\prime} \cdots b_{u}^{\prime}\right)-Q_{\text {tme }}\left(b_{1}^{\prime} \cdots b_{u}^{\prime}\right)=$ $k-Q_{\text {tme }}\left(t^{\prime}\right)$. 
Example 7.11. Note that the sets of traces introduced in Example 7.6 are obtained following the construction presented in the previous proof. For example the first set:

\section{\{ABACACBBB, ABACDAAAA, ABACBADDD,} ABACBACBB, ABACBACBA\}.

Given the last trace, ABACBACBA, one can construct all the other traces except for the first one by taking a prefix of ABACBACBA and then completing the trace with memory blocks that produce the same output for both algorithms, see Figure A.1 for the effect every memory block produces on the cache.

The proof of the tightness of the upper bound in Proposition 7.9 follows from the fact that every set $T$ that satisfies equality in (7.2) contains within it a subset $T^{*}$ of Q-equivalent traces that also satisfies equality in (7.2). We show that this set $T^{*}$ is P-dense, which means that the elements $t, t^{\prime} \in T^{*}$ that produce the maximal difference in misses under $\mathrm{P}$ satisfy $\mathrm{P}_{\mathrm{tme}}(t)-\mathrm{P}_{\mathrm{tme}}\left(t^{\prime}\right)=\rho_{\mathrm{P}, 0}^{\mathrm{tme}}-1$.

The following lemma shows how to find such a $T^{*}$.

Lemma 7.12. Every set $T \subseteq B^{l}$ that satisfies equality in (7.2) contains a $\mathrm{P}$-dense subset of Q-equivalent traces that also satisfies equality in (7.2).

Proof. We partition $T=T_{1} \uplus \cdots \uplus T_{k}$, into classes of Q-equivalent traces. Without loss of generality assume that $P_{\text {tme }}\left(T_{1}\right) \geq P_{\text {tme }}\left(T_{j}\right)$, for $j>1$. Then we have:

$$
\frac{\left|\mathrm{P}_{\mathrm{tme}}(T)\right|}{\left|\mathrm{Q}_{\mathrm{tme}}(T)\right|} \leq \frac{\sum_{j=1}^{k}\left|\mathrm{P}_{\mathrm{tme}}\left(T_{j}\right)\right|}{\sum_{j=1}^{k}\left|\mathrm{Q}_{\mathrm{tme}}\left(T_{j}\right)\right|}=\frac{\sum_{j=1}^{k}\left|\mathrm{P}_{\mathrm{tme}}\left(T_{j}\right)\right|}{\sum_{j=1}^{k} 1} \stackrel{(*)}{\leq}\left|\mathrm{P}_{\mathrm{tme}}\left(T_{1}\right)\right|,
$$

where $(*)$ follows from the fact that, for any sequence of natural numbers $a_{1}, \ldots, a_{k}, \sum_{j=1}^{k} a_{j} \leq k \max \left(a_{1}, \ldots, a_{k}\right)$.

As a consequence, $T_{1}$ also satisfies $\left|P_{\text {tme }}\left(T_{1}\right)\right|=\rho_{P, Q}^{\text {tme }}(l)$. Moreover, $P_{\text {tme }}\left(T_{1}\right)$ is a contiguous set of natural numbers. If it were not, we could apply Lemma 7.10 to augment $T_{1}$ by a trace that produces the missing number of observations, contradicting that $\rho_{\mathrm{P}, 0}^{\mathrm{tme}}$ is an upper bound.

\subsubsection{Approximation of the Leak Ratio from a Single Trace}

In Section 7.1.4 we have seen that the leak ratio of two cache algorithms, which is defined as a property of arbitrary sets of traces, is fully characterized by a pair of traces. In this section, we show that the leak ratio can be approximated to within a factor of 2 using a single trace.

Lemma 7.13. Let $t \in B^{l}$ be an arbitrary trace. Then, there is a trace $t^{\prime} \in B^{l}$ with $P_{\text {tme }}\left(t^{\prime}\right)=Q_{\text {tme }}\left(t^{\prime}\right)=Q_{\text {tme }}(t)$. 
Proof. We construct the trace $t^{\prime} \in B^{l}$ as the concatenation of two subtraces $t_{\text {miss }}^{\prime}$ and $t_{\text {hit }}^{\prime}: t_{\text {miss }}^{\prime}$ is a trace of length $Q_{\text {tme }}(t)$ in which all accesses are chosen such that they result in misses in both $\mathrm{P}$ and $\mathrm{Q}$. This is always possible, as there are at most $\eta_{P}+\eta_{Q}$ blocks cached in $P$ and $Q$ at any time and accesses to any other block will result in a miss. Let $b \in B$ be the final access in $t_{\text {miss }}^{\prime}$. Independently of the cache algorithm, $b$ must be cached in both $\mathrm{P}$ and $\mathrm{Q}$ following $t_{\text {miss }}^{\prime}$. The second subtrace $t_{\text {hit }}^{\prime}$ then simply consists of $|t|-Q_{\text {tme }}(t)$ accesses to $b$, which will result in hits in both $P$ and 0 .

The following Corollary of Lemma 7.13 and of Proposition 7.9 shows that the leakage ratio is "almost" a trace property, as it can be approximated to within a factor of two based on the number of misses of $P$ and $Q$ on a single trace:

Corollary 7.14. For all pairs of cache algorithms $\mathrm{P}$ and $\mathrm{Q}$, all lengths $\mathrm{l}$, and all traces of memory blocks $t \in B^{l}$ :

$$
\left|\mathrm{P}_{\mathrm{tme}}(t)-Q_{\mathrm{tme}}(t)\right| \leq \rho_{\mathrm{P}, 0}^{\mathrm{tme}}(l)-1 .
$$

Moreover, there exists a trace $t \in B^{l}$ such that:

$$
\frac{\rho_{\mathrm{P}, 0}^{\mathrm{tme}}(l)-1}{2} \leq\left|\mathrm{P}_{\mathrm{tme}}(t)-Q_{\mathrm{tme}}(t)\right| \text {. }
$$

Proof. Let $t \in B^{l}$ be an arbitrary trace. By Lemma 7.13, there is a trace $t^{\prime}$ such that $\mathrm{P}_{\text {tme }}\left(t^{\prime}\right)=Q_{\text {tme }}\left(t^{\prime}\right)=Q_{\text {tme }}(t)$. So $t$ and $t^{\prime}$ are Q-equivalent. Thus, by Proposition 7.9 , we have both

$$
\mathrm{P}_{\text {tme }}(t)-\mathrm{P}_{\text {tme }}\left(t^{\prime}\right) \leq \rho_{\mathrm{P}, 0}^{\mathrm{tme}}(l)-1 \quad \text { and } \quad \mathrm{P}_{\text {tme }}\left(t^{\prime}\right)-\mathrm{P}_{\text {tme }}(t) \leq \rho_{\mathrm{P}, 0}^{\mathrm{tme}}(l)-1,
$$

which implies that $\left|P_{\text {tme }}(t)-Q_{\text {tme }}(t)\right|=\left|P_{\text {tme }}(t)-P_{\text {tme }}\left(t^{\prime}\right)\right| \leq \rho_{\mathrm{P}, 0}^{\text {tme }}(l)-1$.

By Proposition 7.9, there is a pair of $Q$-equivalent traces $t_{1}, t_{2} \in B^{l}$ such that:

$$
\mathrm{P}_{\mathrm{tme}}\left(t_{2}\right)-\mathrm{P}_{\mathrm{tme}}\left(t_{1}\right)=\rho_{\mathrm{P}, 0}^{\mathrm{tme}}(l)-1 \text {. }
$$

Let $q=Q_{\text {tme }}\left(t_{1}\right)=Q_{\text {tme }}\left(t_{2}\right)$. Either $2 \cdot\left|P_{\text {tme }}\left(t_{2}\right)-q\right| \geq \mathrm{P}_{\text {tme }}\left(t_{2}\right)-\mathrm{P}_{\text {tme }}\left(t_{1}\right)$ or 2 . $\left|P_{\text {tme }}\left(t_{1}\right)-q\right| \geq P_{\text {tme }}\left(t_{2}\right)-P_{\text {tme }}\left(t_{1}\right)$, where equality is achieved on one of the two inequalities if $q$ is centered between $P_{\text {tme }}\left(t_{1}\right)$ and $P_{\text {tme }}\left(t_{2}\right)$. Assume that 2 . $\left|P_{\text {tme }}\left(t_{2}\right)-q\right| \geq P_{\text {tme }}\left(t_{2}\right)-P_{\text {tme }}\left(t_{1}\right)$. Then $\left|P_{\text {tme }}\left(t_{2}\right)-Q_{\text {tme }}\left(t_{2}\right)\right| \geq \frac{P_{\text {tme }}\left(t_{2}\right)-P_{\text {tme }}\left(t_{1}\right)}{2}=$ $\frac{\rho_{\mathrm{P}, 0}^{\mathrm{tme}}-1}{2}$. Otherwise, $\left|\mathrm{P}_{\mathrm{tme}}\left(t_{1}\right)-Q_{\mathrm{tme}}\left(t_{1}\right)\right| \geq \frac{\mathrm{P}_{\mathrm{tme}}\left(t_{2}\right)-\mathrm{P}_{\mathrm{tme}}\left(t_{1}\right)}{2}=\frac{\rho_{\mathrm{P}, 0}^{\mathrm{tme}}-1}{2}$.

Theorem 7.15. For all pairs of cache algorithms $\mathrm{P}$ and $\mathrm{Q}$ and all lengths $\mathrm{l}$ :

$$
\rho_{\mathrm{P}, \mathrm{Q}}^{\mathrm{tme}}(l) \leq 2 \cdot \rho_{\mathrm{Q}, \mathrm{P}}^{\mathrm{tme}}(l)-1
$$

Proof. By Corollary 7.14, there is a trace $t \in B^{l}$, such that

$$
\frac{\rho_{Q, \mathrm{P}}^{\mathrm{tme}}(l)-1}{2} \leq\left|\mathrm{Q}_{\mathrm{tme}}(t)-\mathrm{P}_{\mathrm{tme}}(t)\right|=\left|\mathrm{P}_{\mathrm{tme}}(t)-Q_{\mathrm{tme}}(t)\right| \leq \rho_{\mathrm{P}, \mathrm{t}}^{\mathrm{tme}}(l)-1 .
$$

Multiplying both sides by 2 and adding 1 finish the proof. 


\subsubsection{A Linear Lower Bound on the Leak Ratio}

In this section, we show that if the difference in misses between two cache algorithms is unbounded, then there are traces on which the difference in misses grows linearly in the length of the trace. Together with the result from the previous section, this implies that the leak ratio between two algorithms grows linearly in the length of the trace if and only if the difference between the two algorithms is unbounded. This result does not hold for arbitrary caches conforming to the model introduced in Chapter 2. We need to make two additional assumptions:

1. We assume the set of control states $S_{\mathrm{p}}$ of a cache algorithm to be finite. This is naturally the case for hardware-based caches that maintain a finite set of status bits to guide future eviction decisions.

2. We assume that the evict function, evict $S_{\mathrm{P}} S_{\mathrm{P}} \times B \rightarrow S_{\mathrm{P}} \times\left\{0, \ldots, \eta_{\mathrm{P}}-1\right\}$ is independent of its second parameter, i.e., $\operatorname{evict}_{\mathrm{P}}(s, b)=\operatorname{evict}_{\mathrm{P}}\left(s, b^{\prime}\right)$ for all $s \in S_{\mathrm{P}}$ and $b, b^{\prime} \in B$. This assumption is naturally fulfilled by fully-associative caches, where there is no restriction on the placement of a memory block based on its address. This assumption could be significantly weakened at the expense of a more complicated proof. ${ }^{1}$

For the proof of the result we argue that, while there is an unbounded number of different cache configurations, even assuming an unbounded supply of memory blocks $B$, there are only finitely many "non-congruent" pairs of cache configurations, where congruent will be defined precisely below. Intuitively, congruent pairs of cache configurations behave similarly to each other, if their cache contents are appropriately renamed.

Such a renaming can be captured by a bijection. Let $\pi: B \rightarrow B$ be a bijection on memory blocks and let $\pi^{*}$ denote its extension to cache contents that maps $\perp$ to $\perp$ :

$$
\pi^{*}(c)=\lambda l \cdot \begin{cases}\pi(c(l)) & : c(l) \in B \\ \perp & : c(l)=\perp\end{cases}
$$

We also lift $\pi$ to cache configurations with $\pi^{*}(s, c)=\left(s, \pi^{*}(c)\right)$ and to traces with $\pi^{*}(\epsilon)=\epsilon$ and $\pi^{*}(b \circ t)=\pi(b) \circ \pi^{*}(t)$.

Let $(s, c)$ be an arbitrary cache configuration. Observe that:

$$
\forall t \in B^{*}: \pi^{*}\left(u p d_{\mathrm{P}}((s, c), t)\right)=\operatorname{upd}_{\mathrm{p}}\left(\pi^{*}(s, c), \pi^{*}(t)\right),
$$

i.e. renamed cache configurations behave the same on renamed accesses. Also observe that:

$$
\mathrm{P}_{\mathrm{tme}}(c, b,)=\mathrm{P}_{\mathrm{tme}}\left(\pi^{*}(c), \pi(b)\right),
$$

\footnotetext{
${ }^{1} \mathrm{~A}$ weaker, yet sufficient condition would be that there is a finite partition of $B$, such that $\operatorname{evict}_{\mathrm{P}}(s, b)=\operatorname{evict}_{\mathrm{P}}\left(s, b^{\prime}\right)$ for all $s \in S_{\mathrm{P}}$ and $b, b^{\prime}$ that are in the same block of the partition. This weaker assumption is fulfilled by arbitrary set-associative caches.
} 
which holds because $\pi(b)$ is contained in $\pi^{*}(c)$ if and only if $b$ is contained in $c$. From these two observations, it follows that:

$$
\mathrm{P}_{\text {tme }}((s, c), t)=\mathrm{P}_{\text {tme }}\left(\pi^{*}(s, c), \pi(t)\right) .
$$

Definition 7.16 (Congruent cache configurations). Two pairs of cache configurations $\left(g_{\mathrm{P}}, g_{Q}\right)$ and $\left(g_{\mathrm{P}}^{\prime}, g_{0}^{\prime}\right)$ are congruent, denoted by $\left.\left(g_{\mathrm{P}}, g_{0}\right)\right) \equiv\left(g_{\mathrm{P}}^{\prime}, g_{Q}^{\prime}\right)$, if there is a bijection $\pi: B \rightarrow B$, such that $g_{\mathrm{P}}^{\prime}=\pi^{*}\left(g_{\mathrm{P}}\right)$ and $g_{0}^{\prime}=\pi^{*}\left(g_{0}\right)$. To indicate a bijection $\pi$ that is a witness to the congruence of two pairs of cache configurations we also write $\left(g_{\mathrm{P}}, g_{0}\right) \equiv_{\pi}\left(g_{\mathrm{P}}^{\prime}, g_{0}^{\prime}\right)$.

Note that congruence is an equivalence relation. We denote the equivalence class of a pair of cache configuration $\left(g_{\mathrm{P}}, g_{0}\right)$ by

$$
\left[g_{\mathrm{P}}, g_{Q}\right]:=\left\{\left(g_{\mathrm{P}}^{\prime}, g_{Q}^{\prime}\right) \in G_{\mathrm{P}} \times G_{Q} \mid\left(g_{\mathrm{P}}^{\prime}, g_{Q}^{\prime}\right) \equiv\left(g_{\mathrm{P}}, g_{Q}\right)\right\} .
$$

While the set of pairs of cache configurations is infinite, its quotient w.r.t. to the congruence relation is finite:

Theorem 7.17 (Index of $\equiv$ ). Let $\mathrm{P}$ and $\mathrm{Q}$ be two finite-control-state cache algorithms. Then, the quotient

$$
G_{\mathrm{P}} \times G_{0} / \equiv=\left\{\left[g_{\mathrm{P}}, g_{0}\right] \mid\left(g_{\mathrm{P}}, g_{0}\right) \in G_{\mathrm{P}} \times G_{0}\right\}
$$

is finite.

Proof. Remember that $\eta_{\mathrm{P}}$ and $\eta_{\mathrm{Q}}$ denote the capacities of $\mathrm{P}$ and $\mathrm{Q}$. Let $B_{\mathrm{P}, \mathrm{Q}}$ be an arbitrary but fixed subset of $B$, such that $\left|B_{\mathrm{P}, 0}\right|=\eta_{\mathrm{P}}+\eta_{\mathrm{Q}}$.

We show below that each pair $\left(\left(s_{P}, c_{P}\right),\left(s_{0}, c_{Q}\right)\right)$ of cache configurations is congruent to a pair of cache configurations $\left(\left(s_{\mathrm{P}}, c_{\mathrm{P}}^{\prime}\right),\left(s_{0}, c_{0}^{\prime}\right)\right)$ in which only blocks from $B_{\mathrm{P}, \mathrm{Q}}$ may occur in the cache contents $c_{\mathrm{P}}^{\prime}$ and $c_{0}^{\prime}$. As $B_{\mathrm{P}, \mathrm{Q}}$ is finite, there are only finitely many different cache contents $c_{\mathrm{P}}^{\prime}$ and $c_{0}^{\prime}$ containing only blocks from $B_{\mathrm{P}, \mathrm{Q}}$. The sets of control states $S_{\mathrm{P}}$ and $S_{\mathrm{Q}}$ are finite by assumption. Together, this implies that the set of equivalence classes of $\equiv$ is finite.

Below we show how to incrementally construct a bijection $\pi: B \rightarrow B$ such that the contents of $c_{\mathrm{P}}^{\prime}=\pi^{*}\left(c_{\mathrm{P}}\right)$ and $c_{\mathrm{Q}}^{\prime}=\pi^{*}\left(c_{\mathrm{Q}}\right)$ contain only blocks from $B_{\mathrm{P}, \mathrm{Q}}$ :

1. Initially, let $\pi$ be the identity function on $B$, and let $D=B_{\mathrm{P}, \mathrm{Q}}$.

2. For $i=0, \ldots, \eta_{\mathrm{P}}-1$ :

If $c_{\mathrm{P}}(i) \in B_{\mathrm{P}, \mathrm{Q}}$ then modify $D$ to $D=D \backslash\left\{c_{\mathrm{P}}(i)\right\}$.

3. For $j=0, \ldots, \eta_{0}-1$ :

If $c_{Q}(j) \in B_{\mathrm{P}, Q}$ then modify $D$ to $D=D \backslash\left\{c_{Q}(j)\right\}$.

4. For $i=0, \ldots, \eta_{\mathrm{P}}-1$ :

If $c_{\mathrm{P}}(i) \neq \perp$ and $\pi\left(c_{\mathrm{P}}(i)\right) \notin B_{\mathrm{P}, 0}$, then pick $b \in D$ and modify $\pi$ and $D$ as follows:

$\pi=\pi\left[c_{\mathrm{P}}(i) \mapsto b\right]\left[b \mapsto c_{\mathrm{P}}(i)\right]$ and $D=D \backslash\{b\}$ 
5. For $j=0, \ldots, \eta_{0}-1$ :

If $c_{0}(j) \neq \perp$ and $\pi\left(c_{0}(j)\right) \notin B_{\mathrm{P}, Q}$, then pick $b \in D$ and modify $\pi$ and $D$ as follows:

$$
\pi=\pi\left[c_{0}(j) \mapsto b\right]\left[b \mapsto c_{0}(j)\right] \text { and } D=D \backslash\{b\} .
$$

Note that there is always a $b \in D$ available, when the above algorithm needs one, because the operation is applied at most $\left|B_{\mathrm{P}, \mathrm{Q}}\right|=\eta_{\mathrm{P}}+\eta_{\mathrm{Q}}$ times. Throughout its execution, the algorithm maintains the invariant that $\pi$ is a bijection. Further, the resulting bijection satisfies $\pi^{*}\left(c_{\mathrm{P}}\right), \pi^{*}\left(c_{Q}\right) \subseteq B_{\mathrm{P}, 0} \cup\{\perp\}$.

We can exploit Theorem 7.17 in a manner similar to the application of the pumping lemma for regular languages in the proof of the following theorem.

Theorem 7.18. Let $\mathrm{P}$ and $\mathrm{Q}$ be two finite-control-state cache algorithms. Further, let the difference in misses between $\mathrm{P}$ and $\mathrm{Q}$ be unbounded, i.e.,

$$
\forall m \in \mathbb{N}: \exists t \in B^{*}:\left|P_{\text {tme }}(t)-Q_{\text {tme }}(t)\right|>m .
$$

Then, there is an $f \in \mathbb{R}, f>0$ and an $m_{0} \in \mathbb{N}$, such that

$$
\forall m \in \mathbb{N}, m>m_{0}: \exists t \in B^{m}:\left|\mathrm{P}_{\mathrm{tme}}(t)-Q_{\mathrm{tme}}(t)\right|>f \cdot|t| .
$$

Proof. Notation: We use $\mathrm{P}_{\text {tme }}\left(t, t^{\prime}\right)$ is a shortcut for $\mathrm{P}_{\text {tme }}\left(t t^{\prime}\right)-\mathrm{P}_{\text {tme }}(t)$, i.e., the number of misses on the suffix $t^{\prime}$.

Let $\mathrm{P}$ and $\mathrm{Q}$ be two finite-control-state cache algorithms such that the difference in misses between $P$ and $Q$ is unbounded. Let $l=\left|G_{P} \times G_{Q} / \equiv\right|+1$, which must be finite due to Theorem 7.17.

As the difference in misses between $P$ and $Q$ is unbounded, there must be a $t \in B^{*}$ such that $\left|\mathrm{P}_{\text {tme }}(t)-Q_{\text {tme }}(t)\right|=l$. We will assume without loss of generality ${ }^{2}$ that $P_{\text {tme }}(t)>Q_{\text {tme }}(t)$ for such traces $t$, and so $\left|P_{\text {tme }}(t)-Q_{\text {tme }}(t)\right|=P_{\text {tme }}(t)-Q_{\text {tme }}(t)$. Then, let $t_{1}, \ldots, t_{l}$ be prefixes of $t$, s.t. $P_{\text {tme }}\left(t_{j}\right)-Q_{\text {tme }}\left(t_{j}\right)=j$ for all $1 \leq j \leq l$.

Let $i g_{\mathrm{P}}=\left(i_{\mathrm{P}}, \lambda j . \perp\right)$ and $i g_{0}=\left(i_{0}, \lambda j . \perp\right)$ be the initial configurations of $\mathrm{P}$ and Q. Also, let $p_{j}=u p d_{\mathrm{P}}\left(i g_{\mathrm{P}}, t_{j}\right)$ and $q_{j}=u p d_{0}\left(i g_{0}, t_{j}\right)$ for all $1 \leq j \leq l$.

Due to the pigeonhole principle, there must be at least two prefixes $t_{j}$ and $t_{k}$, with $j<k$, such that the pairs of cache configurations $\left(p_{j}, q_{j}\right)$ and $\left(p_{k}, q_{k}\right)$ resulting from executing these prefixes are congruent. Assume that $t_{j}$ and $t_{k}$ are two such prefixes.

As $t_{j}$ is a prefix of $t_{k}$, we can decompose $t_{k}$ into $t_{j}$ and $t_{j \rightarrow k}$, such that $t_{k}=t_{j}$ o $t_{j \rightarrow k}$. From $\mathrm{P}_{\text {tme }}\left(t_{j}\right)-Q_{\text {tme }}\left(t_{j}\right)=j$ and $\mathrm{P}_{\text {tme }}\left(t_{k}\right)-Q_{\text {tme }}\left(t_{k}\right)=k$ we can conclude that $\mathrm{P}_{\mathrm{tme}}\left(t_{j}, t_{j \rightarrow k}\right)-Q_{\mathrm{tme}}\left(t_{j}, t_{j \rightarrow k}\right)=\left(\mathrm{P}_{\mathrm{tme}}\left(t_{k}\right)-\mathrm{P}_{\mathrm{tme}}\left(t_{j}\right)\right)-\left(Q_{\mathrm{tme}}\left(t_{k}\right)-Q_{\mathrm{tme}}\left(t_{j}\right)\right)=k-j \geq 1$.

\footnotetext{
${ }^{2}$ If $\mathrm{P}_{\text {tme }}(t)<Q_{\text {tme }}(t)$ the following arguments hold with $\mathrm{P}$ and $\mathrm{Q}$ exchanged.
} 
We can arbitrarily extend $t_{j}$ using the following construction of the traces $\tau_{m}$ and $\omega_{m}$ :

$$
\begin{aligned}
\tau_{0} & =t_{j}, \\
\tau_{m+1} & =\tau_{m} \circ \omega_{m}, \\
\omega_{0} & =t_{j \rightarrow k}, \\
\omega_{m+1} & =\pi^{*}\left(\omega_{m}\right) .
\end{aligned}
$$

Let $u_{m}=u p d_{\mathrm{P}}\left(i g_{\mathrm{P}}, \tau_{m}\right)$ and $v_{m}=u p d_{0}\left(i g_{0}, \tau_{m}\right)$. For the following induction proof, it will be helpful to express $u_{m+1}$ and $v_{m+1}$ in terms of $u_{m}$ and $v_{m}$. We have that $u_{m+1}=u p d_{\mathrm{P}}\left(i g_{\mathrm{P}}, \tau_{m} \circ \omega_{m}\right)=\operatorname{upd}_{\mathrm{P}}\left(u p d_{\mathrm{P}}\left(i g_{\mathrm{P}}, \tau_{m}\right), \omega_{m}\right)=u p d_{\mathrm{P}}\left(u_{m}, \omega_{m}\right)$ and similarly $v_{m+1}=u p d_{0}\left(v_{m}, \omega_{m}\right)$.

We show by induction that $\left(u_{m}, v_{m}\right) \equiv_{\pi}\left(u_{m+1}, v_{m+1}\right)$ :

- (Induction base) For $m=0, \tau_{0}=t_{j}$ and $\tau_{1}=\tau_{0} \circ \omega_{0}=t_{j} \circ t_{j \rightarrow k}=t_{k}$. Thus we have that $u_{0}=u p d_{\mathrm{P}}\left(i g_{\mathrm{P}}, \tau_{0}\right)=u p d_{\mathrm{P}}\left(i g_{\mathrm{P}}, t_{j}\right)=p_{j}$ and $v_{0}=u p d_{0}\left(i g_{0}, \tau_{0}\right)=$ $\operatorname{upd}_{0}\left(i g_{0}, t_{j}\right)=q_{j}$. Similarly, $u_{1}=p_{k}$ and $v_{1}=q_{k}$, and we already know that $\left(p_{j}, q_{j}\right) \equiv_{\pi}\left(p_{k}, q_{k}\right)$.

- (Induction step) For $m>0$, we know from the induction hypothesis that $\left(u_{m-1}, v_{m-1}\right) \equiv_{\pi}\left(u_{m}, v_{m}\right)$. Applying (7.6) with $t=\omega_{m-1}$ yields $\pi^{*}\left(u_{m}\right)=$ $\pi^{*}\left(\operatorname{upd}_{\mathrm{P}}\left(u_{m-1}, \omega_{m-1}\right)\right)=\operatorname{upd}_{\mathrm{P}}\left(u_{m}, \pi^{*}\left(\omega_{m-1}\right)\right)=\operatorname{updP}\left(u_{m}, \omega_{m}\right)=u_{m+1}$, and similarly $\pi^{*}\left(v_{m}\right)=v_{m+1}$. Thus $\left(u_{m}, v_{m}\right) \equiv_{\pi}\left(u_{m+1}, v_{m+1}\right)$.

Since we have that $u_{m+1}=\pi\left(u_{m}\right)$ and $v_{m+1}=\pi\left(v_{m}\right)$, applying (7.8) yields $\mathrm{P}_{\text {tme }}\left(u_{m+1}, \omega_{m+1}\right)=\mathrm{P}_{\text {tme }}\left(u_{m+1}, \pi^{*}\left(\omega_{m}\right)\right)=\mathrm{P}_{\text {tme }}\left(u_{m}, \omega_{m}\right)$ and similarly we have $\mathrm{P}_{\text {tme }}\left(v_{m+1}, \omega_{m+1}\right)=\mathrm{P}_{\text {tme }}\left(v_{m}, \omega_{m}\right)$ for all $m$. In other words, the number of misses on the subtraces $\omega_{m}$ are always the same in both $P$ and $Q$. We also know that $\mathrm{P}_{\mathrm{tme}}\left(u_{0}, \omega_{0}\right)=\mathrm{P}_{\mathrm{tme}}\left(t_{j}, t_{j \rightarrow k}\right)$ and $\mathrm{Q}_{\mathrm{tme}}\left(v_{0}, \omega_{0}\right)=Q_{\mathrm{tme}}\left(t_{j}, t_{j \rightarrow k}\right)$. Thus, we have

$$
\begin{aligned}
\mathrm{P}_{\mathrm{tme}}\left(\tau_{m}\right) & =\mathrm{P}_{\mathrm{tme}}\left(t_{j}\right)+m \cdot \mathrm{P}_{\mathrm{tme}}\left(t_{j}, t_{j \rightarrow k}\right), \\
Q_{\mathrm{tme}}\left(\tau_{m}\right) & =Q_{\mathrm{tme}}\left(t_{j}\right)+m \cdot Q_{\mathrm{tme}}\left(t_{j}, t_{j \rightarrow k}\right), \\
\mathrm{P}_{\mathrm{tme}}\left(\tau_{m}\right)-Q_{\mathrm{tme}}\left(\tau_{m}\right) & =\mathrm{P}_{\mathrm{tme}}\left(t_{j}\right)-Q_{\mathrm{tme}}\left(t_{j}\right)+m \cdot\left(\mathrm{P}_{\mathrm{tme}}\left(t_{j}, t_{j \rightarrow k}\right)-Q_{\mathrm{tme}}\left(t_{j}, t_{j \rightarrow k}\right)\right), \\
& =\mathrm{P}_{\mathrm{tme}}\left(t_{j}\right)-Q_{\mathrm{tme}}\left(t_{j}\right)+m \cdot(k-j) .
\end{aligned}
$$

Let $f=\frac{k-j}{\left|t_{j \rightarrow k}\right|+1} \geq \frac{1}{\left|t_{j \rightarrow k}\right|+1}>0$. For large enough $m$,

$$
\left|\mathrm{P}_{\mathrm{tme}}\left(\tau_{m}\right)-Q_{\mathrm{tme}}\left(\tau_{m}\right)\right|=\mathrm{P}_{\mathrm{tme}}\left(t_{j}\right)-Q_{\mathrm{tme}}\left(t_{j}\right)+m \cdot(k-j),
$$

is greater than $f \cdot\left|\tau_{m}\right|=\frac{k-j}{\left|t_{j \rightarrow k}\right|+1} \cdot\left(\left|\tau_{0}\right|+m \cdot\left|t_{j \rightarrow k}\right|\right)$, which proves the theorem.

In other words, if the difference in misses between two finite-control-state algorithms is unbounded, then it actually grows linearly in the length of the trace. 
Theorem 7.19. The leak ratio between two finite-control-state cache algorithms $P$ and $\mathrm{Q}$ grows linearly in the length of the trace if and only if the difference in misses between $\mathrm{P}$ and $\mathrm{Q}$ is unbounded:

$$
\rho_{\mathrm{P}, \mathrm{Q}}^{\mathrm{tme}}(l), \rho_{\mathrm{Q}, \mathrm{P}}^{\mathrm{tme}}(l) \in \Omega(l) \quad \Leftrightarrow \quad \forall m \in \mathbb{N}: \exists t \in B^{*}:\left|\mathrm{P}_{\mathrm{tme}}(t)-Q_{\mathrm{tme}}(t)\right|>m .
$$

Proof. Direction " $\Rightarrow$ ": Assume for a contradiction that there is an $m_{\max } \in \mathbb{N}$, such that for all traces $t \in B^{*}:\left|P_{\text {tme }}(t)-Q_{\text {tme }}(t)\right| \leq m_{\text {max }}$. As $\rho_{\mathrm{P}, 0}^{\text {tme }}(l) \in \Omega(l)$ there must be an $l^{*}$, such that $\rho_{\mathrm{P}, 0}^{\mathrm{tme}}\left(l^{*}\right)>2 \cdot m_{\max }+1$. By the second part of Corollary 7.14 , there is a trace $t$ such that

$$
m_{\max }<\frac{\rho_{\mathrm{P}, 0}^{\mathrm{tme}}\left(l^{*}\right)-1}{2} \leq\left|\mathrm{P}_{\mathrm{tme}}(t)-Q_{\mathrm{tme}}(t)\right|
$$

which contradicts our assumption.

Direction " $\Leftarrow$ ": We will prove that $\rho_{\mathrm{P}, 0}^{\mathrm{tme}}(l) \in \Omega(l)$. The fact that $\rho_{0, \mathrm{P}}^{\mathrm{tme}}(l) \in$ $\Omega(l)$ follows by simply exchanging $P$ and $Q$ because $\left|P_{\text {tme }}(t)-Q_{\text {tme }}(t)\right|=\mid Q_{\text {tme }}(t)-$ $\mathrm{P}_{\text {tme }}(t) \mid$.

To prove that $\rho_{\mathrm{P}, 0}^{\mathrm{tme}}(l) \in \Omega(l)$, we have to show that there is a $k>0$ and an $m_{0} \in \mathbb{N}$, such that $\forall m \in \mathbb{N}, m>m_{0}: \rho_{\mathrm{P}, 0}^{\mathrm{tme}}(m) \geq k \cdot m$.

By Theorem 7.18, we can conclude that there is an $f>0$ and an $m_{0}^{\prime} \in \mathbb{N}$, such that $\forall m \in \mathbb{N}, m>m_{0}^{\prime}: \exists t \in B^{m}:\left|P_{\text {tme }}(t)-Q_{\text {tme }}(t)\right|>f \cdot|t|$. Pick $k$ to be $f$ and $m_{0}$ to be $m_{0}^{\prime}$. To prove the theorem it then remains to show that $\exists t \in B^{m}$ : $\left|\mathrm{P}_{\text {tme }}(t)-Q_{\text {tme }}(t)\right|>f \cdot|t|$ implies $\rho_{\mathrm{P}, 0}^{\mathrm{tme}}(m)>f \cdot m$.

To this end, let $t \in B^{m}$ be a trace that satisfies $\left|\mathrm{P}_{\text {tme }}(t)-Q_{\text {tme }}(t)\right|>f \cdot|t|$. Applying the first part of Corollary 7.14 yields $f \cdot|t|<\left|\mathrm{P}_{\mathrm{tme}}(t)-Q_{\mathrm{tme}}(t)\right|<\rho_{\mathrm{P}, \mathrm{Q}}^{\mathrm{tme}}(m)$.

\subsection{Access-based Attacker}

The access-based attacker obtains information not about the effect of the victim's program on the cache but about the final configuration of the cache after the victim's program has finished. This process is not as simple as directly observing the cache but actually requires that the attacker probes the cache, that is, accesses new memory blocks, observes the effect they have on the cache and draws conclusions about the configuration left by the victim.

When considering competitiveness analysis, our objective is to compare the leakage of two cache algorithms, not how secure they are individually. For that reason, we focus on the final leakage the attacker obtains from the cache, that is, the information extraction presented in Section 4.2.

As with Section 5.2, we focus on permutation-based cache algorithms, see Section 2.2. As we did when defining information absorption for the permutation-based model, Section 5.2.1, we abstract from programs using blocks from $B$ to the set of all possible traces using those blocks, $B^{*}$. Moreover, since only 
the size of the set of memory blocks is relevant, we define leak-competitiveness for an access-based attacker with a function depending on the footprint.

Definition 7.20. For a function $\rho^{\text {acc }}: \mathbb{N} \rightarrow \mathbb{R}_{\geq 0}$, we say that algorithm $P$ is $\rho^{\text {acc }}$ leak-competitive relative to algorithm $Q$ for an access-based attacker if, for all $f p \in \mathbb{N}$,

$$
\mu(\mathrm{P}, T) \leq \rho^{\mathrm{acc}}(f p) \cdot \mu(\mathrm{Q}, T),
$$

for all set of traces of blocks $T \subseteq B^{*}$ with $|B|=f p$ where $\mu(\mathrm{P}, T)$ denotes the maximum information leakage obtained from the set of configurations reachable from the initial configuration $\widehat{i g}_{\mathrm{P}}$ by the set of traces $T$.

As was the case with the time-based attacker, there always exists a value $\rho^{\operatorname{acc}}(f p)$ such that two cache algorithms are $\rho^{\text {acc }}(f p)$-leak-competitive. We then focus on the shape the leak-competitiveness relation takes as we modify the footprint of the set of memory blocks. For that we define the leak ratio for an access-based attacker.

Definition 7.21. Given a pair of algorithms $\mathrm{P}$ and $\mathrm{Q}$ we define the leak ratio for an access-based attacker $\rho_{\mathrm{P}, 0}^{\mathrm{acc}}$ as:

$\rho_{\mathrm{P}, Q}^{\mathrm{acc}}(f p)=\min \left\{\rho^{\mathrm{acc}}(f p) \mid \rho^{\mathrm{acc}}: \mathbb{N} \rightarrow \mathbb{R}_{\geq 0}, \mathrm{P}\right.$ is $\rho^{\text {acc }}$-leak-competitive relative to $\left.Q\right\}$.

For the competitiveness analysis we always assume that, before the victim accesses it, the cache starts in the initial configuration, $\widehat{i g}_{P}$, that is, an empty configuration. We do not need to specify the analysis to the configuration since we will look at the asymptotic behavior of $\rho_{\mathrm{P}, 0}^{\mathrm{acc}}$ and the results obtained in Section 5.2.2 are independent of the configuration of the cache prior to the victim's access.

We now look at the results on information extraction obtained for LRU, FIFO and PLRU on Section 5.2.2 and interpret them in terms of the leak ratio for an access-based attacker:

- For FIFO and LRU with shared memory, the number of observations of an access-based attacker is bounded by a constant, Proposition 5.17. This implies that the leak ratios of FIFO relative to LRU, and of LRU relative to FIFO, are in $\mathcal{O}(1)$.

- For PLRU with shared memory and associativity bigger than two, the number of observations grows with the footprint, Proposition 5.18, and this growth is linear in the case of associativity four, Figures 5.2e and 5.2f. This implies that the leak ratio of PLRU with associativity bigger than two relative to FIFO and LRU respectively, is in $\Omega(n)$, whereas the leak ratio of FIFO and LRU relative to PLRU. This is an example of different competitiveness relationships. 
- For LRU, FIFO and PLRU with a disjoint memory attacker, the number of observations of an access-based attacker is bounded by a constant, Propositions 5.19 and 5.20. This implies that the leak ratios of any pair of cache algorithms, for a disjoint memory attacker, are in $\mathcal{O}(1)$.

Overall, these examples show that, unlike for time-based attackers, there are dominance relations for the security of cache algorithms with respect to accessbased attackers. We leave a detailed investigation of this case to future work. 


\section{CHAPTER 8}

\section{RELATED WORK}

\section{Quantitative information-flow analysis}

The notion of leakage we use is based on concepts from quantitative informationflow analysis widely used in previous work. [CHM07] uses information theory, particularly Shannon's entropy, to measure interference between variables in imperative languages. [Smi09] explores the use of vulnerability and minentropy as a more realistic way of characterizing side channels. [ACPS12b] studies g-leakage, a generalization of min-entropy, that uses gain functions to allow for more accurate quantifications of leakage for specific scenarios.

These notions of information-flow analysis have been successfully used for detecting and quantifying side channels of program code: [HM10] analyses several vulnerabilities in the Linux Kernel and a couple of authentication routines, [NMS09] uses channel capacity measurement to improve the results of dynamic taint analysis, [KB07] quantifies the information that an adaptive attacker can extract under different notions of entropy and [DKMR15] uses the notion of vulnerability to give upper bounds on the security of cache sidechannel attacks.

\section{Other works on the security of caches}

Our model of the access-based attacker is a refinement of the model proposed in [DKMR15]. This work assumes that the attacker can obtain information about the configuration of the cache left by the victim by directly observing it. In reality, observing the cache is not possible and the only way to obtain information about its configuration is by accessing new memory blocks and observing whether they produce a hit or a miss, a process we refer to as probing the cache. This refinement of the model allows to obtain better security guarantees at the expense of having to compute an optimal probing strategy. 
When it comes to timing attacks, a simple way to reduce the vulnerability to leak information is introducing some sort of randomness in the replacement of memory blocks, this has been proposed by several authors, see [HL17, $\left.\mathrm{KCQ}^{+} 13, \mathrm{ZL} 14\right]$. With deterministic cache algorithms, the observation that the attacker obtains only depends on the victim's accesses to memory and thus there is no noise interfering with the attacker's guess.

For the access-based attacker, one way to mitigate the leakage is by partitioning the cache lines in disjoint subsets, one for each user [HL17, ZL14]. This way, attacker's memory blocks do not compete with victim's memory blocks for cache lines which denies the posibility of probing the cache.

Our work considers cache algorithms that neither use any randomness in the replacement nor partition the cache between users, acknowledges that leaking information is unavoidable for these types of cache algorithms and explores how different cache algorithms make the leakage less dangerous.

\section{Work related to information extraction}

Our definition of a probing strategy is made with the notion that the attacker probes the cache in an adaptive way, that is, reacting to the effect previously accessed memory blocks have on the cache. This is related related to existing models for adaptive probing [KB07, BP12]. There, however, the secret remains static. The model of [KB07] and the deterministic part of [BP12] is a special case of ours, where accesses to memory blocks do not modify the configuration of the cache left by the victim.

Mardziel et al. [MAHC14] develop an approach to quantify information flow for dynamic secrets, that is, secrets that evolve over time. They consider a probabilistic system and attacks that consist of a fixed amount of steps. Attacks finish with an exploit whose success is evaluated using gain functions [ACPS12b]. Our model for information extraction differs from their model in that it is deterministic and allows to compute leakage for an undetermined number of attacks steps, i.e., until the probe is depleted. We further provide an algorithm that actually allows us to compute optimal strategies.

The problem of computing the information extraction is related to the state-identification problem for Mealy machines, which was first introduced by Moore in [Moo56], expanded upon by Gill in [Gil61], and analyzed from a complexity perspective by Lee and Yannakakis [LY94]. The state-identification problem is to determine the initial state of a Mealy machine by probing strategies, just as in our case. While we are interested in the maximal number of knowledge sets into which the uncertainty about the initial state can be partitioned, state-identification algorithms are only concerned with the decision problem, that is whether or not a full identification, i.e, a partitioning into singleton knowledge sets is feasible, and if it is, by which strategy. So our problem of finding the finest partition can be seen as a quantitative generalization of the 
state-identification problem.

\section{Original notions of competitiveness}

Leak competitiveness is inspired by work on competitive performance analysis. The notion of competitive analysis was first introduced in [ST85], where the authors bound the number of misses an online algorithm does on a trace of memory blocks in terms of the number of misses of an optimal offline algorithm [Bel66]. In contrast to performance, there is no clear candidate for an optimal offline cache algorithm for security, because the best option would be not to cache memory blocks and be trivially non-interferent. This is why we base leak competitiveness on relative competitiveness [RG08]. Here, the number of misses one cache algorithm produces is compared with the number of misses of another algorithm, none of them necessarily optimal. 


\section{CHAPTER 9}

\section{CONCLUSIONS}

Although cache memories are a very important component of modern computers since they improve the efficiency of programs, they can also be used for malicious activities as the variations of execution time may provide information on secret data the program uses. This thesis focuses on the security aspects of cache side-channel attacks, specifically on the role the cache algorithm governing the cache plays in the security of the cache.

We consider two classes of cache algorithms: automata-based cache algorithms, that encompass a wide variety of cache algorithms, and permutationbased cache algorithms, a subclass of the automata-based cache algorithms that encompass common cache algorithms like Least Recently Used or First In First Out.

As a way for quantifying the security of the cache algorithm, we use notions of information theory and express the security guarantee of the side-channel as an upper bound on the probability of the attacker correctly guessing a secret chosen by the victim. This bound is incremented if the attacker has access to side-channel observations (the information leaked through the side-channel) and the increase depends on the number of different observations the attacker obtains.

With the two models for the cache algorithms and the notion of security as number of observations, we study the security of cache algorithms with respect to three common types of attackers: time-based, that observe the total number of misses a trace of memory blocks does, trace-based, that observe the outcome of every individual access of a trace of memory blocks, and access-based, that obtain partial information about the configuration of the cache left by a trace of memory blocks. 


\subsection{Contributions}

\section{Upper bounds on Leakage}

We derive program dependent and independent upper bounds on the leakage a cache algorithm may produce.

For the program dependent analysis, we improve current results by introducing a more realistic model of the access-based attacker. This model is based on the idea that the attacker has to probe the cache in order to know its content, thus modifying the configuration left by the victim. We introduce two notions to analyze the security of caches against the access-based attacker: how much information the cache absorbs from the victim's computation and how much information the attacker can extract from the cache.

We also introduce an algorithm that, given a cache algorithm and a set of victim's configurations, computes the value for the information extraction for that cache algorithm and set of configurations.

For the program independent analysis we use worst-case analysis to derive upper bounds on the number of observations for a cache algorithm for any set of traces of memory blocks. This allows to obtain security guarantees that only depend on the cache algorithm.

For the time and trace-based attackers we show that the effect a memory block produces on the cache only depends on whether the block is in the cache or not. Then, the worst-case values for leakage depend on how filled the cache is prior to the victim's computations, i.e. how many lines have a valid memory block, and the number of different memory blocks the victim uses, the footprint of the program. We conclude that all cache algorithm leak the same amount of information in their worst-case scenario.

For the access-based attacker we show that permutation-based cache algorithms are oblivious to the actual memory blocks in the cache, they update the configurations by doing equality checks with the accessed memory block and comparing the ages of the blocks. Then, the worst-case information absorption, and thus extraction, depend on how filled the cache is prior to the victim's computations, we focus on the extreme cases empty and completely filled, and on the number of different memory blocks the victim uses, the footprint of the program. For three common cache algorithms, LRU, FIFO and PLRU, we obtain results about their information extraction, either in the form of upper bounds (LRU and FIFO) or with results that show that the information extraction grows with the footprint (PLRU). We conclude that, for each combination of configuration of the cache and footprint, a different cache algorithm provides the lowest leakage, either because the information absorption is low (eg. FIFO with a filled cache and low footprint) or because the information extraction is low (eg. LRU with large footprint).

When considering set-associative caches and the access-based attacker we 
show that distributing the memory blocks over several cache sets does not necessarily mean lower leakage as the multiplicative effect of several cache sets may take over the decrease in information absorption per cache set.

We also implement our information extraction algorithm into the CacheAudit static analyzer to refine the security guarantees of the access-based attacker. We test our algorithm on several implementations of AES, Sosemanuk and $\mathrm{HC}-128$ and show a gain in precision of the analysis when considering information extraction rather than absorption.

We compare the upper bounds obtained with worst-case analysis and the computed ones returned by CacheAudit. With them we conclude that the leakage of AES computed by CacheAudit differs very little from the upper bounds (except for some cases where CacheAudit over approximates the set of reachable configurations), due to the very diverse access pattern of AES. However, the computed leakage for Sosemanuk and HC-128 differs significantly from the upper bounds since these cryptographic algorithms access memory in a less diverse way that reduces the information absorption.

\section{Competitiveness analysis}

Competitiveness analysis provides a comparison between cache algorithms by analyzing the largest difference in leakage between two cache algorithms. However, it is not suited to analyze the individual security of cache algorithms as the results are given relative to another algorithm.

For the time-based attacker we show that no cache algorithm dominates another in terms of leak ratio, that is, for each pair of cache algorithms $P, 0$ both leak ratios, $\rho_{\mathrm{P}, 0}^{\mathrm{tme}}$ and $\rho_{0, \mathrm{P}}^{\text {tme }}$ grow at the same rate. This implies that, for any set of traces of memory blocks where cache algorithm $P$ leaks less than $Q$, there exist a set where cache algorithm $Q$ leaks less than $P$ and both differences in leakage are comparable.

For the access-based attacker we use the upper bounds on leakage and show that some pairs of cache algorithms have comparable leak ratios and so there is no dominance of one over the other (eg. LRU and FIFO) and some pairs of cache algorithms have leak ratios that grow at different rates and so show a dominance of one algorithm over the other (eg. LRU dominates PLRU). 


\subsection{Future Work}

We now mention some possible future directions for the research developed in the thesis.

\section{Upper bounds on other cache algorithms}

The automata-based model encompasses a wide variety of cache algorithms and so the upper bounds obtained for the time and trace-based attacker are quite general. However, for the case of the access-based attacker we have developed upper bounds for only three permutation-based cache algorithms. A clear extension of these results would be to model more cache algorithms and obtaining similar upper bounds for the information extraction. This would require modeling the cache algorithm in a way that allows to compute a set of victim's configurations and then apply our probing algorithm.

\section{Specialized probing algorithm}

The probing algorithm proposed in Algorithm 1 is not a very efficient one but is able to compute the information extraction for any cache algorithm. We are aware of some modifications that could be made to specialize it to specific cache algorithms that could improve the computation. For example, for LRU, after a memory block has been used, it will always return a hit if repeated and thus, it does not need to be considered when extending the probe (line 7 of Algorithm 1).

\section{Competitiveness on sets of traces}

The non-dominance results for the time-based attacker that we have obtained on competitiveness analysis assume that any possible trace of memory blocks is possible. If we were to compare a pair of cache algorithms with respect to a program, we would restrict from all possible traces of memory blocks to a subset of traces. In that situation, dominance results for the time-based attacker could arise.

\section{Competitive analysis for the trace-based attacker}

We did some preliminary experiments on competitive analysis for the tracebased attacker and obtained a non-dominance result similar to Theorem 7.5 but that seemed to apply only on a specific class of automata-based cache algorithms. However, we were unable to characterizing this class by the time of writing the thesis. 


\section{BIBLIOGRAPHY}

[ACPS12a] Mário Alvim, Kostas Chatzikokolakis, Catuscia Palamidessi, and Geoffrey Smith. Measuring information leakage using generalized gain functions. In Computer Security Foundations Symposium (CSF), 2012 IEEE 25th, pages 265-279. IEEE, 2012.

[ACPS12b] Mário S Alvim, Konstantinos Chatzikokolakis, Catuscia Palamidessi, and Graeme Smith. Measuring information leakage using generalized gain functions. In CSF, pages 265-279. IEEE, 2012.

[AK06] Onur Acriçmez and Çetin Koç. Trace-driven cache attacks on AES. Information and Communications Security, pages 112-121, 2006.

[AMWH94] Robert D Arnold, Frank Mueller, David B Whalley, and Marion G Harmon. Bounding worst-case instruction cache performance. In RTSS, pages 172-181, 1994.

[AR13] Andreas Abel and Jan Reineke. Measurement-based modeling of the cache replacement policy. In RTAS, pages 65-74. IEEE, 2013.

[AS07] Aslan Askarov and Andrei Sabelfeld. Gradual release: Unifying declassification, encryption and key release policies. In SSP, pages 207-221. IEEE, 2007.

[ASK07] Onur Aciiçmez, Werner Schindler, and Çetin K Koç. Cache based remote timing attack on the AES. In CryptographersâĂŹ Track at the RSA Conference, pages 271-286. Springer, 2007.

[AZMM04] Hussein Al-Zoubi, Aleksandar Milenkovic, and Milena Milenkovic. Performance evaluation of cache replacement policies for the spec cpu2000 benchmark suite. In Proceedings 
of the 42nd annual Southeast regional conference, pages 267-272. ACM, 2004.

$\left[\mathrm{BBC}^{+} 08\right]$ Côme Berbain, Olivier Billet, Anne Canteaut, Nicolas Courtois, Henri Gilbert, Louis Goubin, Aline Gouget, Louis Granboulan, Cédric Lauradoux, Marine Minier, et al. Sosemanuk, a fast software-oriented stream cipher. In New stream cipher designs, pages 98-118. Springer, 2008.

[Bel66] Laszlo A. Belady. A study of replacement algorithms for a virtualstorage computer. IBM Systems journal, 5(2):78-101, 1966.

[Ber05] Daniel Bernstein. Cache-timing attacks on AES. http://cr.yp . to/antiforgery/cachetiming-20050414.pdf, 2005.

[BM06] Joseph Bonneau and Ilya Mironov. Cache-collision timing attacks against aes. In International Workshop on Cryptographic Hardware and Embedded Systems, pages 201-215. Springer, 2006.

[BP12] Michele Boreale and Francesca Pampaloni. Quantitative multirun security under active adversaries. In QEST. IEEE, 2012.

$\left[\mathrm{BZB}^{+}\right.$05] Guido Bertoni, Vittorio Zaccaria, Luca Breveglieri, Matteo Monchiero, and Gianluca Palermo. Aes power attack based on induced cache miss and countermeasure. In Information Technology: Coding and Computing, 2005. ITCC 2005. International Conference on, volume 1, pages 586-591. IEEE, 2005.

[CH01] Jason F Cantin and Mark D Hill. Cache performance for selected spec cpu 2000 benchmarks. ACM SIGARCH Computer Architecture News, 29(4):13-18, 2001.

[CHM07] David Clark, Sebastian Hunt, and Pasquale Malacaria. A static analysis for quantifying information flow in a simple imperative language. JCS, 15(3):321-371, 2007.

[CKR17] Pablo Cañones, Boris Köpf, and Jan Reineke. Security analysis of cache replacement policies. In POST, pages 189-209. Springer, 2017.

[CKR19] Pablo Cañones, Boris Köpf, and Jan Reineke. On the incomparability of cache algorithms in terms of timing leakage. Logical Methods in Computer Science, 15, 2019.

[CS10] Michael R. Clarkson and Fred B. Schneider. Hyperproperties. Journal of Computer Security, 18(6):1157-1210, 2010. 
[DKL ${ }^{+98] ~ J e a n-F r a n c o i s ~ D h e m, ~ F r a n c o i s ~ K o e u n e, ~ P h i l i p p e-A l e x a n d r e ~ L e r-~}$ oux, Patrick Mestré, Jean-Jacques Quisquater, and Jean-Louis Willems. A practical implementation of the timing attack. In International Conference on Smart Card Research and Advanced Applications, pages 167-182. Springer, 1998.

[DKMR15] Goran Doychev, Boris Köpf, Laurent Mauborgne, and Jan Reineke. CacheAudit: a tool for the static analysis of cache side channels. ACM Transactions on Information and System Security (TISSEC), 18(1):4:1-4:32, 2015.

[Dor10] Reza Dorrigiv. Alternative measures for the analysis of online algorithms, 2010.

[FKL ${ }^{+}$91] Amos Fiat, Richard M. Karp, Michael Luby, Lyle A. McGeoch, Daniel D. Sleator, and Neal E. Young. Competitive paging algorithms. Journal of Algorithms, 12(4):685-699, 1991.

[GBK11] David Gullasch, Endre Bangerter, and Stephan Krenn. Cache games - bringing access-based cache attacks on AES to practice. In SSP, pages 490-505. IEEE, 2011.

[Gil61] Arthur Gill. State-identification experiments in finite automata. Information and Control, 4(2-3):132-154, 1961.

[GRB ${ }^{+}$17] Ben Gras, Kaveh Razavi, Erik Bosman, Herbert Bos, and Christiano Giuffrida. Aslr on the line: Practical cache attacks on the mmu. NDSS (Feb. 2017), 2017.

[Gru12] Daniel Grund. Static Cache Analysis for Real-Time Systems: LRU, FIFO, PLRU. epubli, 2012.

[HL17] Zecheng He and Ruby B Lee. How secure is your cache against side-channel attacks? In Proceedings of the 50th Annual IEEE/ACM International Symposium on Microarchitecture, pages 341-353. ACM, 2017.

[HM10] Jonathan Heusser and Pasquale Malacaria. Quantifying information leaks in software. In ACSAC, pages 261-269. ACM, 2010.

[HP11] John L Hennessy and David A Patterson. Computer architecture: $a$ quantitative approach. Elsevier, 2011.

[KB07] Boris Köpf and David Basin. An Information-Theoretic Model for Adaptive Side-Channel Attacks. In CCS, pages 286-296. ACM, 2007. 
[KCQ $\left.{ }^{+} 13\right]$ Leonidas Kosmidis, Charlie Curtsinger, Eduardo Quiñones, Jaume Abella, Emery Berger, and Francisco J Cazorla. Probabilistic timing analysis on conventional cache designs. In Proceedings of the Conference on Design, Automation and Test in Europe, pages 603-606. EDA Consortium, 2013.

$\left[\mathrm{KGG}^{+} 18\right]$ Paul Kocher, Daniel Genkin, Daniel Gruss, Werner Haas, Mike Hamburg, Moritz Lipp, Stefan Mangard, Thomas Prescher, Michael Schwarz, and Yuval Yarom. Spectre attacks: Exploiting speculative execution. arXiv preprint arXiv:1801.01203, 2018.

[KQQ99] Francois Koeune, Jean-Jacques Quisquater, and Jean-Jacques Quisquater. A timing attack against rijndael. 1999.

[LSG ${ }^{+}$18] Moritz Lipp, Michael Schwarz, Daniel Gruss, Thomas Prescher, Werner Haas, Stefan Mangard, Paul Kocher, Daniel Genkin, Yuval Yarom, and Mike Hamburg. Meltdown. arXiv preprint arXiv:1801.01207, 2018.

[LWML16] Fangfei Liu, Hao Wu, Kenneth Mai, and Ruby B Lee. Newcache: Secure cache architecture thwarting cache side-channel attacks. IEEE Micro, 36(5):8-16, 2016.

[LY94] David Lee and Mihalis Yannakakis. Testing finite-state machines: State identification and verification. IEEE Transactions on computers, 43(3):306-320, 1994.

[LYG $\left.{ }^{+} 15\right] \quad$ Fangfei Liu, Yuval Yarom, Qian Ge, Gernot Heiser, and Ruby B. Lee. Last-level cache side-channel attacks are practical. In SSP, pages 605-622. IEEE, 2015.

[MAHC14] Piotr Mardziel, Mario S. Alvim, Michael Hicks, and Michael R. Clarkson. Quantifying information flow for dynamic secrets. In SSP, pages 540-555. IEEE, 2014.

[MM03] Nimrod Megiddo and Dharmendra S Modha. Arc: A self-tuning, low overhead replacement cache. In FAST, volume 3, pages 115130, 2003.

[Moo56] Edward F Moore. Gedanken-experiments on sequential machines. Automata studies, 34:129-153, 1956.

[MWS ${ }^{+}$17] Clémentine Maurice, Manuel Weber, Michael Schwarz, Lukas Giner, Daniel Gruss, Carlo Alberto Boano, Stefan Mangard, and Kay Römer. Hello from the other side: Ssh over robust cache covert channels in the cloud. NDSS, San Diego, CA, US, 2017. 
[NMS09] James Newsome, Stephen McCamant, and Dawn Song. Measuring channel capacity to distinguish undue influence. In PLAS, pages 73-85. ACM, 2009.

[OST06] Dag Arne Osvik, Adi Shamir, and Eran Tromer. Cache attacks and countermeasures: the case of AES. In CT-RSA, pages 1-20. Springer, 2006.

[Pag02] Dan Page. Theoretical use of cache memory as a cryptanalytic side-channel. IACR Cryptology ePrint Archive, 2002:169, 2002.

[QJP ${ }^{+}$07] Moinuddin K Qureshi, Aamer Jaleel, Yale N Patt, Simon C Steely, and Joel Emer. Adaptive insertion policies for high performance caching. In ACM SIGARCH Computer Architecture News, volume 35, pages 381-391. ACM, 2007.

[RG08] Jan Reineke and Daniel Grund. Relative competitive analysis of cache replacement policies. In LCTES, pages 51-60, New York, NY, USA, June 2008. ACM.

[Sch18] Felix Schröder. Security of cache replacement policies under sidechannel attacks. 2018.

[Sez93] André Seznec. A case for two-way skewed-associative caches. In ACM SIGARCH computer architecture news, volume 21, pages 169-178. ACM, 1993.

[Smi09] Geoffrey Smith. On the foundations of quantitative information flow. In FoSSaCS, pages 288-302. Springer, 2009.

[ST85] Daniel D Sleator and Robert E Tarjan. Amortized efficiency of list update and paging rules. Communications of the ACM, 28(2):202208, 1985.

[TSS ${ }^{+}$03] Yukiyasu Tsunoo, Teruo Saito, Tomoyasu Suzaki, Maki Shigeri, and Hiroshi Miyauchi. Cryptanalysis of des implemented on computers with cache. In International Workshop on Cryptographic Hardware and Embedded Systems, pages 62-76. Springer, 2003.

[WEE $\left.{ }^{+} 08\right]$ Reinhard Wilhelm, Jakob Engblom, Andreas Ermedahl, Niklas Holsti, Stephan Thesing, David Whalley, Guillem Bernat, Christian Ferdinand, Reinhold Heckmann, Tulika Mitra, et al. The worst-case execution-time problem - overview of methods and survey of tools. ACM Transactions on Embedded Computing Systems (TECS), 7(3):36, 2008. 
[Wil05] Reinhard Wilhelm. Determining bounds on execution times. Embedded Systems Handbook, 2, 2005.

[Wu08] Hongjun Wu. The stream cipher hc-128. In New stream cipher designs, pages 39-47. Springer, 2008.

[XDLB13] Xiaoya Xiang, Chen Ding, Hao Luo, and Bin Bao. HOTL: a higher order theory of locality. In ASPLOS, pages 343-356. ACM, 2013.

[YF14] Yuval Yarom and Katrina Falkner. FLUSH+RELOAD: A high resolution, low noise, L3 cache side-channel attack. In USENIX, pages 719-732. USENIX Association, 2014.

[ZL14] Tianwei Zhang and Ruby B Lee. New models of cache architectures characterizing information leakage from cache side channels. In Proceedings of the 30th Annual Computer Security Applications Conference, pages 96-105. ACM, 2014.

[ZW10] Xin-Jie Zhao and Tao Wang. Improved cache trace attack on AES and CLEFIA by considering cache miss and S-box misalignment. IACR Cryptology ePrint Archive, 2010:56, 2010. 


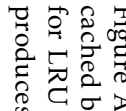

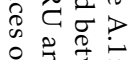

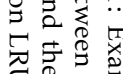

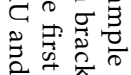

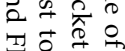

귕 क्ष

$\begin{array}{llll} & 1 & 0 & 0 \\ 0 & 0 & 0 \\ 0 & 0 & 0 \\ 0 & 0 & 0 \\ 0 & 0 & 0 \\ 2 & 0 & 0\end{array}$

: 20

छ

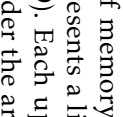

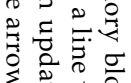

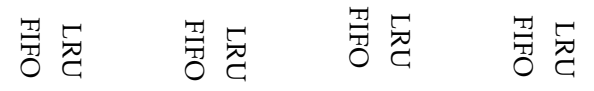

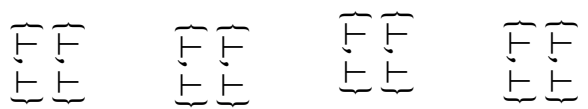

El

El>

SE

E上 E

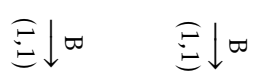

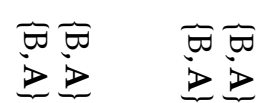

郃

$\tilde{\sigma} \bar{\nabla} \quad \tilde{\nabla}$

D西

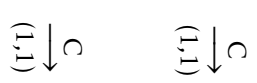

$\bar{\Omega} \quad \bar{\Omega}$

Ẽ

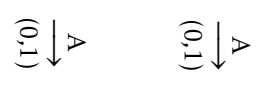

可

$\Omega \cong \quad \Omega \Omega$

就 豆收

实西西

$\cong \geq D$

PS

E上 $E$

总的导的

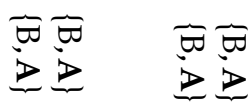

के

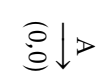

$\tilde{\sigma}_{\nabla}^{\infty} \quad \tilde{\nabla}$

Eln

Eln

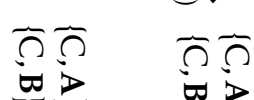

Elo

है।

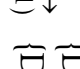

$\ddot{\theta} \quad \tilde{\sigma}$

E्ञ

Eld

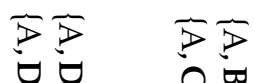

Elo Elo

$\bar{\omega} \bar{\omega}$

$\pm \Omega$

$\overline{0} \overline{0}$

잉ำ

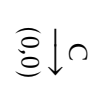

高柿

히긴

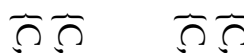

⿶凵⿴囗十)

El

还

Elo

ลิก

궘

$\ddot{\sigma}$

茰

인

总》

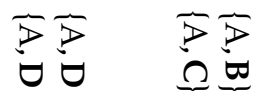

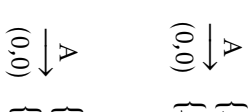

焉焉焉

$\begin{array}{ll}\ddot{G} & \ddot{G} \\ \text { v } & \text { e }\end{array}$

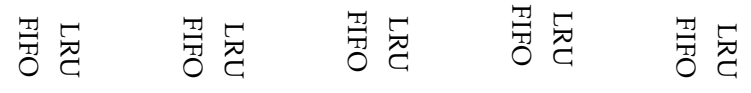

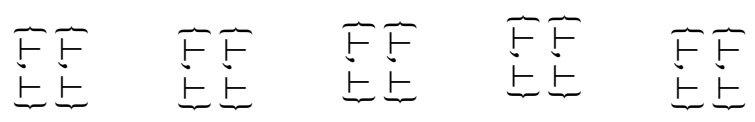

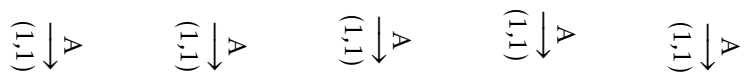

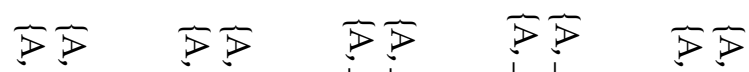

EE EE $5 E$ EE

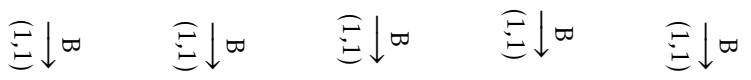

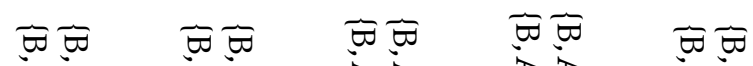

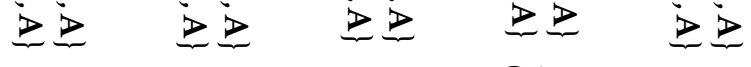

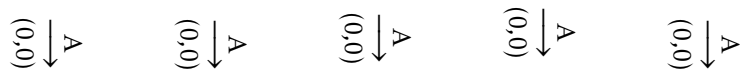

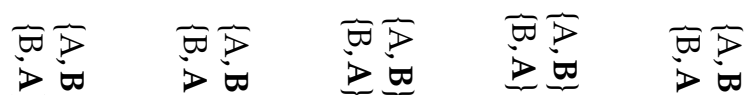

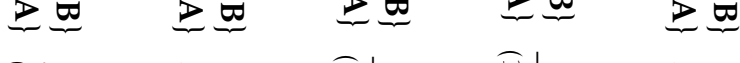

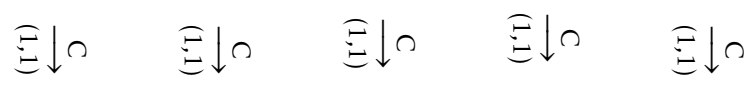

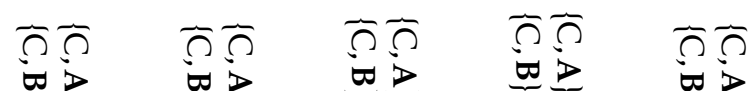

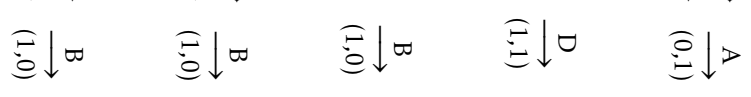

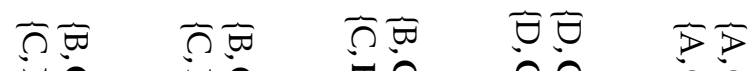

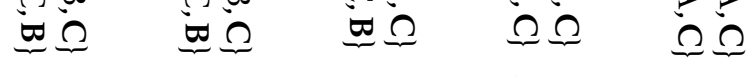

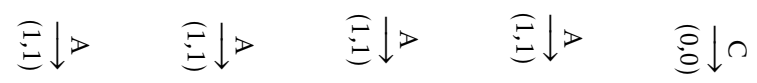

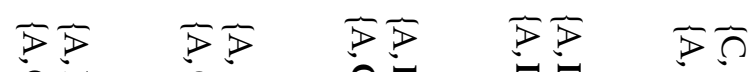

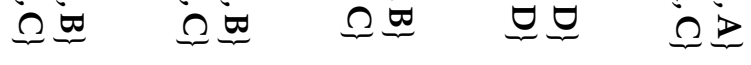

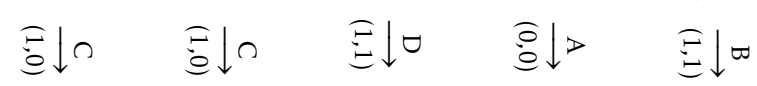

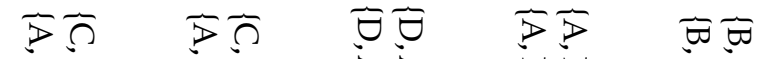

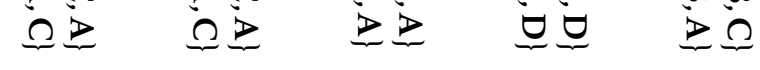

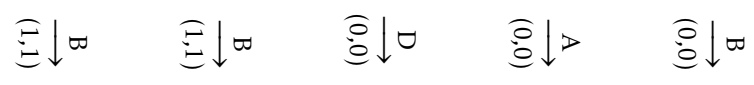

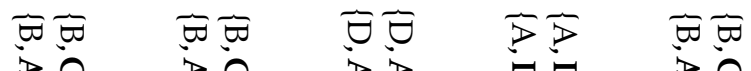

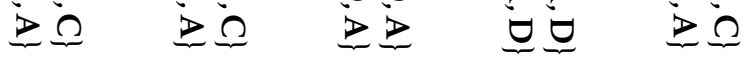

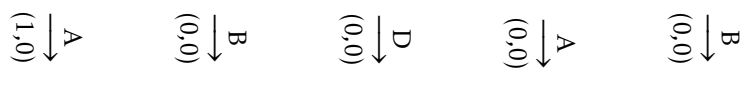

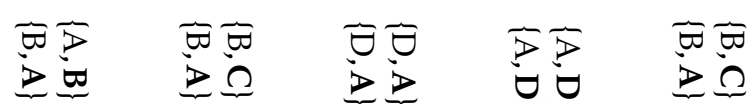

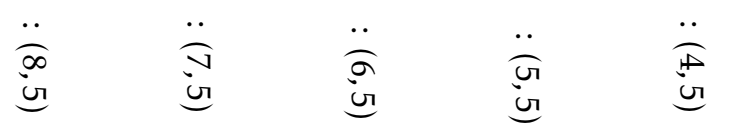

